$\begin{array}{ll}\text { environmental } & \text { sfan } \\ \text { microbiology reports } & \text { sfam }\end{array}$

\title{
Detection of human pathogenic Fusarium species in hospital and communal sink biofilms by using a highly specific monoclonal antibody
}

\begin{tabular}{|r|l|}
\hline Journal: & Environmental Microbiology and Environmental Microbiology Reports \\
\hline Manuscript ID & EMI-2015-1627.R1 \\
\hline Manuscript Type: & EMI - Research article \\
\hline Journal: & Environmental Microbiology \\
\hline Date Submitted by the Author: & 07-Jan-2016 \\
\hline Complete List of Authors: & $\begin{array}{l}\text { Al-Maqtoofi, Marwan; University of Exeter, Biosciences } \\
\text { Thornton, Christopher; University of Exeter, Biosciences }\end{array}$ \\
\hline Keywords: & $\begin{array}{l}\text { fungi, microbes and surfaces, new tools/technological developments, } \\
\text { biofilm biology }\end{array}$ \\
\hline
\end{tabular}


Fusarium-specific monoclonal antibody

1 Detection of human pathogenic Fusarium species in hospital and communal sink biofilms by using a highly specific monoclonal antibody

5 Marwan Al-Maqtoofi ${ }^{1,2}$ and Christopher R. Thornton ${ }^{1 *}$

$6 \quad$ iosciences, Geoffrey Pope Building, University of Exeter, Stocker Road, Exeter, EX4 4QD, UK.

$7 \quad{ }^{2}$ University of Basrah, College of Science, Biology Department, Basrah, Iraq.

*Corresponding author: Tel: +44 (0)1392 725172. Fax: +44 (0)1392 263434. E-mail address:

9 C.R.Thornton@ex.ac.uk

10

\section{Summary}

12 The fungus Fusarium is well known as a plant pathogen, but has recently emerged ever recent years

13 as an opportunistic pathogen of humans. Habitats providing direct human exposure to infectious

14 propagules are largely unknown, but there is growing evidence that plumbing systems are sources

15 of human pathogenic strains in the Fusarium solani species complex (FSSC) and Fusarium oxysporum species complexes(FOSC), the most common groups infecting humans. Here, we use a

17 newly developed Fusarium-specific monoclonal antibody (mAb ED7) to track FSSC and FOSC

18 strains in sink drain biofilms by detecting its target antigen, an extracellular 200kDa heat-stable carbohydrate, in saline swabs. The diagnestic -antigen was detectable in $52 \%$ of swab samples

20 collected from sinks across a University campus and a tertiary care hospital. The mAb was $100 \%$

21 accurate in detecting FSSC, FOSC and F. dimerum species complex (FDSC) strains that were

22 present, as mixed fungal communities, in $83 \%$ of sink drain biofilms. Specificity of the ELISA was 
Fusarium-specific monoclonal antibody

1

2 3 5

6

5

6

7

3

confirmed by sequencing of the internally transcribed spacer 1 (ITS1)-5.8S-ITS2 rRNA-encoding regions of culturable yeasts and moulds that were recovered using mycological culture, while translation elongation factor (TEF)-1 $\alpha$ analysis of Fusarium isolates included FSSC 1-a, FOSC 33

and FDSC ET-gr, the most common clinical pathotypes in each group. 5

\section{Originality-Significance Statement}

- Development of a monoclonal antibody (mAb ED7) specific to Fusarium, a fungal genus containing human and plant pathogens

- Fusarium diagnostic antigen detected in swabs of sink drain biofilms, with $100 \%$ accuracy

of mAb-based ELISA confirmed by ITS sequencing of mixed fungal communities 11 comprising human pathogenic yeasts and moulds

- First report describing the use of a highly specifc mAb to track human pathogenic fusaria, demonstrating widespread occurrence of pathogen in communal and hospital sinks with potential for nosocomial and community acquired infections

8

9

0

1

2


Fusarium-specific monoclonal antibody

\section{Introduction}

2 Species in the fungal genus Fusarium are ubiquitous environmental moulds, and pathogens of both

3 plants and animals (Zhang et al., 2006; Thornton and Wills, 2015). In immunocompromised

4 humans, such as patients with haematological malignancies and hematopoietic stem cell and solid

5 organ transplant recipients, Fusarium species are significant emerging pathogens, causing a

6 frequently fatal disseminated disease known as fusariosis with an associated mortality rate of 50-

7 75\% (Girmenia et al., 2000; Musa et al., 2000; Boutati and Anaissie, 1997; Dignani and Anaissie,

8 2004; Jensen et al., 2004; Nucci and Anaissie, 2007). In some tertiary cancer centres, Fusarium has

9 emerged as the second most common mould pathogen after Aspergillus (Walsh and Groll, 1999;

10 Muhammed et al., 2011). Regardless of human immune status, Fusarium species can cause

11 localised nail infections (onychomycosis)(Arrese et al., 1996), bone and joint infections (Koehler et

12 al., 2014), infections of burn wounds (Latenser, 2003), skin infections (Nucci and Anaissie, 2002;

13 Gurusidappa and Mamatha, 2011), and are the most frequent cause of mycotic eye infections known

14 as fungal keratitis (Jurkunas et al., 2009), leading to progressive corneal destruction and

15 endophthalmitis, with loss of vision or even loss of the affected eye (Dursun et al., 2003; Edelstein 16 et al., 2012).

17 A recent multistate outbreak of fungal keratitis in the USA and in Singapore and Hong Kong 18 was associated with contact lens solution contaminated with multiple strains of Fusarium and which 19 led to visual loss in many patients and the need for corneal transplantation (Chang et al., 2006).

20 While such outbreaks are rare, disseminated Fusarium infections and keratomycoses have increased

21 in frequency over the past decade (Koehler et al., 2014) and an increasing body of evidence 22 suggests that the main environmental sources of human pathogenic Fusarium species are 23 contaminated water systems (Doggett, 2000; Anaissie et al., 2002; Anaissie et al., 2011; Mehl and 
Fusarium-specific monoclonal antibody

1 Epstein, 2008). A number of studies have recovered pathogenic Fusarium species from plumbing

2 fixtures and it is hypothesised that microbial biofilms on fixtures may serve as important reservoirs

3 of infectious Fusarium propagules in hospitals and homes (Mehl and Epstein, 2008; Short et al.,

4 2011).

Identification of environmental reservoirs of human pathogenic mouldmolds including

$6 \quad$ Fusarium has typically relied on nucleic acid-based technologies following recovery of fungi using selective media (Anaissie et al., 2002; Mehl and Epstein, 2008; Short et al., 2011; Anaissie et al.,

8 2011; Rougeron et al., 2014). Recently, highly specific monoclonal antibodies (mAb) have been

9 used to identify pathogenic species or species complexes in environmental samples containing

10 mixed populations of yeasts and mouldmolds (Thornton, 2009; Davies and Thornton, 2014;

11 Thornton et al., 2015). While mAb-based approaches similarly rely on culture for recovery of fungi

12 from environmental samples, detection of diagnostic antigens in crude culture extracts using genus-

13 or species-specific mAbs offers an attractive alternative approach to pathogen detection, particularly

14 when combined with unsophisticated diagnostic modalities such as lateral-flow technology

15 (Thornton, 2008; Thornton, 2012). In this study, we set out to determine whether a newly developed Fusarium-specific mAb

17 (ED7) could be used to track the fungus by detecting a water-soluble diagnostic antigen in swabs of

18 communal and hospital sinks. By using the $\mathrm{mAb}$ in an enzyme-linked immunsorbent assay

19 (ELISA), we show that it can differentiate Fusarium species from other unrelated yeast and

20 mouldmold pathogens of humans present in mixed fungal communities encountered in sink

21 biofilms. The ELISA represents a simple method for specific detection of Fusarium species in

22 environmental reservoirs and for identifying plumbing systems contaminated with the fungus. 
Fusarium-specific monoclonal antibody

\section{Results}

2 Production of hybridoma cell lines, isotyping of $m A b$ and specificity

3 A single fusion was performed and 389 hybridoma cell lines were screened for specificity against a range of clinically relevant yeasts and molds (Table S1)mAb production. The aim was to identify cell lines secreting mAbs specific to Fusarium that could be used to track the fungus in environmental samples containing mixed species of human pathogenic fungi. To this end, aA single cell line, ED7, produced-was identified that produced mAbs belonging to the immunoglobulin class M (IgM), which was genus-specific, reacting in ELISA tests with antigens from Fusarium species

9 and with the F. solani teleomorph Haematonectria haematococca only (Figs. 1A and 1B). It did not

10 11

cross-react with antigens from a wide range of unrelated mouldmold and yeast species (Fig. 1A).

\section{Western blotting of the ED7 antigen and epitope antigen characterization-}

Gel electrophoresis and western blotting studies showed that mAb-ED7 binds to a major extracellular-antigen with molecular weight of $\sim 200 \mathrm{kDa}$ which is secreted extracellularly by both F. solani and F. oxysporum (Fig. 2A). Fusarium solani antigens were subjected to enzymatic (Fig. $\underline{2 B}$ and Fig. 2C), heat (Fig. 2D) and chemical (Fig. 2E) modifications in order to characterise the epitope bound by ED7. Reductions in mAb binding following treatment with pronase shows that its epitope consists of protein, while reductions with trypsin indicate a protein epitope containing positively charged lysine and arginine side chains. The lack of reduction in ED7 binding following digestion of immobilized antigen with trypsin (Fig. 2B) and pronase (Fig. 2C) shows that it does not bind to a protein epitope. Reductions in mAb binding following heat treatment shows that an epitope is heat labile. There was no significant reduction in ED7 binding over 70 min of heating, showing that its epitope is heat stable (Fig. 2D). Reductions in mAb binding following chemical 
Fusarium-specific monoclonal antibody

digestion of an antigen with periodate shows that its epitope is carbohydrate and contains vicinal hydroxyl groups. The pronounced reductions in ED7 binding following periodate oxidation shows that its epitope consists of carbohydrate residues (Fig. 2E). Taken together, Binding of mAb ED7 to its target antigen was unaffected by pronase (Fig. 2B) or trypsin (Fig. 2C) digestion or by heating (Fig. 2D). - tThese results, combined with significant reductions in antibody binding following 6 periodate oxidation (Fig. 2E), indicate that $\mathrm{mAb}-\mathrm{ED} 7$ binds to an extracellular antigen and that its epitope is a -heat stable carbohydrate moeityepitope containing with-vicinal hydroxyl groups.

\section{Immunofluorescence and immunogold electron microscopy}

10 Immuno-localisation studies using IF showed that the ED7 antigen was present on the surface of 11 spores and hyphae (Figs. 2F-I and 2G), while IEM showed that the antigen was present in the spore and hyphal cell wall and in an extracellular fibrillar matrix surrounding both (Figs. 2J्H-L TEM image shown in Fig. 2J, 56\% of gold particles were distributed in the fibrillar matrix surrounding the cell, while $40 \%$ and $4 \%$ of gold particles were distributed in the cell wall and within the cell wall.

17

18

19

20 $-$

Immunodetection of Fusarium species in sink swabs and identification of fungi by analysis of the ITS regions of the rRNA-encoding gene unit and by Translation Elongation Factor-1 $\alpha$ PCR

Monoclonal antibody ED7 was highly specific for the three human pathogenic species of Fusarium, F. solani, F. oxysporum and F. dimerum, which were culturable from $75 \%$ of the sink swabs (Table
Formatted: Level 1, Widow/Orphan control, Adjust space between Latin and Asian text, Adjust space between Asian text and numbers 
Fusarium-specific monoclonal antibody

$1 \quad 1$ and Table S2) 2 and summarised in Table 3). ELISA tests of the saline sink swabs showed that $52 \%$ contained detectable levels of Fusarium antigen (Table 1 and Table S2 3 ), with ELISA absorbance values in the range $\geq 0.100$ (the threshold value for antigen detection) and up to 1.500 . In four hospital samples (samples S47, S48 and S49 from ophthalmology and sample S64 from oncology) Fusarium strains could not be recovered for identification by ITS sequencing despite detection of the diagnostic antigen in swab samples with absorbance values of $0.264,0.530,0.187$ and 0.193 respectively (Table 12 ). This was likely due to the Fusarium isolates being outgrown in the mixed culture plates by faster growing or more abundant unrelated fungi. Importantly, $\mathrm{mAb}$ ED7 was shown not to cross-react with unrelated fungi (axenic culture absorbance values of $\leq 0.100$ in all cases) including the human pathogenic yeast or yeast-like fungi Candida, Exophiala, Meyerozyma, Rhodotorula, Trichosporon, the human pathogenic hyaline or dematiaceous molds Aspergillus, Phialophora, Phoma, Trichoderma, and the human pathogenic mucormycete Mucor (Table 1 z). The remaining $93 \%$ of samples positive for Fusarium antigen, either at the swab stage or following periods of biological amplification in mixed or axenic cultures (Table S2), yielded strains of the three Fusarium species. There was $100 \%$ concordance between Fusarium genus identification by ELISA and species identification by ITS sequencing (Table 13 ). The species of $F$. solani and $F$. oxypsporum recovered from sink swabs were subsequently shown by TEF-1 $\alpha$ PCR (Supporting Data Set 1) to belong to F. solani species complex (FSSC) 1-a, 1-c, 2-a, 2-v, 5-d, 5-k, 9-a, 15-a, 20-d and F. oxysporum (FOSC) species complexes 16, 33, 99, 111, 126, 134, 183 (Table S3Table 4 and Appendix 1 ). All of the recovered $F$. dimerum isolates belonged to the $F$. dimerum species complex (FDSC) ET-gr (Table S3). ITS analysis of axenic cultures (Table_1 3 ) showed that a number of sink samples (e.g. S2, S6, S17, S19, S21, S24, S30, S38) contained mixtures of 
Fusarium-specific monoclonal antibody

1 Appendix 1) showed that others contained mixtures of species complexes of the same species (e.g.

2 S8, S9, S25, S28). Monoclonal antibody ED7 was able to detect all of the Fusarium species

3 complexes recovered in this study.

4 In addition to drain swabs, water samples were collected from the taps of sinks in the

5 hospital haematology and oncology units and from the main water tanks feeding the ophthalmology

6 unit. The ED7 diagnostic antigen could not be detected in any of the water samples directly and,

7 while all of the samples yielded fungi, only two of the tap samples (oncology W57 and W60)

8 contained Fusarium strains that belonged to FDSC ET-gr. and which were detectable by ELISA at

9 the mixed culture stage (Table $\underline{12}$ ). The sink biofilms corresponding to these water samples were also positive at the swab ELISA stage (Table 1).

11

12

13

14

15

16

17

18

19

20

21

22

23 
Fusarium-specific monoclonal antibody

1

\section{Discussion}

3 The genus Fusarium comprises ubiquitous environmental mouldmolds capable of infecting plants and humans (Zhang et al., 2006). Unlike agriculture, where the most economically damaging

5 pathogens are considered to be $F$. graminearum and $F$. oxysporum (Dean et al., 2012), the species

6 most commonly cited as human pathogens belong to the Fusarium solani species complex (FSSC,

7 responsible for $50 \%$ of reported infections in humans), followed by strains in the Fusarium

8 oxysporum species complex (FOSC)(Torres and Kontoyiannis, 2011). The Fusarium dimerum

9 species complex (FDSC) is less frequently reported as causing human disease, but it is similarly

10 capable of causing disseminated infections in immunocompromised patients (Bigley et al., 2004;

11 Schroers et al., 2009).

12 While the natural habitats of plant pathogenic Fusarium strains are well characterised as soil

13 and decaying plant material, habitats providing direct human exposure to infectious propagules are

14 largely unexplored. The increasing frequency of opportunistic fungal infections in humans means

15 that improved surveillance methods are needed to identify environmental reservoirs of pathogens to

16 limit the exposure of vulnerable individuals to potentially infective propagules. For Fusarium, there

17 is a growing body of evidence to suggest that domestic and municipal water systems are potential

18 reservoirs of human pathogenic strains in the FSSC, FOSC and FDSC groups (Short et al., 2011).

19 Accurate techniques that can be used to identify the fungus in environmental samples 20 containing mixed populations of fungi are currently lacking and, while nucleic acid-based

21 technologies have been developed for the differentiation of Fusarium from other human pathogenic

22 species and to identify Fusarium species complexes, such techniques have typically been used in

23 retrospective analysis of axenic cultures collected during human and environmental population 
Fusarium-specific monoclonal antibody

studies (Bouchara et al., 2009; Steinmann et al., 2011; Lackner et al., 2012). Furthermore, these studies have often employed Fusarium-selective media that eliminate other fungi present in polymicrobial communities (Short et al., 2011). While monoclonal antibodies (mAbs) and antibody fragments have been developed for detecting and differentiating Fusarium species in vitro or in planta (Wong et al., 1988; Arie et al., 1991, 1995; Danks et al., 1996; Hayashi et al., 1998; Hu et al., 2012, 2013), no attempts have been madeused to use mAbs to track human pathogenic strains in environmental samples. Jensen et al. (2011) recently reported the development of Fusariumspecific mAbs for immunohistochemical diagnosis of fusariosis. The IgM mAbs, which recognise 51 and $63 \mathrm{kDa}$ antigens, reacted strongly with fungal elements in both experimentally infected animals and biopsy samples from patients with fusariosis sepsis and dissemination to the skin.

In this prospective study, we set out to determine whether human pathogenic species of Fusarium could be identified in sink drains directly by using crude antigen extracts of biofilms and detection using a genus-specific immunoglobulin $\mathrm{M}(\operatorname{IgM}) \mathrm{mAb}$, ED7, that binds to an $\mathrm{A}$ extracellular $\sim 200 \mathrm{kDa}$ carbohydrate antigen present on the surface of spores and hyphae. While the function of the antigen is currently unknowUsing mAb based ELISAn -we were able, in Enzyme-Linked Immunosorbent Assay (ELISA) tests, able to detect its presence the diagnostic antigen -in $52 \%$ of swab samples and, following biological amplification of biofilms on a nonselective mycological medium, were able to identify additional biofilm samples containing pathogenic strains of Fusarium. This is the first time, to our knowledge, that a mAb-based detection method has been used to track Fusarium in environmental samples. The mAb was able to differentiate Fusarium from a wide spectrum of unrelated fungi, including the human pathogens Aspergillus (Thornton and Wills, 2015), Candida, Geotrichum, Rhodotorula and Trichosporon (Davies and Thornton, 2014; Miceli et al., 2011), Cyphellophora and Phialophora (Feng et al., 
Fusarium-specific monoclonal antibody

1 2014), Exophiala (Zeng et al., 2007), Trichoderma (Sandoval-Denis et al., 2014), Engyodontium

2 (Macêdo et al., 2007; Thamke et al., 2015) and Mucor (Petrikkos et al., 2012), several of which

3 have been reported previously to inhabit biofilms in water distribution systems (Dogget, 2000). The

$4 \quad 100 \%$ accuracy of the ED7 ELISA, confirmed by using ITS sequencing and TEF PCR analysis of 11 mycological isolation procedure, means that a recognised environmental niche of this group of

recovered isolates, demonstrates its robustness in detecting potentially infectious Fusarium species in polymicrobial communities. Importantly, mAb-ED7 reacted with all of the species complex strains isolated including the most common clinical pathotypes of Fusarium, FSSC 1-a, FOSC 33 and FDSC ET-gr (Schroers et al., 2009; Short et al., 2011).

While the ED7 ELISA was able to identify Fusarium to the level of genus only, the simplicity of the mAb-based approach to detection, even when combined with a standard pathogenic fungi can be monitored readily. The widespread occurrence of human pathogenic Fusarium species in sinks of a tertiary care hospital and sinks of a heavily populated university campus, show that indoor plumbing-associated biofilms and water sources are an unseen source of Fusarium infectious propagules for nosocomial and community-acquired infections of vulnerable individuals, an observation consistent with previous studies (Annaisie et al., 2011; Short et al., 2011). While no cases of fusariosis were reported during the course of this study, tThe close proximity of the patients to hospital sinks colonised with both pathogenic fusaria and with other opportunistic fungal pathogens is a serious concern given the known vulnerability of immunocompromised individuals to invasive fungal infections. 
Fusarium-specific monoclonal antibody

1

\section{Acknowledgements}

3 M. Al-Maqtoofi was funded by a Ministry of Higher Education and Scientific Research (MOHESR)

4 of Iraq studentship (No. S634), to whom we are grateful. The authors would also like to thank the

5 RD\&E hospital for allowing us to sample patient sinks.

6

7 Conflicts of Interest

8 We declare that none of the authors involved in writing this paper have any conflicts of interest with

9 respect to the content of this article.

10

11

12

13

14

15

16

17

18

19

20

21

22

23 
Fusarium-specific monoclonal antibody

\section{Experimental procedures}

14

\section{Ethics statement}

16 All animal work described in this study was conducted under a UK Home Office Project License,

17 and was reviewed by the institution's Animal Welfare Ethical Review Board (AWERB) for

18 approval. The work was carried out in accordance with The Animals (Scientific Procedures) Act

191986 Directive 2010/63/EU, and followed all the Codes of Practice which reinforce this law,

20 including all elements of housing, care, and euthanasia of the animals. Permission for sink sampling

21 at the Royal Devon and Exeter Hospital was granted by the Director of Infection Prevention and

22 Control.

23

$24 \quad$ Fungal culture

25 Fungi (Table $\underline{S} 1)$ were routinely cultured on Potato Dextrose Agar (PDA: 70139; Sigma)),

26

27

28

29
Sabouraud Dextrose Agar (SDA: Sabouraud Dextrose Broth (SDB: S3306; Sigma) containing 2\%

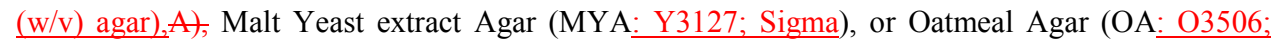

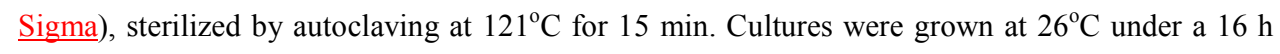

fluorescent light regime. 
Fusarium-specific monoclonal antibody

Development of $m A b$, preparation of immunogen, and immunisation regime

3 11 with $200 \mu \mathrm{l}$ of the conidial suspension and incubated with shaking $(75 \mathrm{rpm})$ for $48 \mathrm{~h}$ at $26^{\circ} \mathrm{C}$.

12 Hyphal biomass was collected on Miracloth, snap frozen in liquid $\mathrm{N}_{2}$, and lyophilized. Culture 13 filtrates were retained for gel electrophoresis and western blotting studies and stored at $-20^{\circ} \mathrm{C}$ until

$\mathrm{B} \underline{\mathrm{ALB}} \mathrm{alb} / \mathrm{c}$ mice were immunized with soluble antigens prepared from lyophilized mycelium of a human pathogenic strain of Fusarium solani species complex 1-a (CBS strain 224.34). Conidia were suspended in water after 10-day old PDA slant cultures were flooded with $5 \mathrm{ml} \mathrm{dH}_{2} \mathrm{O}$ and gently agitated with an inoculation loop. Conidial suspensions were then filtered through Miracloth to remove mycelium and transferred to $1.5 \mathrm{ml}$ micro-centrifuge tubes. The conidia were washed three times with $\mathrm{dH}_{2} \mathrm{O}$ by repeated vortexing and centrifugation at $14,462 \mathrm{~g}$ for $5 \mathrm{~min}$ and finally suspended in $\mathrm{dH}_{2} \mathrm{O}$ to give a concentration of $10^{6}$ conidia $\mathrm{ml}^{-1}$ solution. Flasks containing $100 \mathrm{ml}$ of sterilized Potato Dextrose Broth (potato dextrose broth (PDB: P6685; Sigma))-were inoculated required. One mg of lyophilized biomass was suspended in $1 \mathrm{ml}$ phosphate buffered saline (PBS: $\left.0.8 \% \mathrm{NaCl} ; 0.02 \% \mathrm{KCl} ; 0.115 \% \mathrm{Na}_{2} \mathrm{HPO}_{4} ; 0.02 \% \mathrm{KH}_{2} \mathrm{PO}_{4} ; \mathrm{pH} 7.2\right)$ and the resultant suspension centrifuged for $5 \mathrm{~min}$ at $14,462 \mathrm{~g}$. The supernatant, containing solubilized antigens, was used as the immunogen and as a source of antigens for hybridoma screening assays. For immunization, 6-wkold $\mathrm{BALB} / \mathrm{c}$ female white mice were given four intraperitoneal injections (300 $\mu$ l per injection) of antigen extract containing $2.3 \mathrm{mg}$ protein $\mathrm{ml}^{-1}$ PBS at 2-wk intervals and a single booster injection five days before fusion.

1

Production and screening of hybridomas and determination of antibody specificity 
Fusarium-specific monoclonal antibody

1 Hybridoma cells were produced by the method described elsewhere (Thornton, 2001) and the

2 16 IgA, and IgM) peroxidase conjugate (A-0412; Sigma Chemical Company, Poole, United Kingdom)

17 diluted 1 in 1000 in PBST for a further hour. Bound antibody was visualized by incubating wells rinse with PBS before addition of the substrate solution. - -Working volumes were $50 \mu \mathrm{l}$ per well;

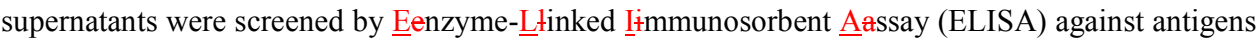
immobilized to the wells of Maxisorp microtitre plates (442404; Nunc)(50 $\mu \mathrm{l}$ per well). For antibody specificity tests, fungi were grown on replicate agar slopes and surface washings containing water-soluble antigens prepared as described in Thornton (2001). Protein concentrations, determined spectrophotometrically at $280 \mathrm{~nm}$ (Nanodrop, Agilent Technologies Limited, Berkshire, UK), were adjusted to $60 \mu \mathrm{g} \mathrm{ml}^{-1}$ buffer. Fifty $\mu \mathrm{l}$ volumes were then used to coat the wells of microtitre plates. After incubating overnight at $4^{\circ} \mathrm{C}$, wells were washed four times with PBST (PBS containing Tween-20, $0.05 \%(\mathrm{v} / \mathrm{v}))_{2}$ and_once each with PBS and $\mathrm{dH}_{2} \mathrm{O}$ and then air-dried at $23^{\circ} \mathrm{C}$ in a laminar flow hood. The plates were stored in sealed plastic bags at $4^{\circ} \mathrm{C}$ in preparation for screening of hybridoma supernatants by ELISA as described below.

\section{Plate-Trapped-Antigen-Enzyme-Linked Immunosorbent Assay}

Wells containing immobilized antigens were incubated successively with hybridoma tissue culture supernatant (TCS) for $1 \mathrm{~h}$, followed with goat anti-mouse polyvalent (immunoglobulin classes IgG, with tetramethyl benzidine (TMB: T-2885; Sigma) substrate solution (Thornton, 2001) for 30 min. The reactions were stopped by the addition of $3 \mathrm{M} \mathrm{H}_{2} \mathrm{SO}_{4}$ and - $\underline{\mathrm{a}} \mathrm{Absorbance}$ values were determined at $450 \mathrm{~nm}$ with an MRX automated microplate reader (Dynex Technologies, Billingshurst, UK). Wells were given four 5-min rinses with PBST between incubations and a final and control wells were incubated with tissue culture medium (TCM) containing $10 \%(\mathrm{v} / \mathrm{v})$ fetal 
Fusarium-specific monoclonal antibody

1 bovine serum. All incubation steps were performed at $23^{\circ} \mathrm{C}$ in sealed plastic bags. The threshold for

2 detection of the antigen in ELISA was determined from control means $(2 \mathrm{x}$ TCM absorbance

3 values)(Sutula et al., 1986). These values were consistently in the range $0.050-0.100$. Consequently,

4 absorbance values $>0.100$ were considered as positive for the detection of antigen.

6 Determination of Ig subclass and cloning procedure

7 The Ig class of mAbs was determined by using antigen-mediated ELISA. Wells of microtitre plates

coated with $F$. solani CBS224.34 water-soluble antigens from surface washings were incubated successively with hybridoma supernatant ED7 TCS for $1 \mathrm{~h}$, followed with goat anti-mouse $\mathrm{IgG}_{1}$, $\operatorname{IgG}_{2 \mathrm{a}}, \operatorname{IgG}_{2 \mathrm{~b}}, \operatorname{IgG}_{3}$, IgM, or IgA-specific antiserum (ISO-2; Sigma) diluted 1 in 3000 in PBST for 30 min and rabbit anti-goat peroxidase conjugate diluted 1 in 1000 (A-5420; Sigma) for a further 30 min. Bound antibody was visualized with TMB substrate as described above. Hybridoma cells lines were sub-cloned three times by limiting dilution, and cell lines were grown in bulk in a nonselective medium preserved by slowly freezing in fetal bovine serum/dimethyl sulfoxide (92:8 $[\mathrm{v} / \mathrm{v}])$, and stored in liquid nitrogen.

\section{Gel electrophoresis and Western blotting}

For sodium-dodecyl-sulphate-polyacrylamide gel electrophoresis (SDS-PAGE), culture filtrates from 2-d-old PDB shake cultures of $F$. solani CBS224.34 and F. oxysporum f.sp. lycopersici CBS167.30,-prepared as described, were diluted in Laemmli buffer (Laemmli, 1970) and were denatured by heating at $95^{\circ} \mathrm{C}$ for $10 \mathrm{~min}$. Antigens were separated in $4-20 \%(\mathrm{w} / \mathrm{v})$ polyacrylamide gradient gels (161-1159; Bio-Rad) for $1.5 \mathrm{~h}$ at $23^{\circ} \mathrm{C}(165 \mathrm{~V})$ under denaturing conditions, and prestained broad range markers (161-0318; Bio-Rad) were used for molecular weight determinations. 
Fusarium-specific monoclonal antibody

1

\section{Characterization of antigen by enzymatic and chemical modifications and by heating}

For $\underline{w} W e s t e r n s$, separated antigens were transferred electrophoretically to a PVDF membrane (1620175; Bio-Rad). The membranes were blocked for $16 \mathrm{~h}$ at $4^{\circ} \mathrm{C}$ with $\mathrm{PBS}$ containing $1 \%(\mathrm{w} / \mathrm{v})$ bovine serum albumin (BSA) and incubated with hybridema supernatantED7 TCS diluted 1 in 2 with PBS containing $0.5 \%(\mathrm{w} / \mathrm{v}) \mathrm{BSA}(\mathrm{PBSA})$ for $2 \mathrm{~h}$ at $23^{\circ} \mathrm{C}$. After washing three times with PBS, membranes were incubated for $1 \mathrm{~h}$ with goat anti-mouse $\operatorname{IgM}$ ( $\mu$-chain specific) alkaline phosphatase conjugate (A-9688; Sigma), diluted 1 in 15,000 in PBSA. After the membranes were washed twice with PBS and once with PBST, the bound antibodies were visualized by incubation in $\underline{\mathrm{BCIP} / \mathrm{NBT}}$ substrate solution. Reactions were stopped by immersion in $\mathrm{dH}_{2} \mathrm{O}$ and air-dried between sheets of Whatman filter paper.

Water-soluble antigens from surface washings of slopes of $F$. solani CBS224.34 were prepared as described. Heat stability studies were conducted by placing tubes of solubilised antigen solubilised antigen from three replicate cultures of $F$. solani CBS224.34-in a boiling water bath. At 10 min intervals, samples were removed, centrifuged at 14,462 g 14,500 rpm-for $5 \mathrm{~min}$, and antigens immobilised to the wells of microtitre plates for assay by ELISA as described. For periodate oxidation, microtitre wells containing immobilised antigens from surface washings of the fungusantigens were incubated with $50 \mu \mathrm{l}$ of sodium meta-periodate solution $\left(20 \mathrm{mM} \mathrm{NaIO}_{4}\right.$ in 50 $\mathrm{mM}$ sodium acetate buffer ( $\mathrm{pH} 4.5)$ ) or acetate buffer only (control) at $4^{\circ} \mathrm{C}$ in sealed plastic bags.

Plates were given four 3-min PBS washes before processing by ELISA as described. For protease digestions, microtitre wells containing immobilised antigen were incubated with $50 \mu$ of pronase (protease XIV; $9 \mathrm{mg} \mathrm{ml}^{-1}$ in PBS) or trypsin $\left(1 \mathrm{mg} \mathrm{ml}^{-1}\right.$ in Milli-Q $\left.\mathrm{H}_{2} \mathrm{O}\right)$ solution or Milli-Q $\mathrm{H}_{2} \mathrm{O}$ or 3 PBS only controls respectively for $4 \mathrm{~h}$ at $37^{\circ} \mathrm{C}$ or $4^{\circ} \mathrm{C}$. Plates were given four 3-min rinses with 
Fusarium-specific monoclonal antibody

1

3

4 For immunfluorescence (IF), sterilised slides were coated with a washed spore suspensions of $F$.

PBS and then assayed by ELISA with hybridoma supernatantED7 TCS as described.

\section{Immunofluorescence and immunogold electron microscopy} solani CBS224.34 containing $1 \% \underline{(\mathrm{w} / \mathrm{v})}$ glucose solution and incubated at $26^{\circ} \mathrm{C}$ for $16 \mathrm{~h}$ to allow spore germination and formation of germ tubes. After air-drying, the stides-cells were fixed to the slides as described in Thornton (2001) and incubated with hybridema supernatantED7 TCS or TCM only (negative control) for $1 \mathrm{~h}$, followed by three $5 \mathrm{~min}$ PBS washes. Slides were then incubated with goat anti-mouse polyvalent fluorescein isothiocyanate (FITC) conjugate (diluted 1 in 40 in PBS)(F1010; Sigma) for $30 \mathrm{~min}$. Slides were given three 5 min washes with PBS and mounted in PBS-glycerol mounting medium (F4680; Sigma) before overlaying with coverslips. All incubation steps were performed at $23^{\circ} \mathrm{C}$ in a humid environment to prevent evaporation and slides were stored in the dark, at $4^{\circ} \mathrm{C}$, prior to examination using an epifluorescence microscope (Olympus IX81) fitted with $495 \mathrm{~nm}$ (excitation) and $518 \mathrm{~nm}$ (emission) filters for FITC. For immunogold electron microscopy (IEM)_, the method spores were embedded in LR White resin and immunostained by using hybridoma supernatant or TCM control and anti-mouse polyvalent $20 \mathrm{~nm}$ gold conjugate according to the technique described in Thornton \& Talbot (2001) was used. Spores and hyphae of F. solani were prepared by incubating washed conidia in $1 \%(\mathrm{w} / \mathrm{v})$ glucose solution at $26^{\circ} \mathrm{C}$ for 16 $\underline{\mathrm{h} \text { to allow spore germination and formation of germ tubes. Cells were embedded in LR White resin }}$ (Agar Scientific Ltd.) and ultra thin sections prepared for immunolabeling. Sections immobilized to $\underline{\text { nickel grids were blocked by immersion in PBST containing } 1 \%(\mathrm{w} / \mathrm{v}) \text { BSA (PBST-BSA) which }}$ had been sterile filtered through a $0.2 \mu \mathrm{m}$ filter. The grids were washed three times $(3 \mathrm{~min}$ each) in $\underline{\text { sterile filtered PBST and then incubated in ED7 TCS or TCM only (negative control) for } 1 \mathrm{~h} \text {. After }}$
Formatted: Font: Italic

Formatted: Font: Symbol 
Fusarium-specific monoclonal antibody

four washes (3 min each) with sterile filtered PBST, the grids were incubated for a further hour in

$\underline{\text { PBST-BSA containing a 1:20 dilution of goat anti-mouse } 20 \mathrm{~nm} \text { gold conjugate (EM.GAF20; BBI }}$

$\underline{\text { Solutions). The grids were washed four times ( } 3 \mathrm{~min} \text { each) in sterile filtered PBST and then placed }}$

on Whatman filter paper to dry. Dried grids were then incubated for $20 \mathrm{~min}$ in $2 \%(\mathrm{w} / \mathrm{v})$ uranyl

acetate solution followed by $2 \%(\mathrm{w} / \mathrm{v})$ lead citrate solution for $4 \mathrm{~min}$. Working volumes were $100 \mu \mathrm{l}$

Formatted: Font: Symbol

and incubation and washing steps were carried out at at $23^{\circ} \mathrm{C}$. Immunostained samples were

examined using a Jeol JEM 1400 transmission electron microscope fitted with a Gatan ES 100W

8 CCD camera.

9

10 Statistical analysis

11 Unless otherwise stated, numerical data were analysed using the statistical programme Minitab

12 (Minitab 16, Minitab ${ }^{\circledR}$, Coventry, UK). Analysis of variance (ANOVA) was used to compare

13 means of more than two data sets and Post-hoc Tukey-Kramer analysis was then performed to

14 distinguish which sets were significantly different from one another.

15

16 Sampling from drains

17 A total of 65 sinks were swabbed, comprising 32 sinks across the ICU, ITU, haematology, oncology

18 and ophthalmology units of the Royal Devon and Exeter tertiary care hospital (Exeter, Devon, UK)

19 and 33 restroom sinks located around the University of Exeter campus (Exeter, Devon, UK). In

20 addition, cold-water samples were collected from taps connected to the sinks in the haematology

21 and oncology unit, and from the two main water tanks feeding the ophthalmology unit. To isolate

22 fungi from sink biofilms, sterile cotton buds (Boots, UK) wetted with PBS were used to scour the

23 inner surfaces of sink drainpipes for approximately $10 \mathrm{~s}$. Swabs with visible detritus were immersed 
Fusarium-specific monoclonal antibody

1 in $1.5-\mathrm{ml}$ micro-centrifuge tubes containing $1 \mathrm{ml}$ PBS to dislodge biofilm debris, and the sealed

2 tubes transferred to the laboratory for processing by ELISA and mycological culture.

Immunodetection of Fusarium species in sink swabs and identification of fungi by analysis of the

ITS regions of the rRNA-encoding gene unit and Translation Elongation Factor-1 $\alpha$ PCR

Biofilm debris was pelleted by centrifugation at $14,462 \mathrm{~g}$ for $5 \mathrm{~min}$ and $50 \mu \mathrm{l}$ samples of

supernatant transferred to the wells of microtitre plates for assay by ELISA (Table 12; SwabELISAand Table S2; Swab-ELISA) as described. The biofilm pellet was re-suspended in $1 \mathrm{ml}$ $\mathrm{dH}_{2} \mathrm{O}, 200 \mu \mathrm{l}$ samples spread on the surface of PDA containing the $1 \mu \mathrm{g} \mathrm{ml}^{-1}$ of the broad-spectrum antibiotic rifampicin, and the plates incubated for $2 \mathrm{~d}$ at $26^{\circ} \mathrm{C}$ under a $16 \mathrm{~h}$ fluorescent light regime.

11 Fungi in these mixed culture plates were separated on the basis of gross morphological

characteristics and axenic slope cultures generated following sub-culture on PDA. Crude antigen extracts were prepared as surface washings from mixed cultures and from axenic cultures and

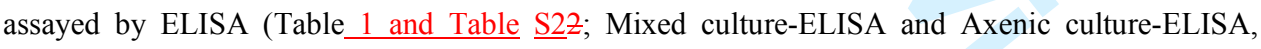
respectively) as described.

Fungal DNA was extracted from axenic culture material by using the CTAB method (Chow \& Kafer, 1993) and fungi were identified by sequencing of the ITS1-5.8S-ITS2 region of the rRNAencoding gene unit (White et al., 1990) according to procedures described elsewhere (Thornton et al., 2002), using the primers ITS1ext (5'-GTAACAAGGTTTCCGTAGGTG-3') and ITS4ext (5'-

TTCTTTTCCTCCGCTTATTGATATGC-3'). Species identity was predicted based on $>95 \%$ sequence identity $(E-v a l u e=0.0)($ Altschul et al. 1997) of the ITS1-5.8S-ITS2 region of recovered species to species recorded in GenBank. Fusarium species were further identified to species complex level by using the forward primer ef-1 (5'-ATGGGTAAGGA(A/G)GACAAGAC-3') and 
Fusarium-specific monoclonal antibody

reverse primer ef-2 (5'-GGA(G/A)GTACCAGT(G/C)ATCATGTT-3'), which amplify an $\sim 700$ bp

region of Translation Elongation Factor 1-alpha (TEF-1 $\alpha$ ), the single-locus identification tool in

Fusarium (Geiser et al., 2004). PCR reactions were carried out in a total volume of $25 \mu$ consisting of $1 \mu \mathrm{l}$ DNA at a concentration of $30-75 \mathrm{ng} \mu \mathrm{l}^{-1}, 12.5 \mu \mathrm{l}$ of GoTaq ${ }^{\circledR}$ Green Master Mix DNA polymerase (Promega, MF7112), $9.5 \mu \mathrm{l}$ of nuclease free water (Promega) and $1 \mu \mathrm{l}$ of each primer at

6 | 20 pmol. The following cycling parameters were used: an initial denaturation step at $95-{ }^{\circ} \mathrm{C}$ for 8 min; 35 cycles of $15 \mathrm{sec}$ at $95-{ }^{\circ} \mathrm{C}$ (denaturation); $20 \mathrm{~s}$ at $54-{ }^{\circ} \mathrm{C}$ (annealing), $1 \mathrm{~min}$ at $72-{ }^{\circ} \mathrm{C}$ (extension) followed by a final $5 \mathrm{~min}$ extension step at $72{ }^{\circ} \mathrm{C}$. Phylogenetic sub-groups of Fusarium

9 species were determined by interrogation of the FUSARIUM-ID v. 1.0 database

10 (http://isolate.fusariumdb.org)(O’Donnell et al., 2010), with the newly acquired TEF-1 $\alpha$ sequences

11 (Supporting Data Set 1Appendix 1).

12

13 Nucleotide sequence accession numbers

14 Newly determined ITS sequences were submitted to GenBank and the ITS accession numbers

15 KT876496 to KT876723 were obtained. Species designations of recovered fungi are shown in Table

$16 \mid \underline{1} z$.

17

18

19

20

21

22

23 
Fusarium-specific monoclonal antibody

3

4

5

6

7

8

9

10

11

12

13

14 15

\section{References}

Altschul, S.F., Madden, T.L., Schaffer, A.A., Zhang, Z., Miller, W., and Lipman, D.J. (1997) Gapped BLAST and PSI-BLAST: a new generation of protein database programs. Nucleic Acids Res 25: 3389-3402.

Anaissie, E.J., Kuchar, R.T., Rex, J.H., Francesconi, A., Kasai, M., Muller, F.M., et al. (2011) Fusariosis associated with pathogenic Fusarium species colonization of a hospital water system: a new paradigm for the epidemiology of opportunistic mouldmold pathogens. Clin Infect Dis 33: 1871-1878.

Anaissie, E.J., Penzak, S.R., and Dignani, M.C. (2002) The hospital water supply as a source of nosocomial infections: a plea for action. Arch Intern Med 162: 1483-1492.

Arie, T., Hayashi, Y., Nagatani, A., Furuya, M., and Yamaguchi, I. (1991) Production and partial characterization of monoclonal antibodies against Fusarium oxysporum 860926a. Ann Phytopathol Soc Jpn 57: 696-701.

Arie, T., Hayashi, Y., Yoneyama K, Nagatani, A., Furuya, M., and Yamaguchi, I. (1995) Detection of Fusarium spp. in plants with monoclonal antibody. Ann Phytopathol Soc Jpn 61: 311-317. 
Fusarium-specific monoclonal antibody

Arrese, J.E., Piérard-Franchimont, C., and Piérard, G.E. (1996) Fatal hyalohyphomycosis following Fusarium onychomycosis in an immunocompromised patient. Am J Dermatopathol 18: 196198.

Bigley, V.H., Duarte, R.F., Gosling, R.D., Kibbler, C.C., Seaton, S., and Potter, M. (2004) Fusarium dimerum infection in a stem cell transplant recipient treated successfully with voriconazole. Bone Marrow Transplant 34: 815-817.

Bouchara, J-P., Hsieh, H.Y., Croquefer, S., Barton, R., Marchais, V., Pihet, M., et al. (2009) Development of an oligonucleotide array for direct detection of fungi in sputum samples from patients with cystic fibrosis. J Clin Microbiol 47: 142-152.

Boutati, E.I., and Anaissie, E.J. (1997) Fusarium, a significant emerging pathogen in patients with

Formatted: Font: Times, $12 \mathrm{pt}$ Formatted: Font: Times, 12 pt, Italic Formatted: Font: Times, $12 \mathrm{pt}$ Formatted: Font: Times, 12 pt, Italic Formatted: Font: Times, $12 \mathrm{pt}$ 
Fusarium-specific monoclonal antibody

Dignani, M.C., and Anaissie, E. (2004) Human fusariosis. Clin Microbiol Infect 10: 67-75.

Doggett, M.S. (2000) Characterisation of fungal biofilms within a municipal water distribution system. Appl Env Microbiol 66: 1249-1251.

Dursun, D., Fernandez, V., and Miller, D. (2003) Advanced Fusarium keratitis progressing to endophthalmitis. Cornea 22: 300-303.

Edelstein, S.L., Akduman, L., Durham, B.H., Fothergill, A.W., and Hsu, H.Y. (2012) Resistant Fusarium keratitis progressing to endophthalmitis. Ophthalmology Contact Lens 38: 331-335.

Feng, P., Qiaoyun, L., Najafzadeh, M.J., Gerrits van den Ende, A.H.G., Sun, J., Li, R., et al. (2014) Cyphellophora and its relatives in Phialophora: biodiversity and possible role in human disease. Fungal Diversity 65: 17-45.

Geiser, D.M., del Mar Jiménez-Gasco, M., Kang, S., Makalowska, I., Veeraraghavan, N., Ward, T.D., et al. (2004) FUSARIUM-ID v.1.0: A DNA sequence database for identifying Fusarium. Eur J Pl Pathol 110: 473-479.

Girmenia, C., Pagano, L., Corvatta, L., Mele, L., Del, A., Favero, P., et al. (2000) The epidemiology of fusarioses in patients with haematological diseases. Br J Haematol 111: 272276.

Gurusidappa, S.B., and Mamatha, H.S. (2011) Fusarial skin lesion in immunocompromised. Ind $J$ Cancer 48: 116-117.

Hayashi, Y., Arie, T., Yoneyama, K., and Yamaguchi, I. (1998) Characterisation of the antigenic determinant on Fusarium oxysporum recognized by a genus-specific monoclonal antibody. $J$ Gen Appl Microbiol 44: 43-47. 
Fusarium-specific monoclonal antibody

Hu, Z-Q., Liu, J-L., Li, H-P., Xing, S., Xue, S., Zhang, J-B., et al. (2012) Generation of a highly reactive chicken-derived single-chain variable fragment against Fusarium verticillioides by phage display. Int J Mol Sci 13: 7038-7056.

Hu, Z-Q., Li, H-P., Zhang, J-B., Huang, T., Liu, J-L., Xue, S., et al. (2013) A phage-displayed chicken single-chain antibody fused to alkaline phosphatase detects Fusarium pathogens and their presence in cereal grains. Anal Chim Acta 764: 84-92.

Jensen, T.G., Gahrn-Hansen, B., Arendrup, M., and Bruun, B. (2004) Fusarium fungaemia in immunocompromised patients. Clin Microbiol Infect 10: 499-501.

Jensen, H.M.E., Aalbæk, B., Jungersen, G., Hartvig, T., Moser, C., Rozell, B.L., et al. (2011) Immunohistochemical diagnosis of fusariosis with monoclonal antibodies. Mycoses 54: s54s55.

Jurkunas, U., Behlau, I., and Colby, K. (2009) Fungal keratitis: changing pathogens and risk factors. Cornea 28: 638-643.

Koehler, P., Tacke, D., and Cornely, O.A. (2014) Bone and joint infections by Mucorales, Scedosporium, Fusarium and even rarer fungi. Crit Rev Microbiol. doi: 10.3109/1040841X.2014.910749

Lackner, M., Najafzadeh, M.J., Sun, J., Lu, Q., and de Hoog, G.S. (2012) Rapid identification of Pseudallescheria and Scedosporium strains by using rolling circle amplification. Appl Environ Microbiol 78: 126-133.

Latenser, B.A. (2003) Fusarium infections in burn patients: a case report and review of the literature. J Burn Care Rehabil 24: 285-288.

Formatted: Font: Times, $12 \mathrm{pt}$ Formatted: Font: Times, $12 \mathrm{pt}$ Formatted: Font: Times, $12 \mathrm{pt}$, Italic Formatted: Font: Times, $12 \mathrm{pt}$ Formatted: Font: Times, 12 pt, Italic Formatted: Font: Times, $12 \mathrm{pt}$ Formatted: Font: Times, $12 \mathrm{pt}$, Italic Formatted: Font: Times, $12 \mathrm{pt}$ 
Fusarium-specific monoclonal antibody

Mehl, H.L., and Epstein, L. (2008) Sewage and community shower drains are environmental reservoirs of Fusarium species complex group 1, a human and plant pathogen. Env Microbiol 10: 219-227.

Miceli, M.H., Díaz, J.A., and Lee, S.A. (2011) Emerging opportunistic yeast infections. Lancet Infect Dis 11: 142-151.

Muhammed, M., Carneiro, H., Coleman, J., and Mylonakis, E. (2011) The challenge of managing fusariosis. Virulence 2: 91-96.

Musa, H.O., Al Eisa, A., Halim, M., Sahovic, E., Gyger, M., Chaudhri, N., et al. (2000) The spectrum of fusarium infection in immunocompromised patients with haematological malignancies and in non-immunocompromised patients; A single institution experience over 10 years. Br J Haematol 108: 544-548.

Nucci, M., and Anaissie, E. (2002) Cutaneous infection by Fusarium species in healthy and immunocompromised hosts: implications for diagnosis and management. Clin Infect Dis 35: 909-920.

Nucci, N., and Anaissie, E. (2007) Fusarium infections in immuncompromised patients. Clin Microbiol Rev 20: 695-704.

O’Donnell, K., Sutton, D.A., Rinaldi, M.G., Sarver, B.A.J., Arunmozhi Balajee, S., Schroers, H-J., et al. (2010) Internet-accessible DNA sequence database for identifying Fusaria from human and animal infections. J Clin Microbiol 48: 3708-3718.

Petrikkos, G., Skiada, A., Lortholary, O., Roilides, E., Walsh, T.J., and Kontoyiannis, D.P. (2012) Epidemiology and clinical manifestations of mucormycosis. Clin Infect Dis 54: S23-S24. 
Fusarium-specific monoclonal antibody

Rougeron, A., Schuliar, G., Leto, J., Sitterle, E., Landry, D., Bougnoux, M.E., et al. (2014) Humanimpacted areas of France are environmental reservoirs of the Pseudallescheria boydii/Scedosporium apiospermum species complex. Env Microbiol 17: 1039-1048.

Sandoval-Denis, M., Sutton, D.A., Cano-Lira, J.F., Gene, J., Fothergill, A.W., Wiederhold, N.P., et al. (2014) Phylogeny of the clinically relevant species of the emerging fungus Trichoderma and their antifungal susceptibilities. J Clin Microbiol 52: 2112-2125.

Schroers, H.-J., O’Donnell, K., Lamprecht, S.C., Kammophthalmologyr, P.L., Johnson, S., Sutton, D.A., et al. (2009) Taxonomy and phylogeny of the Fusarium dimerum species group. Mycologia 101: 44-70.

Short, D.P.G., O’Donnell, K., Zhang, N., Juba, J.H., and Geiser, D.M. (2011) Widespread occurrence of diverse human pathogenic types of the fungus Fusarium detected in plumbing drains. J Clin Microbiol 49: 4264-4272.

Steinmann, J., Schmidt, D., Buer, J., and Rath, P-M. (2011) Discrimination of Scedosporium prolificans against Pseudallescheria boydii and Scedosporium apiospermum by semiautomated repetitive sequence-based PCR. Med Mycol 49: 475-483.

Sutula, C.L., Gillett, J.M., Morrisey, S.M., and Ramsdell, D.C. (1986) Interpreting ELISA data and establishing the positive-negative threshold. Plant Dis 70: 722-26.

Thamke, D.C., Mediratta, D.K., Dhabarde, A., and Shukla, A.K. (2015) Mycotic keratitis due to Engyodontium album: first case report from India. Indian J Med Microbiol 33: 303-304.

Formatted: Font: Times, $12 \mathrm{pt}$ Formatted: Font: Times, $12 \mathrm{pt}$, Italic Formatted: Font: Times, $12 \mathrm{pt}$ Formatted: Font: Times, 12 pt, Italic Formatted: Font: Times, $12 \mathrm{pt}$ Formatted: Font: Times, $12 \mathrm{pt}$, Italic Formatted: Font: Times, $12 \mathrm{pt}$ Formatted: Font: Times, $12 \mathrm{pt}$, Italic Formatted: Font: Times, $12 \mathrm{pt}$ Formatted: Font: Times, $12 \mathrm{pt}$, Italic Formatted: Font: Times, 12 pt 
Fusarium-specific monoclonal antibody

1 Thornton, C.R. (2008) Development of an immunochromatographic lateral flow device for rapid serodiagnosis of invasive aspergillosis. Clin Vacc Immunol 15: 1095-1105.

3 Thornton, C.R. (2009) Tracking the emerging human pathogen Pseudallescheria boydii by using highly specific monoclonal antibodies. Clin Vacc Immunol 16: 756-64.

Thornton, C.R. (2012) Serological techniques for diagnosis. In Fungal Plant Pathogens, Principles and Protocols. Lane, C., Beales, P., Hughes, K. (eds.). Wallingford, UK: CABI, pp. 159-177.

Thornton, C.R., and Wills, O.E. (2015) Immunodetection of fungal and oomycete pathogens: Established and emerging threats to human health, animal welfare, and global food security. Crit Rev Microbiol. 41: 27-51.

Thornton, C.R., Ryder, L.S., Le Cocq, K., and Soanes, D.M. (2015) Identifying the emerging human pathogen Scedosporium prolificans by using a species-specific monoclonal antibody that binds to the melanin biosynthetic enzyme tetrahydroxynaphthalene reductase. Env Microbiol 17: 1023-1048.

Torres, H.J., and Kontoyiannis, D. (2011) Hyalohyphomycosis (hyaline mouldmolds). In Essentials of Clinical Mycology, Kauffman, C.A., Pappas, P.G., Sobel, J.D., and Dismukes, W.E. (eds.). New York, USA: Springer Science+Business Media, pp. 281-304.

Walsh, T.J., and Groll, A.H. (1999) Emerging fungal pathogens: evolving challenges to immunocompromised patients for the twenty-first century. Transpl Infect Dis 1:247-261.

Wong, W.C., White, M., and Wright, I.G. (1988) Production of monoclonal antibodies to Fusarium oxysporum f.sp. cubense race 4. Lett Appl Microbiol 6: 39-42.

21 Zeng, J.S., Sutton, D.A., Fothergill, A.W., Rinaldi, M.G., Harrak, M.J., and de Hoog, G.S. (2007) $3713-3720$. 
Fusarium-specific monoclonal antibody

1 Zhang, N., O’Donnell, K., Sutton, D.A., Nalim, F.A., Summerbell, R.C., Padhye, A.A., et al. (2006)

2 Members of the Fusarium solani species complex that cause infections in both humans and 3 plants are common in the environment. J Clin Microbiol 44: 2186-2190.

4

5

6

7

8

9

10

11

12

13

14

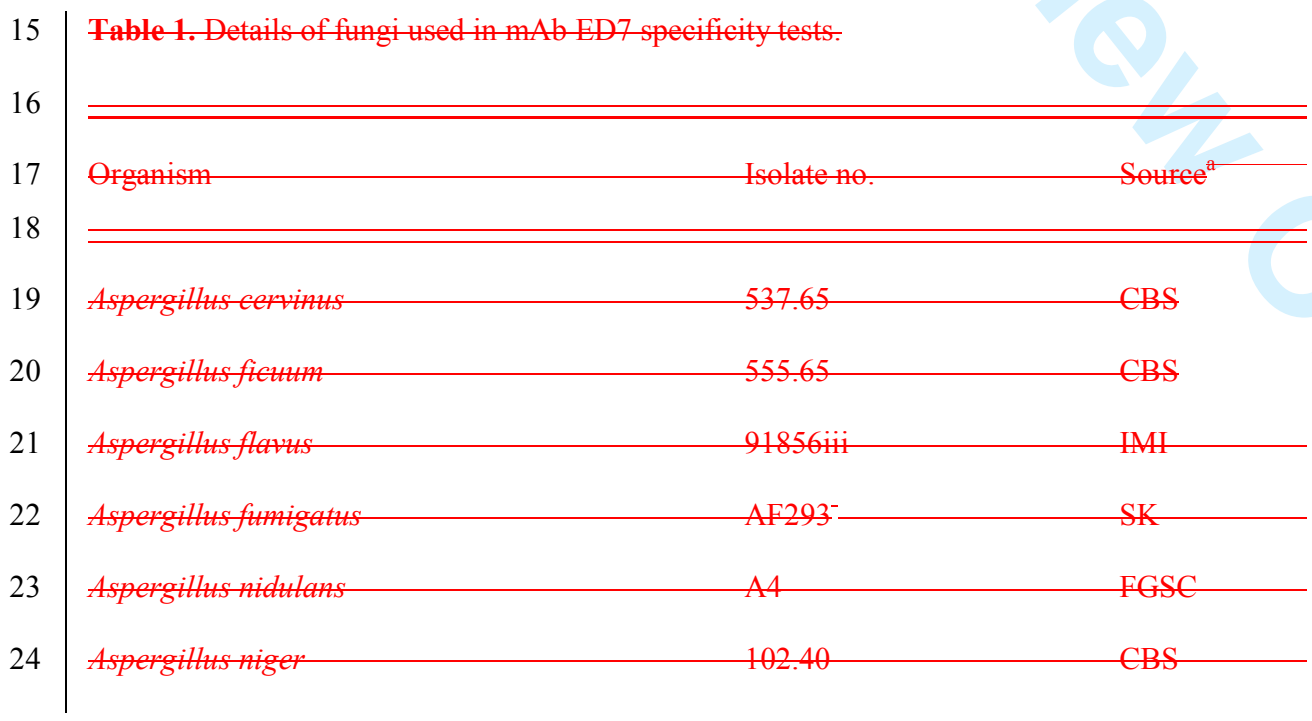

29 
Fusarium-specific monoclonal antibody

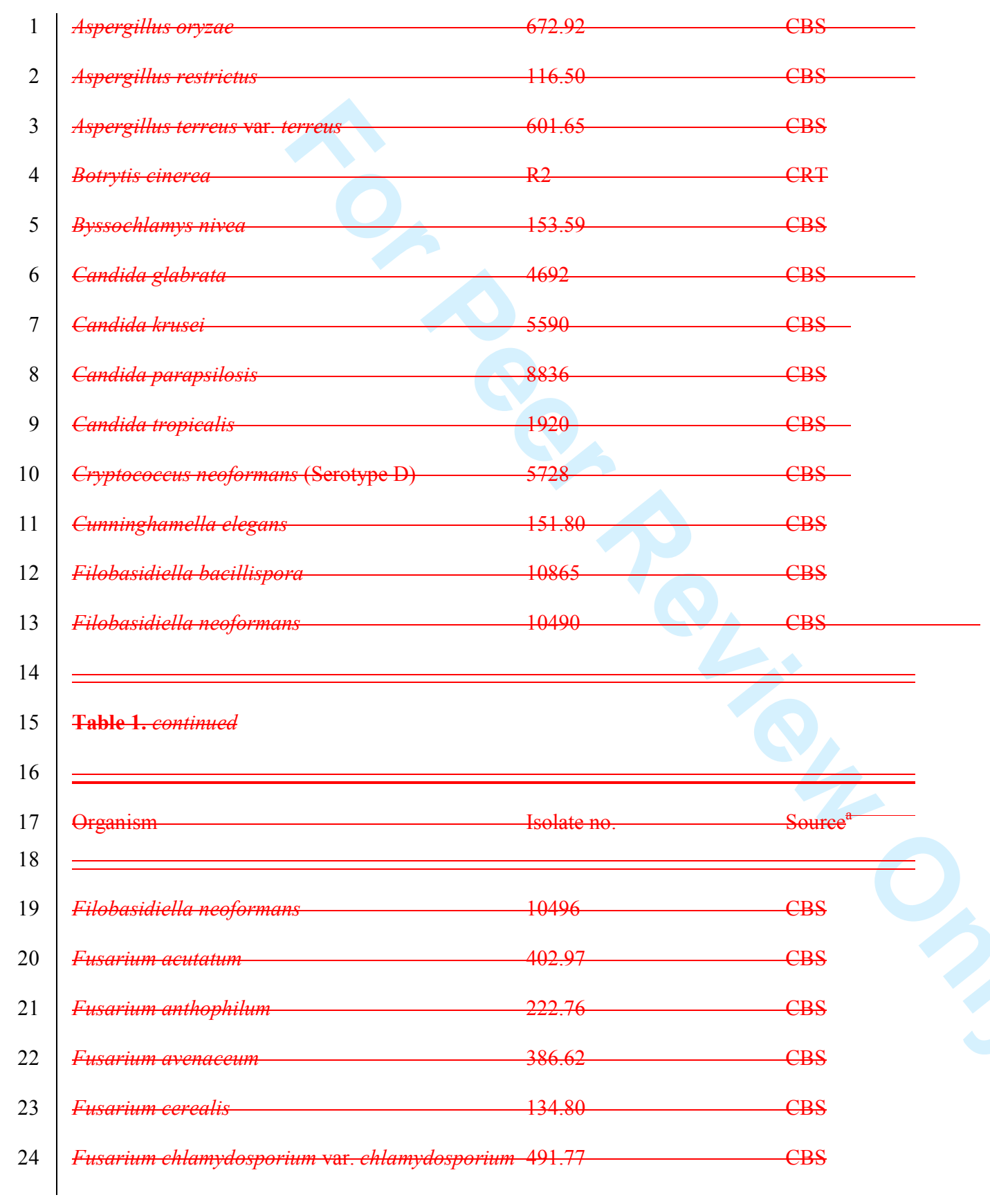

30 


\section{Page 31 of 87}

Fusarium-specific monoclonal antibody

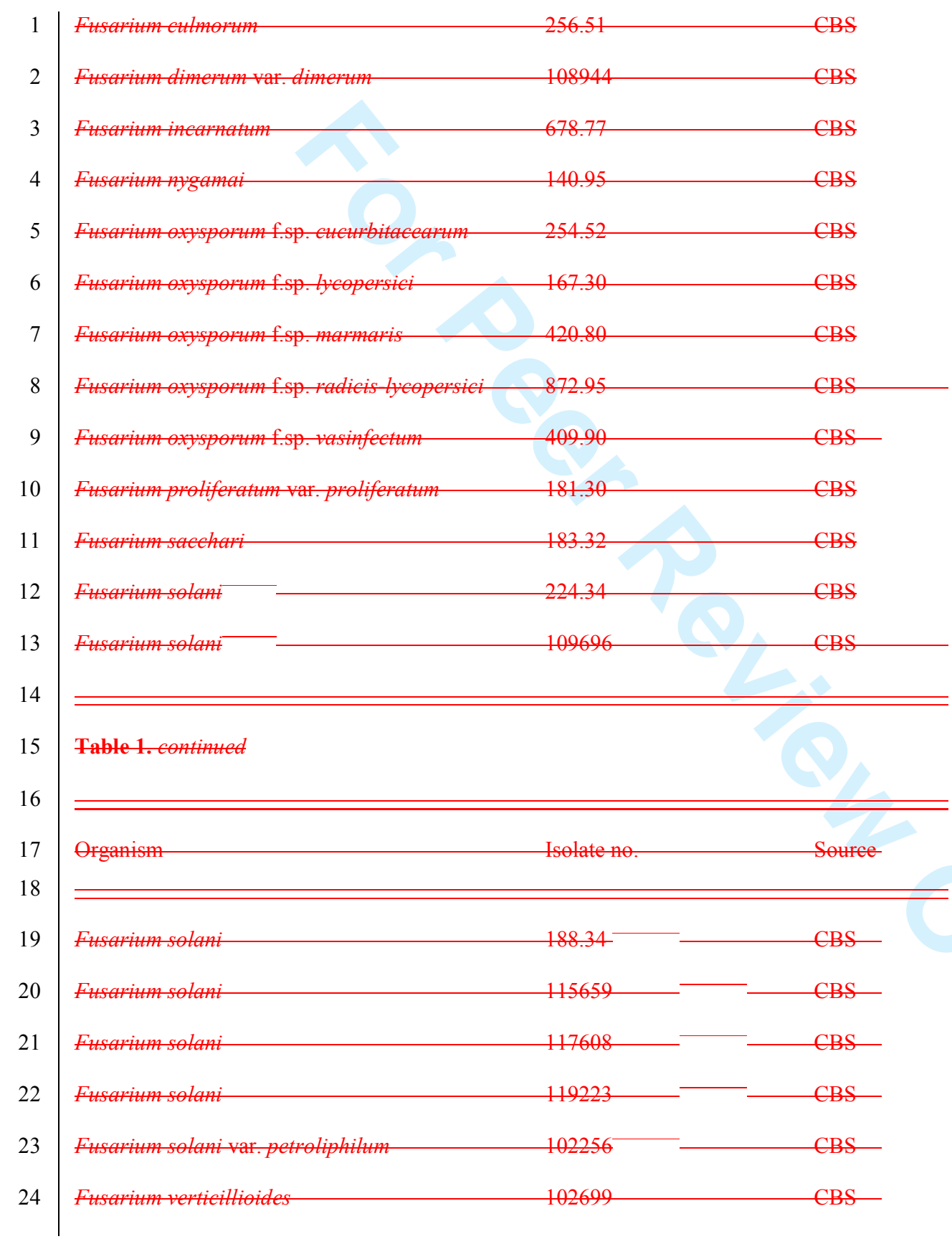


Fusarium-specific monoclonal antibody

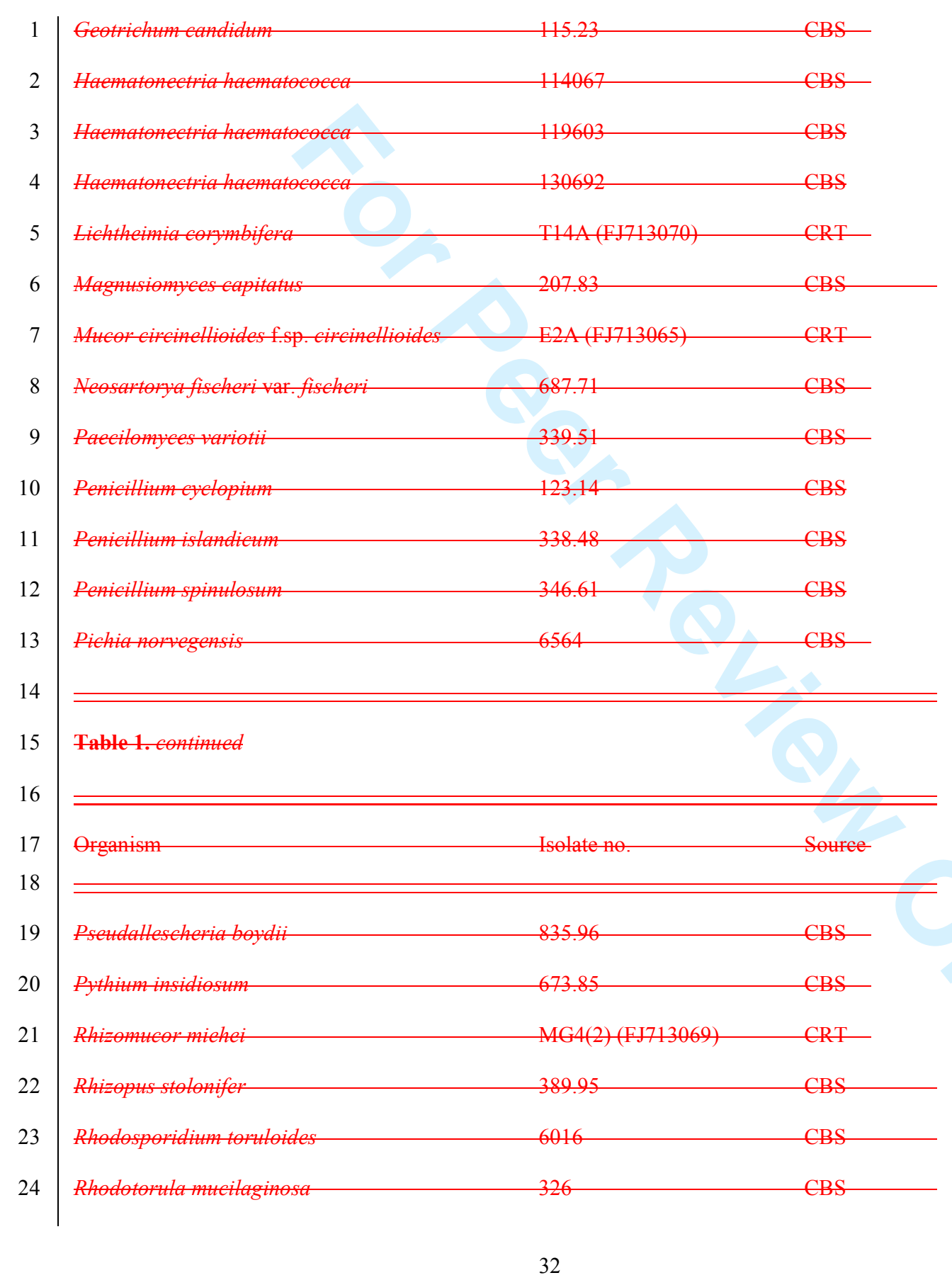




\section{Page 33 of 87}

Fusarium-specific monoclonal antibody

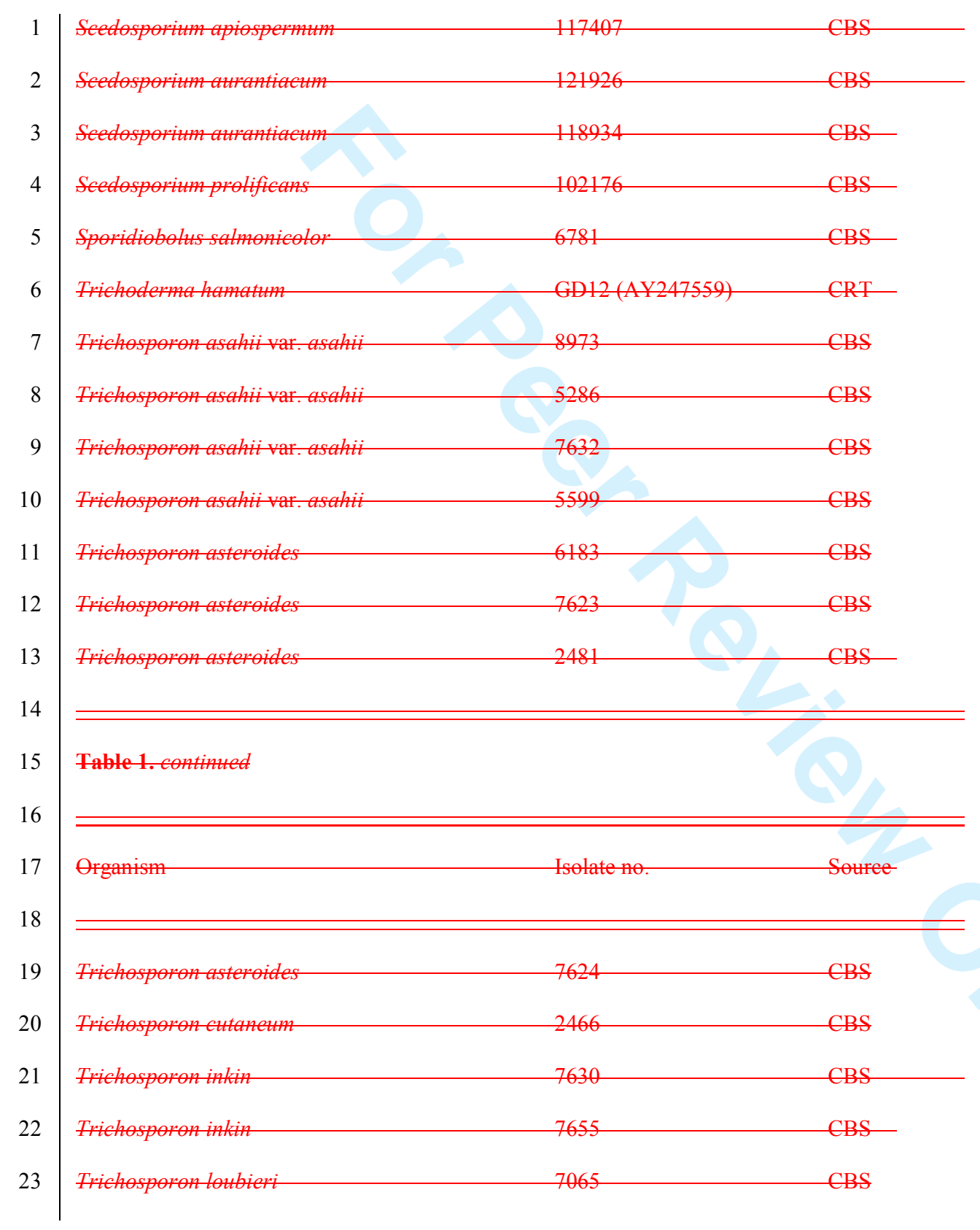


Fusarium-specific monoclonal antibody

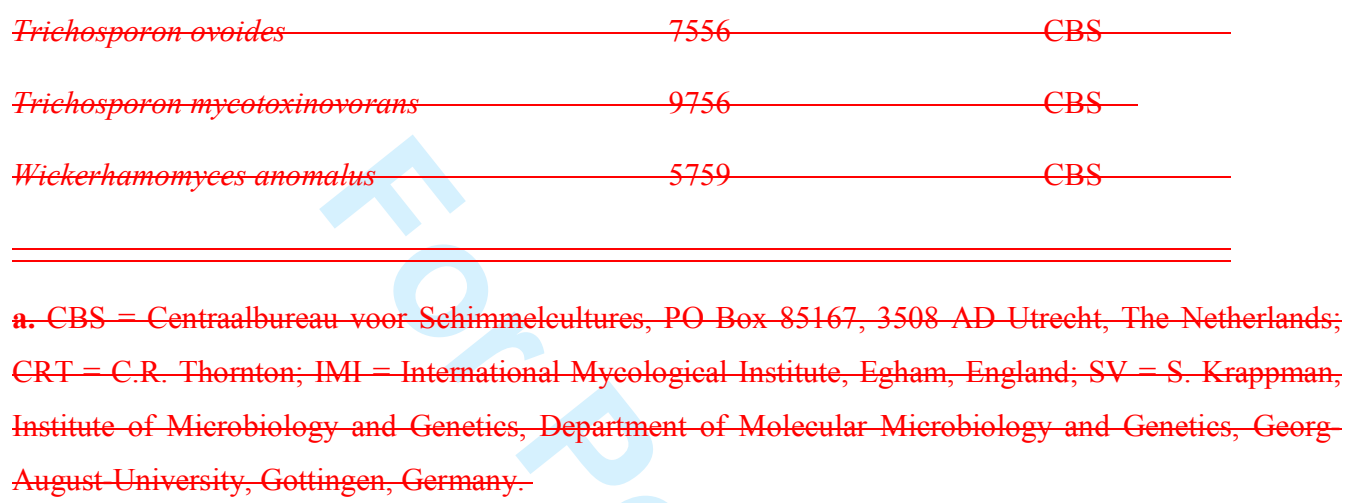

16 Table $\underline{12}$. Locations and identities of sink swabs and water samples and results of ELISA tests and fungal 17 identification based on ITS sequencing.

\begin{tabular}{|c|c|c|c|c|c|c|}
\hline $\begin{array}{l}\text { Location } \\
\text { (Source) }^{\text {a }}\end{array}$ & $\begin{array}{l}\text { Swab } \\
\text { ELISA abs } \\
(450 \mathrm{~nm})^{\mathrm{b}}\end{array}$ & $\begin{array}{c}\text { Mixed } \\
\text { culture } \\
\text { ELISA } \\
\text { abs } \\
(450 \mathrm{~nm})^{b}\end{array}$ & $\begin{array}{l}\text { Isolate } \\
\text { No. }\end{array}$ & $\begin{array}{c}\text { Axenic } \\
\text { culture } \\
\text { ELISA } \\
\text { abs } \\
(450 \mathrm{~nm})^{b}\end{array}$ & $\begin{array}{l}\text { Identification based on } \\
\text { ITS sequencing }\end{array}$ & $\begin{array}{c}\text { GenBank } \\
\text { Accession No. }\end{array}$ \\
\hline University (S1) & 0.0763 & 0.0575 & A1-1 & 1.3736 & Fusarium oxysporum & KT876668 \\
\hline University (S1) & & & A1-2 & 0.7646 & Fusarium oxysporum & KT876662 \\
\hline University (S1) & & & A1-3 & 0.0196 & Penicillium crustosum & KT876719 \\
\hline University (S1) & & & A1-4 & 0.0139 & Penicillium expansum & KT876718 \\
\hline University (S2) & 0.5472 & 0.0045 & A2-1 & 1.5723 & Fusarium solani & KT876635 \\
\hline University (S2) & & & A2-3 & 0.6554 & Fusarium oxysporum & KT876690 \\
\hline University (S2) & & & A2-5 & 1.1312 & Fusarium solani & KT876631 \\
\hline University (S3) & 0.0053 & 0.0077 & A3-1 & 0.0082 & Trichosporon domesticum & KT876717 \\
\hline University (S3) & & & A3-2 & 0.0162 & Cadophora fastigiata & KT876615 \\
\hline University (S3) & & & A3-3 & 0.0014 & Cyphellophora oxyspora & KT876613 \\
\hline
\end{tabular}




\begin{tabular}{|c|c|c|c|c|c|c|}
\hline University (S3) & & & A3-4 & 0.0093 & Penicillium crustosum & KT876714 \\
\hline University (S4) & 0.0297 & 0.0049 & A4-1 & 0.0105 & Phoma herbarum & KT876697 \\
\hline University (S4) & & & A4-2 & 0.0079 & Penicillium echinulatum & KT876710 \\
\hline University (S4) & & & A4-3 & 0.0029 & Cytobasidium slooffiae & KT876704 \\
\hline University (S4) & & & A4-6 & 0.0060 & Trichoderma asperellum & KT876620 \\
\hline University (S5) & 0.0206 & 0.0039 & A5-1 & 1.1815 & Fusarium oxysporum & KT876692 \\
\hline University (S5) & & & A5-2 & 0.0034 & Penicillium crustosum & KT876715 \\
\hline University (S5) & & & A5-5 & 0.0088 & Aspergillus niger & KT876702 \\
\hline University (S5) & & & A5-6 & 1.0630 & Fusarium oxysporum & KT876667 \\
\hline University (S5) & & & A5-7 & 0.0037 & $\begin{array}{l}\text { Rhodotorula- } \\
\text { mucilaginosa }\end{array}$ & KT876700 \\
\hline University (S5) & & & A5-8 & 0.0088 & Cyphellophora oxyspora & KT876614 \\
\hline University (S6) & 0.0251 & 0.0412 & A6-1 & 1.1130 & Fusarium oxysporum & KT876648 \\
\hline University (S6) & & & A6-2 & 0.9410 & Fusarium oxysporum & KT876678 \\
\hline University (S6) & & & A6-3 & 0.6377 & Fusarium oxysporum & KT876688 \\
\hline University (S6) & & & A6-4 & 1.0020 & Fusarium solani & KT876640 \\
\hline University (S7) & 0.1716 & 0.0082 & A7-1 & 0.0098 & Exophiala pisciphila & KT876618 \\
\hline University (S7) & & & A7-2 & 0.0096 & $\begin{array}{c}\text { Penicillium } \\
\text { brevicompactum }\end{array}$ & KT876695 \\
\hline University (S7) & & & A7-3 & 1.0556 & Fusarium oxysporum & KT876684 \\
\hline University (S7) & & & A7-4 & 1.0077 & Fusarium oxysporum & KT876671 \\
\hline University (S8) & 0.0431 & 0.0008 & A8-1 & 1.1045 & Fusarium oxysporum & KT876672 \\
\hline University (S8) & & & A8-2 & 0.9707 & Fusarium oxysporum & KT876654 \\
\hline University (S8) & & & A8-3 & 0.0091 & Trichoderma atroviride & KT876622 \\
\hline University (S9) & 0.3806 & 0.0052 & A9-1 & 0.9087 & Fusarium solani & KT876639 \\
\hline University (S9) & & & A9-2 & 0.8849 & Fusarium solani & KT876638 \\
\hline University (S9) & & & A9-3 & 0.9004 & Fusarium solani & KT876632 \\
\hline University (S9) & & & A9-4 & 0.8093 & Fusarium solani & KT876636 \\
\hline University (S10) & 0.0034 & 0.0028 & A10-1 & 0.0188 & Rhodotorula slooffiae & \\
\hline University (S10) & & & A10-2 & 0.0020 & Exophiala pisciphila & KT876616 \\
\hline University (S11) & 0.0231 & 0.0005 & B1-1 & 0.6064 & Fusarium dimerum & KT876625 \\
\hline University (S11) & & & B1-6 & 0.8138 & Fusarium dimerum & KT876628 \\
\hline University (S12) & 0.0200 & 0.0020 & B2-1 & 0.5678 & Fusarium dimerum & KT876626 \\
\hline University (S12) & & & B2-5 & 0.4827 & Fusarium dimerum & KT876624 \\
\hline University (S13) & 0.0091 & 0.0023 & B3-4 & 0.0026 & Phoma herbarum & KT876696 \\
\hline University (S14) & 0.0163 & 0.0008 & B4-1 & 0.6992 & Fusarium dimerum & KT876627 \\
\hline University (S15) & 0.0132 & 0.0003 & B5-1 & 0.8008 & Fusarium oxysporum & KT876674 \\
\hline University (S15) & & & B5-2 & 0.0048 & Mucor circinelloides & KT876701 \\
\hline University (S15) & & & B5-3 & 0.8851 & Fusarium oxysporum & KT876677 \\
\hline University (S16) & 0.0229 & 0.0002 & B6-1 & 0.8193 & Fusarium oxysporum & KT876676 \\
\hline University (S16) & & & B6-2 & 0.7582 & Fusarium oxysporum & KT876661 \\
\hline University (S17) & 0.0395 & 0.0014 & B7-1 & 0.8201 & Fusarium oxysporum & KT876675 \\
\hline University (S17) & & & B7-6 & 0.7758 & Fusarium dimerum & KT876623 \\
\hline University (S18) & 0.0133 & 0.0011 & B8-1 & 0.7347 & Fusarium solani & KT876637 \\
\hline University (S18) & & & B8-7 & 0.0095 & Rhodosporidium babjevae & KT876706 \\
\hline University (S19) & 0.0212 & 0.0048 & B9-1 & 0.0051 & $\begin{array}{c}\text { Meyerozyma } \\
\text { guilliermondii }\end{array}$ & KT876707 \\
\hline University (S19) & & & B9-2 & 0.8104 & Fusarium oxysporum & KT876657 \\
\hline
\end{tabular}

Formatted: None, Indent: Left: 0", First line: $0 "$, Don't keep with next 
Fusarium-specific monoclonal antibody

\begin{tabular}{|c|c|c|c|c|c|c|}
\hline University (S19) & & & B9-3 & 0.6302 & Fusarium solani & KT876634 \\
\hline University (S19) & & & B9-5 & 0.0017 & Penicillium crustosum & KT876720 \\
\hline University (S20) & 0.5467 & 0.0394 & B10-1 & 0.7467 & Fusarium oxysporum & KT876680 \\
\hline University (S20) & & & B10-2 & 0.7667 & Fusarium oxysporum & KT876659 \\
\hline University (S20) & & & B10-6 & 0.0071 & Candida parapsilosis & KT876703 \\
\hline University (S20) & & & B10-7 & 0.0251 & $\begin{array}{l}\text { Meyerozyma } \\
\text { guilliermondii }\end{array}$ & KT876711 \\
\hline University (S20) & & & B10-9 & 0.0031 & Cystobasidium slooffiae & KT876712 \\
\hline University (S20) & & & B10-12 & 0.0251 & Trichosporon asteroides & KT876713 \\
\hline University (S21) & 0.0083 & 0.0269 & $\mathrm{C} 1-1$ & 0.0044 & Clavispora lusitaniae & KT876708 \\
\hline University (S21) & & 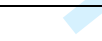 & $\mathrm{C} 1-3$ & 0.8875 & Fusarium oxysporum & KT876682 \\
\hline University (S21) & & & $\mathrm{C} 1-4$ & 0.8884 & Fusarium dimerum & KT876629 \\
\hline University (S21) & & & $\mathrm{C} 1-7$ & 0.0003 & Exophiala pisciphila & KT876617 \\
\hline University (S22) & 0.2977 & 0.0047 & $\mathrm{C} 2-1$ & 0.9443 & Fusarium oxysporum & KT876658 \\
\hline University (S22) & & & $\mathrm{C} 2-4$ & 0.9825 & Fusarium oxysporum & KT876694 \\
\hline University (S22) & & & $\mathrm{C} 2-7$ & 0.8677 & Fusarium oxysporum & KT876687 \\
\hline University (S23) & 0.0808 & 0.0116 & C3-1 & 0.9853 & Fusarium oxysporum & KT876683 \\
\hline University (S23) & & & $\mathrm{C} 3-2$ & 1.0160 & Fusarium oxysporum & KT876693 \\
\hline University (S23) & & & $\mathrm{C} 3-4$ & 0.7485 & Fusarium oxysporum & KT876644 \\
\hline University (S24) & 0.1113 & 0.0202 & C4-1 & 0.8930 & Fusarium oxysporum & KT876670 \\
\hline University (S24) & & & $\mathrm{C} 4-2$ & 0.0028 & Candida intermedia & KT876709 \\
\hline University (S24) & & & C4-5 & 0.9008 & Fusarium dimerum & KT876630 \\
\hline University (S25) & 0.5741 & 0.0097 & C5-1 & 0.9874 & Fusarium oxysporum & KT876652 \\
\hline University (S25) & & & C5-2 & 1.0996 & Fusarium oxysporum & KT876656 \\
\hline University (S25) & & & C5-3 & 1.1236 & Fusarium oxysporum & KT876649 \\
\hline University (S25) & & & C5-4 & 1.0355 & Fusarium oxysporum & KT876669 \\
\hline University (S25) & & & $\mathrm{C} 5-5$ & 1.0672 & Fusarium oxysporum & KT876660 \\
\hline University (S26) & 0.0603 & 0.0348 & C6-1 & 1.0135 & Fusarium oxysporum & KT876665 \\
\hline University (S26) & & & C6-2 & 0.9362 & Fusarium oxysporum & KT876681 \\
\hline University (S26) & & & C6-3 & 0.8898 & Fusarium oxysporum & \\
\hline University (S26) & & & C6-4 & 1.0577 & Fusarium oxysporum & KT876686 \\
\hline University (S26) & & & C6-5 & 0.9445 & Fusarium oxysporum & KT876646 \\
\hline University (S27) & 0.1279 & 0.0113 & C7-1 & 0.9724 & Fusarium oxysporum & KT876666 \\
\hline University (S28) & 0.0607 & 0.0035 & C8-1 & 0.8694 & Fusarium oxysporum & KT876685 \\
\hline University (S28) & & & $\mathrm{C} 8-2$ & 0.9599 & Fusarium oxysporum & KT876655 \\
\hline University (S28) & & & C8-3 & 0.0031 & Penicillium expansum & KT876716 \\
\hline University (S28) & & & $\mathrm{C} 8-4$ & 0.0049 & Phoma herbarum & KT876698 \\
\hline University (S28) & & & C8-6 & 0.9003 & Fusarium oxysporum & KT876679 \\
\hline University (S29) & 0.0310 & 0.0001 & C9-1 & 1.0488 & Fusarium oxysporum & KT876651 \\
\hline University (S29) & & & C9-2 & 0.9254 & Fusarium oxysporum & KT876645 \\
\hline University (S29) & & & C9-3 & 0.9711 & Fusarium oxysporum & KT876664 \\
\hline University (S29) & & & C9-4 & 1.035 & Fusarium oxysporum & KT876647 \\
\hline University (S29) & & & C9-5 & 0.9604 & Fusarium oxysporum & KT876663 \\
\hline University (S30) & 0.1002 & 0.0333 & C10-1 & 0.9254 & Fusarium oxysporum & KT876689 \\
\hline University (S30) & & & C10-2 & 1.0198 & Fusarium solani & KT876642 \\
\hline University (S30) & & & C10-4 & 0.9007 & Fusarium solani & KT876641 \\
\hline University (S30) & & & $\mathrm{C} 10-7$ & 1.0697 & Fusarium solani & KT876643 \\
\hline University (S31) & 0.6175 & 0.1129 & CRT1-1 & 0.4639 & Fusarium oxysporum & KT876691 \\
\hline University (S31) & & & CRT1-2 & 0.9086 & Fusarium oxysporum & KT876650 \\
\hline
\end{tabular}


Fusarium-specific monoclonal antibody

\begin{tabular}{|c|c|c|c|c|c|c|}
\hline University (S31) & & & CRT1-3 & 0.7503 & Fusarium oxysporum & KT876652 \\
\hline University (S32) & 0.0303 & 0.0021 & CRT2-1 & 0.0040 & Trichoderma asperellum & KT876619 \\
\hline University (S32) & & & CRT2-2 & 0.0417 & Trichoderma asperellum & KT876621 \\
\hline University (S33) & 0.0663 & 0.0028 & CRT3-1 & 0.0256 & Phoma herbarum & KT876699 \\
\hline ICU (S34) & 0.0285 & 0.3620 & R1-1 & 1.5057 & Fusarium solani & KT876550 \\
\hline ICU (S34) & & & R1-2 & 1.5154 & Fusarium solani & KT876551 \\
\hline ICU (S34) & & & R1-3 & 1.5555 & Fusarium solani & KT876549 \\
\hline ICU (S35) & 0.0071 & 0.0147 & $\mathrm{R} 2-4$ & 0.0096 & Clonostachys rosea & KT876552 \\
\hline ICU (S35) & & & R2-5 & 0.0018 & Clonostachys rosea & KT876553 \\
\hline ICU (S35) & & & R2-6 & 0.0035 & Clonostachys rosea & KT876554 \\
\hline ICU (S36) & 0.0391 & 0.045 & R3-1 & 0.0006 & Trichoderma asperellum & KT876548 \\
\hline ITU (S37) & 0.7291 & 0.7724 & R5-1 & 1.5606 & Fusarium dimerum & KT876561 \\
\hline ITU (S37) & & & R5-2 & 1.3481 & Fusarium dimerum & KT876565 \\
\hline ITU (S37) & & & R5-3 & 1.4990 & Fusarium dimerum & KT876567 \\
\hline ITU (S38) & 1.2086 & 0.3691 & R6-1 & 1.3388 & Fusarium dimerum & KT876572 \\
\hline ITU (S38) & & & R6-2 & 1.4734 & Fusarium dimerum & KT876563 \\
\hline ITU (S38) & & & R6-3 & 1.4312 & Fusarium dimerum & KT876562 \\
\hline ITU (S38) & & & R6-9 & 1.2648 & Fusarium oxysporum & KT876557 \\
\hline ITU (S39) & 0.1121 & 0.8186 & R7-1 & 1.3266 & Fusarium dimerum & KT876570 \\
\hline ITU (S39) & & & R7-2 & 1.2352 & Fusarium dimerum & KT876564 \\
\hline ITU (S40) & 1.0157 & 0.3612 & R8-1 & 1.2399 & Fusarium dimerum & KT876568 \\
\hline ITU (S40) & & & R8-2 & 1.1552 & Fusarium dimerum & KT876566 \\
\hline ITU (S40) & & & R8-3 & 1.1856 & Fusarium dimerum & KT876558 \\
\hline ITU (S41) & 0.0391 & 0.0477 & R9-3 & 0.0113 & $\begin{array}{c}\text { Exophiala } \\
\text { phaeomuriformis }\end{array}$ & KT876555 \\
\hline ITU (S42) & 0.0549 & 0.0097 & R10-2 & 0.0072 & Cadophora fastigiata & KT876556 \\
\hline ITU (S42) & & & R10-6 & 0.0124 & Coniochaeta fasciculata & KT876721 \\
\hline ITU (S43) & 0.0630 & 1.5751 & $\mathrm{X} 2-2$ & 1.1492 & Fusarium dimerum & KT876571 \\
\hline ITU (S43) & & & $\mathrm{X} 2-4$ & 1.2505 & Fusarium dimerum & KT876560 \\
\hline ITU (S43) & & & $\mathrm{X} 2-5$ & 1.1397 & Fusarium dimerum & KT876569 \\
\hline ITU (S43) & & & $\mathrm{X} 2-6$ & 1.1716 & Fusarium dimerum & KT876559 \\
\hline $\begin{array}{l}\text { Ophthalmology } \\
\text { Unit (S44) }\end{array}$ & 1.0313 & 1.5035 & X3-1 & 1.1353 & Fusarium dimerum & KT876509 \\
\hline $\begin{array}{l}\text { Ophthalmology } \\
\text { Unit (S44) }\end{array}$ & & & $\mathrm{X} 3-2$ & 0.0224 & Candida parapsilosis & KT876498 \\
\hline $\begin{array}{l}\text { Ophthalmology } \\
\text { Unit (S44) }\end{array}$ & & & $\mathrm{X} 3-3$ & 1.1856 & Fusarium dimerum & KT876512 \\
\hline $\begin{array}{l}\text { Ophthalmology } \\
\text { Unit (S45) }\end{array}$ & 1.4582 & 1.4049 & $\mathrm{X} 4-1$ & 1.1322 & Fusarium dimerum & KT876511 \\
\hline $\begin{array}{l}\text { Ophthalmology } \\
\text { Unit (S45) }\end{array}$ & & & $\mathrm{X} 4-2$ & 1.1523 & Fusarium dimerum & KT876500 \\
\hline $\begin{array}{l}\text { Ophthalmology } \\
\text { Unit (S45) }\end{array}$ & & & $\mathrm{X} 4-3$ & 0.0622 & Candida parapsilosis & KT876508 \\
\hline $\begin{array}{l}\text { Ophthalmology } \\
\text { Unit (S45) }\end{array}$ & & & $\mathrm{X} 4-4$ & 0.1245 & Gloeotinia temulenta & KT876515 \\
\hline $\begin{array}{l}\text { Ophthalmology } \\
\text { Unit (S46) }\end{array}$ & 0.3325 & 1.4466 & $\mathrm{X} 5-2$ & 1.0654 & Fusarium dimerum & KT876510 \\
\hline $\begin{array}{l}\text { Ophthalmology } \\
\text { Unit (S46) }\end{array}$ & & & $\mathrm{X} 5-3$ & 0.0599 & $\begin{array}{l}\text { Rhodotorula } \\
\text { mucilaginosa }\end{array}$ & KT876501 \\
\hline
\end{tabular}


Fusarium-specific monoclonal antibody

\begin{tabular}{|c|c|c|c|c|c|c|}
\hline $\begin{array}{l}\text { Ophthalmology } \\
\text { Unit (S46) }\end{array}$ & & & $\mathrm{X} 5-4$ & 1.0862 & Fusarium dimerum & KT876513 \\
\hline $\begin{array}{l}\text { Ophthalmology } \\
\text { Unit (S47) }\end{array}$ & 0.2640 & 1.3729 & X6-1 & 0.0616 & Candida parapsilosis & KT876499 \\
\hline $\begin{array}{l}\text { Ophthalmology } \\
\text { Unit (S47) }\end{array}$ & & & X6-2 & 0.063 & Engyodontium album & KT876522 \\
\hline $\begin{array}{l}\text { Ophthalmology } \\
\text { Unit (S47) }\end{array}$ & & & $\mathrm{X} 6-3$ & 0.0603 & Engyodontium album & KT876521 \\
\hline $\begin{array}{l}\text { Ophthalmology } \\
\text { Unit (S47) }\end{array}$ & & & X6-4 & 0.0657 & $\begin{array}{c}\text { Exophiala } \\
\text { phaeomuriformis }\end{array}$ & KT876504 \\
\hline $\begin{array}{l}\text { Ophthalmology } \\
\text { Unit (S48) }\end{array}$ & 0.5299 & 0.0223 & $\mathrm{X} 8-2$ & 0.0118 & $\begin{array}{l}\text { Cladosporium } \\
\text { macrocarpum }\end{array}$ & KT876506 \\
\hline $\begin{array}{l}\text { Ophthalmology } \\
\text { Unit (S48) }\end{array}$ & & & $X 8-3$ & 0.0985 & Gloeotinia temulenta & KT876514 \\
\hline $\begin{array}{l}\text { Ophthalmology } \\
\text { Unit (S48) }\end{array}$ & & & $X 8-4$ & 0.0662 & Engyodontium album & KT876523 \\
\hline $\begin{array}{l}\text { Ophthalmology } \\
\text { Unit (S49) }\end{array}$ & 0.1872 & 0.0061 & X9-1 & 0.0686 & Engyodontium album & KT876520 \\
\hline $\begin{array}{l}\text { Ophthalmology } \\
\text { Unit (S49) }\end{array}$ & & & X9-2 & 0.0611 & Candida parapsilosis & KT876496 \\
\hline $\begin{array}{l}\text { Ophthalmology } \\
\text { Unit (S49) }\end{array}$ & & & X9-3 & 0.0641 & Candida parapsilosis & KT876497 \\
\hline $\begin{array}{l}\text { Ophthalmology } \\
\text { Unit (S49) }\end{array}$ & & & X9-4 & 0.0677 & Exophiala dermatitidis & KT876503 \\
\hline $\begin{array}{l}\text { Ophthalmology } \\
\text { Unit (S49) }\end{array}$ & & & X9-5 & 0.0705 & Engyodontium album & KT876519 \\
\hline $\begin{array}{l}\text { Ophthalmology } \\
\text { Unit (S49) }\end{array}$ & & & X9-7 & 0.0660 & Exophiala pisciphila & KT876502 \\
\hline $\begin{array}{l}\text { Ophthalmology } \\
\text { Unit (T1) }\end{array}$ & 0.0132 & 0.0159 & X10-1 & 0.0868 & $\begin{array}{c}\text { Cladosporium } \\
\text { sphaerospermum }\end{array}$ & KT876507 \\
\hline $\begin{array}{l}\text { Ophthalmology } \\
\text { Unit (T1) }\end{array}$ & & & $\mathrm{X} 10-3$ & 0.0130 & Engyodontium album & KT876518 \\
\hline $\begin{array}{l}\text { Ophthalmology } \\
\text { Unit (T2) }\end{array}$ & 0.0117 & 0.0883 & W1-1 & 0.0114 & Paraconiothyrium fuckelii & KT876505 \\
\hline $\begin{array}{l}\text { Ophthalmology } \\
\text { Unit (T2) }\end{array}$ & & & W1-2 & 0.0139 & Engyodontium album & KT876517 \\
\hline $\begin{array}{l}\text { Ophthalmology } \\
\text { Unit (T2) }\end{array}$ & & & W1-3 & 0.0108 & Engyodontium album & KT876516 \\
\hline $\begin{array}{l}\text { Haematology } \\
\text { Unit (S50) }\end{array}$ & 0.2694 & 0.0093 & $\mathrm{~K} 1-1$ & 0.0172 & Trichoderma asperellum & KT876534 \\
\hline $\begin{array}{l}\text { Haematology } \\
\text { Unit (S51) }\end{array}$ & 0.7466 & 1.1746 & K2-1 & 0.0144 & Trichoderma viride & KT876533 \\
\hline $\begin{array}{l}\text { Haematology } \\
\text { Unit (S51) }\end{array}$ & & & $\mathrm{K} 2-3$ & 1.1385 & Fusarium solani & KT876543 \\
\hline $\begin{array}{l}\text { Haematology } \\
\text { Unit (S51) }\end{array}$ & & & $\mathrm{K} 2-4$ & 1.2487 & Fusarium solani & KT876542 \\
\hline $\begin{array}{l}\text { Haematology } \\
\text { Unit (S52) }\end{array}$ & 1.0621 & 1.3090 & K6-1 & 1.1544 & Fusarium oxysporum & KT876541 \\
\hline Haematology & & & K6-4 & 0.0071 & Candida parapsilosis & KT876524 \\
\hline
\end{tabular}


Fusarium-specific monoclonal antibody

\begin{tabular}{|c|c|c|c|c|c|c|}
\hline Unit (S52) & & & & & & \\
\hline $\begin{array}{c}\text { Haematology } \\
\text { Unit (S53) }\end{array}$ & 1.0120 & 1.2628 & K8-1 & 1.2376 & Fusarium solani & KT876722 \\
\hline $\begin{array}{l}\text { Haematology } \\
\text { Unit (S53) }\end{array}$ & & & $\mathrm{K} 8-2$ & 1.2983 & Fusarium solani & KT876723 \\
\hline $\begin{array}{c}\text { Haematology } \\
\text { Unit (S53) }\end{array}$ & & & K8-6 & 0.0084 & Cadophora fastigiata & KT876531 \\
\hline $\begin{array}{c}\text { Haematology } \\
\text { Unit (S53) }\end{array}$ & & & K8-7 & 0.0051 & Trichoderma asperellum & KT876535 \\
\hline $\begin{array}{c}\text { Haematology } \\
\text { Unit (S54) }\end{array}$ & 0.9843 & 1.2903 & K9-1 & 0.0046 & Candida parapsilosis & KT876525 \\
\hline $\begin{array}{c}\text { Haematology } \\
\text { Unit (S54) }\end{array}$ & & & K9-2 & 0.0090 & Rhodotorula dairenensis & KT876526 \\
\hline $\begin{array}{c}\text { Haematology } \\
\text { Unit (S54) }\end{array}$ & & & K9-4 & 0.0073 & Cadophora fastigiata & KT876532 \\
\hline $\begin{array}{c}\text { Haematology } \\
\text { Unit (S54) }\end{array}$ & & & K9-5 & 1.1652 & Fusarium solani & KT876547 \\
\hline $\begin{array}{c}\text { Haematology } \\
\text { Unit (S54) }\end{array}$ & & & K9-6 & 1.0316 & Fusarium solani & KT876545 \\
\hline $\begin{array}{c}\text { Haematology } \\
\text { Unit (S55) }\end{array}$ & 0.8638 & 1.0753 & K10-1 & 1.0684 & Fusarium solani & KT876546 \\
\hline $\begin{array}{c}\text { Haematology } \\
\text { Unit (S55) }\end{array}$ & & & $\mathrm{K} 10-2$ & 1.0386 & Fusarium solani & KT876544 \\
\hline $\begin{array}{c}\text { Haematology } \\
\text { Unit (W50) }\end{array}$ & 0.0082 & 0.0451 & G1-2 & 0.0097 & Exophiala pisciphila & KT876529 \\
\hline $\begin{array}{c}\text { Haematology } \\
\text { Unit (W51) }\end{array}$ & 0.0581 & 0.0553 & G2-1 & 0.0104 & Engyodontium album & KT876540 \\
\hline $\begin{array}{c}\text { Haematology } \\
\text { Unit (W51) }\end{array}$ & & & $\mathrm{G} 2-3$ & 0.0062 & Exophiala pisciphila & KT876530 \\
\hline $\begin{array}{c}\text { Haematology } \\
\text { Unit (W52) }\end{array}$ & 0.0125 & 0.0118 & G6-2 & 0.0161 & Exophiala castellanii & КT876528 \\
\hline $\begin{array}{l}\text { Haematology } \\
\text { Unit (W53) }\end{array}$ & 0.0075 & 0.0219 & G8-1 & 0.0065 & Engyodontium album & KT876538 \\
\hline $\begin{array}{l}\text { Haematology } \\
\text { Unit (W54) }\end{array}$ & 0.0002 & 0.0164 & G9-2 & 0.0005 & Gloeotinia temulenta & KT876537 \\
\hline $\begin{array}{l}\text { Haematology } \\
\text { Unit (W55) }\end{array}$ & 0.0021 & 0.0182 & G10-1 & 0.0051 & Engyodontium album & KT876539 \\
\hline $\begin{array}{l}\text { Oncology Unit } \\
\text { (S56) }\end{array}$ & 0.4822 & 1.2287 & H1-1 & 0.9022 & Fusarium dimerum & KT876595 \\
\hline $\begin{array}{l}\text { Oncology Unit } \\
\text { (S56) }\end{array}$ & & & H1-3 & 0.0151 & Pichia kudriavzevii & KT876578 \\
\hline $\begin{array}{l}\text { Oncology Unit } \\
\text { (S57) }\end{array}$ & 1.0579 & 1.1650 & $\mathrm{H} 2-1$ & 0.9312 & Fusarium dimerum & KT876590 \\
\hline $\begin{array}{l}\text { Oncology Unit } \\
\text { (S57) }\end{array}$ & & & $\mathrm{H} 2-5$ & 0.0061 & Magnusiomyces capitatus & KT876611 \\
\hline $\begin{array}{l}\text { Oncology Unit } \\
\text { (S58) }\end{array}$ & 1.0938 & 1.2091 & H3-1 & 0.8909 & Fusarium oxysporum & KT876584 \\
\hline $\begin{array}{l}\text { Oncology Unit } \\
\text { (S58) }\end{array}$ & & & H3-4 & 0.0075 & Candida palmioleophila & KT876573 \\
\hline Oncology Unit & & & H3-5 & 0.0140 & Rhodotorula glutinis & KT876598 \\
\hline
\end{tabular}


Fusarium-specific monoclonal antibody

\begin{tabular}{|c|c|c|c|c|c|c|}
\hline & & & & & & \\
\hline (S58) & & & & & & \\
\hline $\begin{array}{c}\text { Oncology Unit } \\
\text { (S59) }\end{array}$ & 0.0253 & 1.0856 & H4-1 & 0.8884 & Fusarium dimerum & КT876596 \\
\hline $\begin{array}{c}\text { Oncology Unit } \\
\text { (S59) }\end{array}$ & & & H4-3 & 0.0004 & Magnusiomyces capitatus & KT876612 \\
\hline $\begin{array}{l}\text { Oncology Unit } \\
\text { (S60) }\end{array}$ & 1.0856 & 1.0193 & H5-1 & 1.1148 & Fusarium dimerum & KT876593 \\
\hline $\begin{array}{c}\text { Oncology Unit } \\
\text { (S60) }\end{array}$ & & & H5-3 & 0.005 & Candida tropicalis & КT876574 \\
\hline $\begin{array}{c}\text { Oncology Unit } \\
\text { (S61) }\end{array}$ & 0.5256 & 1.1898 & H6-1 & 1.1238 & Fusarium dimerum & КT876594 \\
\hline $\begin{array}{c}\text { Oncology Unit } \\
\text { (S62) }\end{array}$ & 1.0789 & 1.1588 & $\mathrm{H} 7-1$ & 1.1513 & Fusarium oxysporum & KT876583 \\
\hline $\begin{array}{c}\text { Oncology Unit } \\
\text { (S62) }\end{array}$ & & & $\mathrm{H} 7-3$ & 0.0045 & Candida tropicalis & KT876575 \\
\hline $\begin{array}{c}\text { Oncology Unit } \\
\text { (S63) }\end{array}$ & 0.0953 & 0.0152 & H8-1 & 0.0063 & Phoma herbarum & KT876580 \\
\hline $\begin{array}{c}\text { Oncology Unit } \\
\text { (S63) }\end{array}$ & & & H8-3 & 0.0126 & Candida albicans & KT876577 \\
\hline $\begin{array}{c}\text { Oncology Unit } \\
\text { (S63) }\end{array}$ & & & $\mathrm{H} 8-4$ & 0.0088 & $\begin{array}{l}\text { Rhodotorula } \\
\text { mucilaginosa }\end{array}$ & KT876599 \\
\hline $\begin{array}{c}\text { Oncology Unit } \\
\text { (S64) }\end{array}$ & 0.1926 & 0.0162 & H9-1 & 0.0152 & Exophiala dermatitidis & KT876581 \\
\hline $\begin{array}{c}\text { Oncology Unit } \\
\text { (S64) }\end{array}$ & & & H9-2 & 0.0049 & Candida orthopsilosis & KT876576 \\
\hline $\begin{array}{c}\text { Oncology Unit } \\
\text { (S64) }\end{array}$ & & & H9-3 & 0.0109 & Pichia kudriavzevii & KT876579 \\
\hline $\begin{array}{c}\text { Oncology Unit } \\
\text { (S65) }\end{array}$ & 0.4961 & 1.1005 & H10-1 & 0.9606 & Fusarium dimerum & KT876589 \\
\hline $\begin{array}{l}\text { Oncology Unit } \\
\text { (S65) }\end{array}$ & & & H10-4 & 0.0163 & Trametes ochracea & KT876608 \\
\hline $\begin{array}{l}\text { Oncology Unit } \\
\text { (W56) }\end{array}$ & 0.0030 & 0.0247 & P1-2 & 0.0082 & Exophiala dermatitidis & KT876582 \\
\hline $\begin{array}{l}\text { Oncology Unit } \\
\text { (W56) }\end{array}$ & & & P1-3 & 0.0024 & Trametes versicolor & KT876603 \\
\hline $\begin{array}{l}\text { Oncology Unit } \\
\text { (W57) }\end{array}$ & 0.0061 & 1.0891 & P2-1 & 0.8451 & Fusarium dimerum & KT876587 \\
\hline $\begin{array}{l}\text { Oncology Unit } \\
\text { (W57) }\end{array}$ & & & $\mathrm{P} 2-2$ & 0.9429 & Fusarium dimerum & KT876597 \\
\hline $\begin{array}{l}\text { Oncology Unit } \\
\text { (W57) }\end{array}$ & & & P2-3 & 0.8627 & Fusarium dimerum & KT876588 \\
\hline $\begin{array}{l}\text { Oncology Unit } \\
\text { (W58) }\end{array}$ & 0.0016 & 0.0202 & P3-1 & 0.0177 & Trametes ochracea & KT876607 \\
\hline $\begin{array}{l}\text { Oncology Unit } \\
\text { (W59) }\end{array}$ & 0.0035 & 0.0125 & P4-1 & 0.0164 & Beauveria bassiana & KT876586 \\
\hline $\begin{array}{l}\text { Oncology Unit } \\
\text { (W59) }\end{array}$ & & & P4-2 & 0.0084 & Gliomastix polychroma & KT876584 \\
\hline $\begin{array}{l}\text { Oncology Unit } \\
\text { (W60) }\end{array}$ & 0.0025 & 1.1746 & P5-1 & 0.8563 & Fusarium dimerum & KT876591 \\
\hline Oncology Unit & & & P5-2 & 0.8066 & Fusarium dimerum & KT876592 \\
\hline
\end{tabular}

40 
Fusarium-specific monoclonal antibody

\begin{tabular}{|c|c|c|c|c|c|c|}
\hline (W60) & & & & & & \\
\hline $\begin{array}{l}\text { Oncology Unit } \\
\text { (W60) }\end{array}$ & & & P5-3 & 0.0024 & Piptoporus betulinus & KT876609 \\
\hline $\begin{array}{l}\text { Oncology Unit } \\
\text { (W62) }\end{array}$ & 0.0011 & 0.0417 & P7-1 & 0.0097 & Trametes versicolor & KT876605 \\
\hline $\begin{array}{l}\text { Oncology Unit } \\
\text { (W63) }\end{array}$ & 0.0044 & 0.0060 & P8-1 & 0.0144 & Trametes versicolor & KT876606 \\
\hline $\begin{array}{l}\text { Oncology Unit } \\
\text { (W63) }\end{array}$ & & & P8-2 & 0.0106 & Stereum gausapatum & KT876601 \\
\hline $\begin{array}{l}\text { Oncology Unit } \\
\text { (W64) }\end{array}$ & 0.0022 & 0.0163 & P9-1 & 0.0101 & Stereum gausapatum & KT876600 \\
\hline $\begin{array}{l}\text { Oncology Unit } \\
\text { (W64) }\end{array}$ & & & P9-2 & 0.0068 & Trametes versicolor & KT876604 \\
\hline $\begin{array}{l}\text { Oncology Unit } \\
\text { (W64) }\end{array}$ & & & P9-3 & 0.0140 & Stereum gausapatum & KT876602 \\
\hline $\begin{array}{l}\text { Oncology Unit } \\
\text { (W65) }\end{array}$ & 0.0051 & 0.0280 & P10-1 & 0.0032 & $\begin{array}{l}\text { Phaeophlebiopsis } \\
\text { peniophoroides }\end{array}$ & KT876610 \\
\hline
\end{tabular}

1 a. S, sink; W, tap water from corresponding sink number; T, water sample from main tank.

2 b. Threshold absorbance value for detection in ELISA $\geq 0.100$. Shading indicates earliest point in 3 sampling process at which antigen was detectable in ELISA tests with mAb-ED7.

4 c. Fusarium strains in bold further characterized by TEF-1 $\alpha$ PCR analysis (Table $\underline{\mathrm{S} 3} 4$ and_-Supporting 5 pata Set 1Appendix 1).

6

7

8

9 Table 3. Summary of ELIS $\Lambda$ tests and mycological culture of sink swabs.

\begin{tabular}{|c|c|c|c|c|c|c|}
\hline $\begin{array}{l}\text { Total no. } \\
\text { sinks- } \\
\text { swabbed }\end{array}$ & $\begin{array}{l}\text { No. swab } \\
\text { samples } \\
\text { yielding } \\
\text { fungi } \\
(\%)^{a}\end{array}$ & $\begin{array}{c}\text { No. } \\
\text { stmples } \\
\text { positive } \\
\text { for } \\
\text { Fusarium } \\
\text { antigen at } \\
\text { swab- } \\
\text { stage }\end{array}$ & $\begin{array}{l}\text { No. samples } \\
\text { positive for } \\
\text { Fusarium } \\
\text { antigen by } \\
\text { mixed culture } \\
\text { stage }\end{array}$ & $\begin{array}{l}\text { No. samples- } \\
\text { positive for } \\
\text { Fusarium } \\
\text { antigen by } \\
\text { axenic culture- } \\
\text { stage- }\end{array}$ & $\begin{array}{l}\text { No. antigen- } \\
\text { positive- } \\
\text { samples } \\
\text { yielding } \\
\text { Fusarimm } \\
\text { spp. }\end{array}$ & $\begin{array}{l}\text { No. antigen- } \\
\text { positive- } \\
\text { samples not } \\
\text { yielding } \\
\text { Fusarium } \\
\text { spp. }\end{array}$ \\
\hline 65 & $\begin{array}{c}65 \\
(100 \%)\end{array}$ & $\begin{array}{c}34 \\
(52 \%)\end{array}$ & $\begin{array}{c}37 \\
(57 \%)\end{array}$ & $\begin{array}{c}54 \\
(83 \%)\end{array}$ & $\begin{array}{c}50 \\
(93 \%)\end{array}$ & $\begin{array}{c}4 \\
(7 \%)\end{array}$ \\
\hline
\end{tabular}

10

11 ta. Fustrium spp. and/or unrelated fungi.

12 
Fusarium-specific monoclonal antibody

1

2

3

4

5

6

7

8

9

10

11

12

13

14

15

16

17

18

19

20

21

22

Table 4. Translation Elongation Factor-1 $\alpha$ PCR analysis of Fusarium isolates recovered from sinks.

\begin{tabular}{|c|c|c|}
\hline Isolate No. & Location & TEF-1a ID \\
\hline A1 1 & University & F. oxysporum species complex 33 \\
\hline
\end{tabular}

42 


\section{Page 43 of 87}

Fusarium-specific monoclonal antibody

\begin{tabular}{|c|c|c|}
\hline A12 & University & F. oxysporum species complex 33 \\
\hline $\mathrm{A} 21$ & University & F. solani species complex $1-a$ \\
\hline A2-3 & University & F. oxysporum species complex 33 \\
\hline $\mathrm{A} 2-5$ & University & F. solani species complex 1-a \\
\hline A54 & University & F. oxysporum species complex 33 \\
\hline A5-6 & University & F. oxysporum species complex 33 \\
\hline A6-1 & University & F. oxysporum species complex 33 \\
\hline A6-2 & University & F. oxysporum species complex 33 \\
\hline A6-3 & University & F. oxysporum species complex 33 \\
\hline$A 6-4$ & University & F. solani species complex 9 a \\
\hline A7-3 & University & F. oxysporum species complex 183 \\
\hline A7-4 & University & F. oxysporum species complex 126 \\
\hline A84 & University & F. oxysporum species complex 33 \\
\hline 482 & University & F. oxysporum species complex 33 \\
\hline A9-1 & University & F. solani species complex 5-d \\
\hline A9-2 & University & F. solani species complex $5-\mathrm{d}$ \\
\hline A9-3 & University & F. solani species complex $1-c$ \\
\hline A9-4 & University & F. solani species complex $1-a$ \\
\hline B1-1 & University & F. dimerum species complex ET gr. \\
\hline B1-6 & University & F. dimerum species complex ET gr. \\
\hline B2-1 & University & F. dimerum species complex ET-gr. \\
\hline B2-5 & University & F. dimerum species complex ET gr. \\
\hline B4 1 & University & F. dimerum species complex ET gr. \\
\hline B5-1 & University & F. oxysporum species complex 16 \\
\hline B5-3 & University & F. oxysporum species complex 16 \\
\hline B6-1 & University & F. oxysporum species complex 16 \\
\hline B6-z & University & F. oxysporum species complex 16 \\
\hline B7-1 & University & F. oxysporum species complex 16 \\
\hline B7-6 & University & F. dimerum species complex ET-gr. \\
\hline B8 1 & University & F. solani species complex 15 a \\
\hline B9-2 & University & F. oxysporum species complex 99 \\
\hline B9-3 & University & F. solani species complex 1 a \\
\hline B10-1 & University & F. oxysporum species complex 33 \\
\hline B10-2 & University & F. oxysporum species complex 33 \\
\hline E1-3 & University & F. oxysporum species complex 33 \\
\hline $\mathrm{C} 1-4$ & University & F. dimerum species complex ET-gr. \\
\hline $\mathrm{C} 2-1$ & University & F. oxysporum species complex 33 \\
\hline E2-4 & University & F. oxysporum species complex 33 \\
\hline E27 & University & F. oxysporum species complex 33 \\
\hline E3-1 & University & F. oxysportm species complex 134 \\
\hline $\mathrm{C} 3-2$ & University & F. oxysporum species complex 134 \\
\hline C3-4 & University & F. oxysporum species complex 134 \\
\hline C4 1 & University & F. oxysporum species complex 33 \\
\hline C4-5 & University & F. dimerum species complex ET-gr. \\
\hline C5-1 & University & F. oxysporum species complex 33 \\
\hline 652 & University & F. oxysporum species complex 126 \\
\hline $65-3$ & University & F. oxysporam species complex 33 \\
\hline C5-4 & University & F. oxysporum species complex 33 \\
\hline C5-5 & University & F. oxysporum species complex 33 \\
\hline
\end{tabular}


Fusarium-specific monoclonal antibody

\begin{tabular}{|c|c|c|}
\hline $66-1$ & University & F. oxysporum species complex 33 \\
\hline $66-2$ & University & F. oxysporum species complex 33 \\
\hline C6-3 & University & F. oxysporum species complex 33 \\
\hline C6-4 & University & F. oxysportum species complex 33 \\
\hline $66-5$ & University & F. axysporum species complex 33 \\
\hline $67-1$ & University & F. oxysporum species complex 134 \\
\hline $68-1$ & University & F. oxysporum species complex 134 \\
\hline 682 & University & F. oxysporum species complex 134 \\
\hline 686 & University & F. oxysporum species complex 33 \\
\hline 691 & University & F. oxysporum species complex 134 \\
\hline C9-2 & University & F. oxysporum species complex 134 \\
\hline C9-3 & University & F. oxysporum species complex 134 \\
\hline 69-4 & University & F. oxysportm species complex 134 \\
\hline 69-5 & University & F. oxysporum species complex 134 \\
\hline $\mathrm{C10} 1$ & University & F. oxysporum species complex 114 \\
\hline $\mathrm{C} 10-2$ & University & F. solani species complex $2-V$ \\
\hline $\mathrm{C} 10-4$ & University & F. solani species complex $2-\forall$ \\
\hline 6107 & University & F. solani species complex 2 r \\
\hline CRT1-1 & University & F. oxysporum species complex 33 \\
\hline CRT1-2 & University & F. oxysporum species complex 33 \\
\hline ERT1 3 & University & F. oxysporum species complex 33 \\
\hline R1-1 & $\mathrm{ICU}$ & F. solani species complex 1 a \\
\hline R1-2 & $\mathrm{ICU}$ & F. solani species complex 1-a \\
\hline R1-3 & $\mathrm{ICU}$ & F. solani species complex 1-a \\
\hline R5 4 & ITU & F. dimerum species complex ET gr. \\
\hline R5-2 & ITU & F. dimerum species complex ET-gr. \\
\hline R5-3 & ITU & F. dimerum species complex ET gr. \\
\hline R6-1 & ITU & F. dimerum species complex ET gr. \\
\hline R6-2 & ITU & F. dimerum species complex ET-gr. \\
\hline R6-3 & ITU & F. dimerum species complex ET gr. \\
\hline R6-9 & ITU & F. solani species complex 20 d \\
\hline R7-1 & ITU & F. dimerum species complex ET-gr. \\
\hline R72 & ITU & F. dimerum species complex ET gr. \\
\hline R81 & ITU & F. dimerum species complex ET gr. \\
\hline R82 & ITU & F. dimerum species complex ET gr. \\
\hline R8-3 & ITU & F. dimerum species complex ET-gr. \\
\hline $\mathrm{X} 2-2$ & ITU & F. dimerum species complex ET-gr. \\
\hline$\times 2-4$ & ITU & F. dimerum species complex ET gr. \\
\hline $\mathrm{X} 2-5$ & ITU & F. dimerum species complex ET-gr. \\
\hline $\mathrm{X} 2-6$ & ITU & F. dimerum species complex ET-gr. \\
\hline $\mathrm{X} 34$ & Ophthalmelogy Unit & F. dimerum species complex ET gr. \\
\hline $\mathrm{X3-3}$ & Ophthalmology Unit & F. dimerum species complex ET gr. \\
\hline X4-1 & Ophthalmology Unit & F. dimerum species complex ET gr. \\
\hline $\mathrm{X} 4-2$ & Ophthalmology Unit & F. dimerum species complex ET-gr. \\
\hline $\mathrm{x} 5-2$ & Ophthalmology Unit & F. dimerum species complex ET-gr. \\
\hline$\times 5-4$ & Ophthalmology Unit & F. dimerum species complex ET gr. \\
\hline$K 23$ & Haematology Unit & F. solani species complex $5 \mathrm{k}$ \\
\hline K24 & Haematology Unit & F. solani species complex $5 \mathrm{k}$ \\
\hline K6-1 & Haematology Unit & F. oxysporum species complex 33 \\
\hline
\end{tabular}




\section{Page 45 of 87}

Fusarium-specific monoclonal antibody

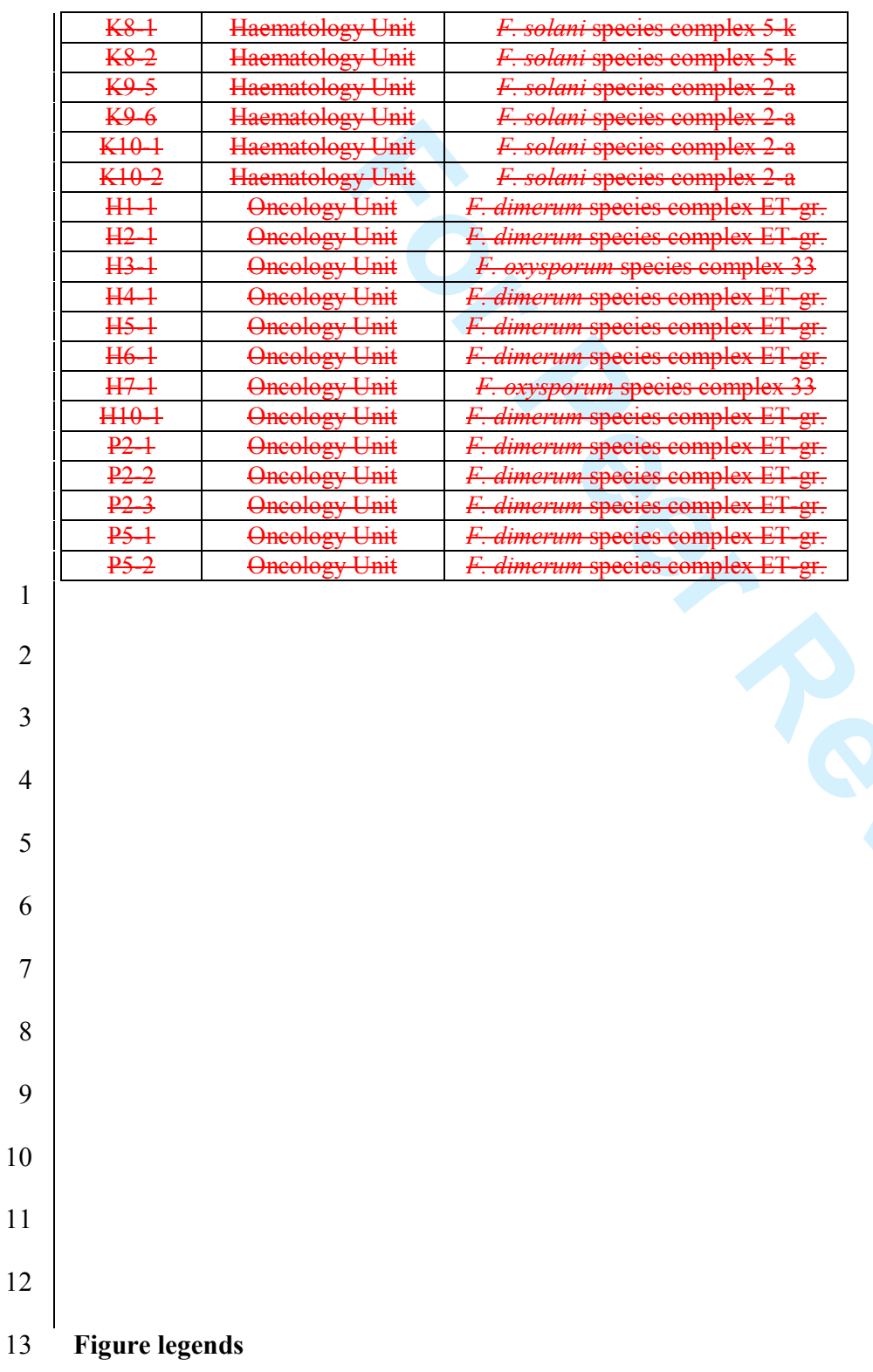


Fusarium-specific monoclonal antibody

1
Figure 1. Specificity of ED7 determined by Enzyme-Linked Immunosorbent Assay tests of surface washings containing water-soluble antigens from Fusarium species and related and unrelated yeasts and molds. (A) ELISA aELISA absorbance values at $450 \mathrm{~nm}$ for from specificity screening tests using $\mathrm{mAb}$ ED7 and antigens from $F$. solani and unrelated yeasts and mouldmolds $(\mathrm{A})$, and (B) for antigens from the $F$. solani teleomorph Haematonectria- haematococca and related Fusarium species-(B). Wells were coated with $60 \mu \mathrm{g}$ protein $\mathrm{ml}^{-1}$ buffer. Bars are the means of three biological replicates \pm standard errors and $-\underline{t}$ The threshold absorbance value for detection of antigen in ELISA is $\geq 0.100$ (indicated by lines on graphs). Numbers in parentheses after species names denote strain numbers with further details of strains provided in Table S1.

Figure 2. Characterisation Characterisation-of the ED7 antigen and its epitope and spatial distribution of the antigen in spores and hyphae-bound-by mAb_ED7. (A) Western immunoblot with $\mathrm{mAb}$-ED7 using culture fluid from 2-d-old PDB cultures of $F$. solani CBS224.34 (lane 1) and F. oxysporum f.sp. lycopersici CBS167.30 (lane 2). Wells were loaded with $1.6 \mu \mathrm{g}$ of protein. $\mathrm{M}_{\mathrm{t}}$ denotes molecular weight in $\mathrm{kDa}$. Note the major extracellular antigen with molecular weight of 200_kDa. (B) Absorbance values from ELISA tests with mAb-ED7 using immobilized antigensurface washings containing water-soluble antigens immobilized to the wells of microtitre plates and treated with trypsin or PBS only (control) at $4^{\circ} \mathrm{C}$ and $37^{\circ} \mathrm{C}$. Bars are the means of three biological replicates \pm standard errors and bars with the same letter are not significantly different at $\mathrm{p}<0.001$ (ANOVA and Tukey-Kramer test). (C) Absorbance values from ELISA tests with $-\mathrm{mAb}$ ED7 using surface washings containing water-soluble antigens immobilized to the wells of

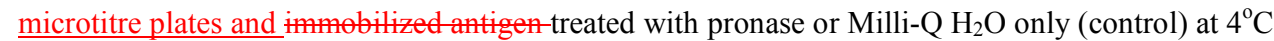
and $37^{\circ} \mathrm{C}$. Bars are the means of three biological replicates \pm standard errors and bars with the same
Formatted: Font: Bold

Formatted: Font: Italic

Formatted: Font: Not Bold

Formatted: Font: Not Bold

Formatted: Font: Bold

Formatted: Font: Bold

Formatted: Subscript 
Fusarium-specific monoclonal antibody

1

letter are not significantly different at $\mathrm{p}<0.001$ (ANOVA and Tukey-Kramer test). (D) Absorbance

values from ELISA tests with mAb ED7 following Stabilityheating of the water-soluble ED7 antigen following heating of surface washings antigen-at $100^{\circ} \mathrm{C}$ over a 70 min period. Treated antigen was subsequently immobilized to the wells of microtitre plates and assayed by ELISA. Bars are the means of three biological replicates \pm standard errors and bars with the same letter are not significantly different at $\mathrm{p}<0.001$ (ANOVA and Tukey-Kramer test). (E) Absorbance values from ELISA tests with ED7 using surface washings containing water-soluble antigens immobilized to the wells of microtitre plates and Absorbance values from ELISA tests with mAb ED7 and immobilized antigen treated with periodate (open circles) or with acetate only control (closed circles) at $4^{\circ} \mathrm{C}$ over a $20 \mathrm{~h}$ period. Each point is the mean of three biological replicates \pm standard errors. ( $\mathrm{F}$ to and $-\mathrm{I} G)$ Photomicrographs of $F$. solani CBS224.34 immunostained with mAb-ED7 or TCM control and goat anti-mouse polyvalent Ig fluorescein isothiocyanate (FITC) conjugate. (F) Brightfield image of germinated conidium with hypha probed with ED7 followed by fluorochrome conjugate (scale bar $=6 \mu \mathrm{m}) ;(\mathrm{G})$ Same field of view as panel F but examined under epifluorescence. Note intense staining of the cell wall of microconidium and hypha; $;$ Seale bar $=6 \mu \mathrm{m} .(\mathrm{H})$ Brightfield image of germinated conidium with hypha probed with TCM (negative control) followed by FITC conjugate (scale bar $=3 \mu \mathrm{m})$; (I) Same field of view as panel $\mathrm{H}$ but examined under epifluorescence. Note conidium incubated with $\mathrm{mAb}-\mathrm{ED} 7$ and anti-mouse immunoglobulin $20 \mathrm{~nm}$ gold particles, showing antigen in the cell wall and in an extracellular fimbrial matrix surrounding the spore (scale bar $=$ 
Fusarium-specific monoclonal antibody

1 (1)

matrix surrounding the cell $($ scale bar $=100 \mathrm{~nm})$; $(\underline{L} \underline{J})$ Transverse section of a microconidium incubated with TCMTCM ( (negative control) and anti-mouse immunoglobulin $20 \mathrm{~nm}$ gold particles, showing lack of staining by the secondary gold conjugatereperter. $\mathrm{S}$ (scale bar $=180 \mathrm{~nm}$. 
Fusarium-specific monoclonal antibody

1 Detection of human pathogenic Fusarium species in hospital and

2 communal sink biofilms by using a highly specific monoclonal

3 antibody

4

5 Marwan Al-Maqtoofi ${ }^{1,2}$ and Christopher R. Thornton ${ }^{1 *}$

$6 \quad{ }^{1}$ Biosciences, Geoffrey Pope Building, University of Exeter, Stocker Road, Exeter, EX4 4QD, UK.

$7 \quad{ }^{2}$ University of Basrah, College of Science, Biology Department, Basrah, Iraq.

8 *Corresponding author: Tel: +44 (0)1392 725172. Fax: +44 (0)1392 263434. E-mail address:

$9 \quad$ C.R.Thornton 0 ex.ac.uk

11 Summary

12 The fungus Fusarium is well known as a plant pathogen, but has recently emerged as an

13 opportunistic pathogen of humans. Habitats providing direct human exposure to infectious

14 propagules are largely unknown, but there is growing evidence that plumbing systems are sources

15 of human pathogenic strains in the Fusarium solani species complex (FSSC) and Fusarium

16 oxysporum species complex (FOSC), the most common groups infecting humans. Here, we use a

17 newly developed Fusarium-specific monoclonal antibody (mAb ED7) to track FSSC and FOSC

18 strains in sink drain biofilms by detecting its target antigen, an extracellular 200kDa carbohydrate,

19 in saline swabs. The antigen was detectable in $52 \%$ of swab samples collected from sinks across a

20 University campus and a tertiary care hospital. The mAb was $100 \%$ accurate in detecting FSSC,

21 FOSC and $F$. dimerum species complex (FDSC) strains that were present, as mixed fungal

22 communities, in $83 \%$ of sink drain biofilms. Specificity of the ELISA was confirmed by sequencing 
Fusarium-specific monoclonal antibody

1 of the internally transcribed spacer 1 (ITS1)-5.8S-ITS2 rRNA-encoding regions of culturable yeasts

2 and molds that were recovered using mycological culture, while translation elongation factor

3 (TEF)-1 $\alpha$ analysis of Fusarium isolates included FSSC 1-a, FOSC 33 and FDSC ET-gr, the most

4 common clinical pathotypes in each group.

5

\section{Originality-Significance Statement}

7 - Development of a monoclonal antibody (mAb ED7) specific to Fusarium, a fungal genus

$8 \quad$ containing human and plant pathogens

9 - Fusarium diagnostic antigen detected in swabs of sink drain biofilms, with 100\% accuracy

10 of mAb-based ELISA confirmed by ITS sequencing of mixed fungal communities

11 comprising human pathogenic yeasts and molds

12 - First report describing the use of a highly specifc mAb to track human pathogenic fusaria,

13 demonstrating widespread occurrence of pathogen in communal and hospital sinks with

14 potential for nosocomial and community acquired infections 
Fusarium-specific monoclonal antibody

\section{Introduction}

2 Species in the fungal genus Fusarium are ubiquitous environmental molds, and pathogens of both 3 plants and animals (Zhang et al., 2006; Thornton and Wills, 2015). In immunocompromised

4 humans, such as patients with haematological malignancies and hematopoietic stem cell and solid 5 organ transplant recipients, Fusarium species are significant emerging pathogens, causing a 6 frequently fatal disseminated disease known as fusariosis with an associated mortality rate of 50-

7 75\% (Girmenia et al., 2000; Musa et al., 2000; Boutati and Anaissie, 1997; Dignani and Anaissie, 8 2004; Jensen et al., 2004; Nucci and Anaissie, 2007). In some tertiary cancer centres, Fusarium has 9 emerged as the second most common mold pathogen after Aspergillus (Walsh and Groll, 1999;

10 Muhammed et al., 2011). Regardless of human immune status, Fusarium species can cause 11 localised nail infections (onychomycosis)(Arrese et al., 1996), bone and joint infections (Koehler et 12 al., 2014), infections of burn wounds (Latenser, 2003), skin infections (Nucci and Anaissie, 2002;

13 Gurusidappa and Mamatha, 2011), and are the most frequent cause of mycotic eye infections known 14 as fungal keratitis (Jurkunas et al., 2009), leading to progressive corneal destruction and 15 endophthalmitis, with loss of vision or even loss of the affected eye (Dursun et al., 2003; Edelstein 16 et al., 2012).

A recent multistate outbreak of fungal keratitis in the USA and in Singapore and Hong Kong

18 was associated with contact lens solution contaminated with multiple strains of Fusarium and which

19 led to visual loss in many patients and the need for corneal transplantation (Chang et al., 2006).

20 While such outbreaks are rare, disseminated Fusarium infections and keratomycoses have increased

21 in frequency over the past decade (Koehler et al., 2014) and an increasing body of evidence

22 suggests that the main environmental sources of human pathogenic Fusarium species are

23 contaminated water systems (Doggett, 2000; Anaissie et al., 2002; Anaissie et al., 2011; Mehl and 
Fusarium-specific monoclonal antibody

1 Epstein, 2008). A number of studies have recovered pathogenic Fusarium species from plumbing

2 fixtures and it is hypothesised that microbial biofilms on fixtures may serve as important reservoirs

3 of infectious Fusarium propagules in hospitals and homes (Mehl and Epstein, 2008; Short et al., 4 2011).

Identification of environmental reservoirs of human pathogenic molds including Fusarium

6 has typically relied on nucleic acid-based technologies following recovery of fungi using selective

7 media (Anaissie et al., 2002; Mehl and Epstein, 2008; Short et al., 2011; Anaissie et al., 2011;

8 Rougeron et al., 2014). Recently, highly specific monoclonal antibodies (mAb) have been used to

9 identify pathogenic species or species complexes in environmental samples containing mixed

10 populations of yeasts and molds (Thornton, 2009; Davies and Thornton, 2014; Thornton et al.,

11 2015). While mAb-based approaches similarly rely on culture for recovery of fungi from

12 environmental samples, detection of diagnostic antigens in crude culture extracts using genus- or

13 species-specific mAbs offers an attractive alternative approach to pathogen detection, particularly

14 when combined with unsophisticated diagnostic modalities such as lateral-flow technology

15 (Thornton, 2008; Thornton, 2012).

16 In this study, we set out to determine whether a newly developed Fusarium-specific mAb

17 (ED7) could be used to track the fungus by detecting a water-soluble diagnostic antigen in swabs of

18 communal and hospital sinks. By using the $\mathrm{mAb}$ in an enzyme-linked immunsorbent assay

19 (ELISA), we show that it can differentiate Fusarium species from other unrelated yeast and mold

20 pathogens of humans present in mixed fungal communities encountered in sink biofilms. The

21 ELISA represents a simple method for specific detection of Fusarium species in environmental

22 reservoirs and for identifying plumbing systems contaminated with the fungus. 
Fusarium-specific monoclonal antibody

\section{$1 \quad$ Results}

2 Production of hybridoma cell lines, isotyping of $m A b$ and specificity

3 A single fusion was performed and 389 hybridoma cell lines were screened for specificity against a

4 range of clinically relevant yeasts and molds (Table S1). The aim was to identify cell lines secreting

5 mAbs specific to Fusarium that could be used to track the fungus in environmental samples

6 containing mixed species of human pathogenic fungi. To this end, a single cell line, ED7, was

7 identified that produced mAb belonging to the immunoglobulin class $\mathrm{M}$ (IgM), which was genus-

8 specific, reacting in ELISA tests with antigens from Fusarium species and with the F. solani

9 teleomorph Haematonectria haematococca only (Figs. 1A and 1B). It did not cross-react with

10 antigens from a wide range of unrelated mold and yeast species (Fig. 1A).

12 Western blotting of the ED7 antigen and epitope characterization

13 Gel electrophoresis and western blotting studies showed that ED7 binds to a major antigen with

14 molecular weight of $\sim 200 \mathrm{kDa}$ which is secreted extracellularly by both $F$. solani and $F$. oxysporum

15 (Fig. 2A). Fusarium solani antigens were subjected to enzymatic (Fig. 2B and Fig. 2C), heat (Fig.

16 2D) and chemical (Fig. 2E) modifications in order to characterise the epitope bound by ED7.

17 Reductions in mAb binding following treatment with pronase shows that its epitope consists of

18 protein, while reductions with trypsin indicate a protein epitope containing positively charged lysine

19 and arginine side chains. The lack of reduction in ED7 binding following digestion of immobilized

20 antigen with trypsin (Fig. 2B) and pronase (Fig. 2C) shows that it does not bind to a protein epitope.

21 Reductions in $\mathrm{mAb}$ binding following heat treatment shows that an epitope is heat labile. There was

22 no significant reduction in ED7 binding over 70 min of heating, showing that its epitope is heat

23 stable (Fig. 2D). Reductions in mAb binding following chemical digestion of an antigen with 
Fusarium-specific monoclonal antibody

1 periodate shows that its epitope is carbohydrate and contains vicinal hydroxyl groups. The

2 pronounced reductions in ED7 binding following periodate oxidation shows that its epitope consists

3 of carbohydrate residues (Fig. 2E). Taken together, these results indicate that ED7 binds to an

4 extracellular antigen and that its epitope is a heat stable carbohydrate moeity containing vicinal

5 hydroxyl groups.

7 Immunofluorescence and immunogold electron microscopy

8 Immuno-localisation studies using IF showed that the ED7 antigen was present on the surface of 9 spores and hyphae (Figs. 2F-I), while IEM showed that the antigen was present in the spore and

10 hyphal cell wall and in an extracellular fibrillar matrix surrounding both (Figs. 2J-L). In the TEM

11 image shown in Fig. 2J, 56\% of gold particles were distributed in the fibrillar matrix surrounding 12 the cell, while $40 \%$ and $4 \%$ of gold particles were distributed in the cell wall and cytoplasm 13 respectively. This shows that the ED7 antigen is predominantly extracellular or located within the 14 cell wall.

16 Immunodetection of Fusarium species in sink swabs and identification of fungi by analysis of the

17 ITS regions of the rRNA-encoding gene unit and by Translation Elongation Factor-1 $\alpha$ PCR

18 Monoclonal antibody ED7 was highly specific for the three human pathogenic species of Fusarium, 19 F. solani, F. oxysporum and F. dimerum, which were culturable from $75 \%$ of the sink swabs (Table

201 and Table S2)). ELISA tests of the saline sink swabs showed that $52 \%$ contained detectable levels

21 of Fusarium antigen (Table 1 and Table S2), with ELISA absorbance values in the range $\geq 0.100$

22 (the threshold value for antigen detection) and up to 1.500. In four hospital samples (samples S47,

23 S48 and S49 from ophthalmology and sample S64 from oncology) Fusarium strains could not be 
Fusarium-specific monoclonal antibody

1 recovered for identification by ITS sequencing despite detection of the diagnostic antigen in swab

2 samples with absorbance values of $0.264,0.530,0.187$ and 0.193 respectively (Table 1 ). This was

3 likely due to the Fusarium isolates being outgrown in the mixed culture plates by faster growing or

4 more abundant unrelated fungi. Importantly, ED7 was shown not to cross-react with unrelated fungi

5 (axenic culture absorbance values of $\leq 0.100$ in all cases) including the human pathogenic yeast or

6 yeast-like fungi Candida, Exophiala, Meyerozyma, Rhodotorula, Trichosporon, the human

7 pathogenic hyaline or dematiaceous molds Aspergillus, Phialophora, Phoma, Trichoderma, and the

8 human pathogenic mucormycete Mucor (Table 1). The remaining 93\% of samples positive for

9 Fusarium antigen, either at the swab stage or following periods of biological amplification in mixed

10 or axenic cultures (Table S2), yielded strains of the three Fusarium species. There was $100 \%$

11 concordance between Fusarium genus identification by ELISA and species identification by ITS

12 sequencing (Table 1). The species of $F$. solani and $F$. oxysporum recovered from sink swabs were

13 subsequently shown by TEF-1 $\alpha$ PCR (Supporting Data Set 1) to belong to F. solani species

14 complex (FSSC) 1-a, 1-c, 2-a, 2-v, 5-d, 5-k, 9-a, 15-a, 20-d and F. oxysporum (FOSC) species

15 complexes 16, 33, 99, 111, 126, 134, 183 (Table S3). All of the recovered $F$. dimerum isolates

16 belonged to the $F$. dimerum species complex (FDSC) ET-gr (Table S3). ITS analysis of axenic

17 cultures (Table 1) showed that a number of sink samples (e.g. S2, S6, S17, S19, S21, S24, S30,

18 S38) contained mixtures of Fusarium species, while ITS and TEF-PCR analysis (Table 1 and Table

19 S3) showed that others contained mixtures of species complexes of the same species (e.g. S8, S9,

20 S25, S28). Monoclonal antibody ED7 was able to detect all of the Fusarium species complexes

21 recovered in this study.

In addition to drain swabs, water samples were collected from the taps of sinks in the 23 hospital haematology and oncology units and from the main water tanks feeding the ophthalmology 
Fusarium-specific monoclonal antibody

1 unit. The ED7 diagnostic antigen could not be detected in any of the water samples directly and,

2 while all of the samples yielded fungi, only two of the tap samples (oncology W57 and W60)

3 contained Fusarium strains that belonged to FDSC ET-gr. and which were detectable by ELISA at

4 the mixed culture stage (Table 1). The sink biofilms corresponding to these water samples were also

5 positive at the swab ELISA stage (Table 1).

6

7

8

9

10

11

12

13

14

15

16

17

18

19

20

21

22

23

Wiley-Blackwell and Society for Applied Microbiology 
Fusarium-specific monoclonal antibody

\section{Discussion}

2 The genus Fusarium comprises ubiquitous environmental molds capable of infecting plants and

3 humans (Zhang et al., 2006). Unlike agriculture, where the most economically damaging pathogens

4 are considered to be F. graminearum and F. oxysporum (Dean et al., 2012), the species most

5 commonly cited as human pathogens belong to the Fusarium solani species complex (FSSC,

6 responsible for $50 \%$ of reported infections in humans), followed by strains in the Fusarium

7 oxysporum species complex (FOSC)(Torres and Kontoyiannis, 2011). The Fusarium dimerum

8 species complex (FDSC) is less frequently reported as causing human disease, but it is similarly

9 capable of causing disseminated infections in immunocompromised patients (Bigley et al., 2004;

10 Schroers et al., 2009).

11 While the natural habitats of plant pathogenic Fusarium strains are well characterised as soil

12 and decaying plant material, habitats providing direct human exposure to infectious propagules are

13 largely unexplored. The increasing frequency of opportunistic fungal infections in humans means

14 that improved surveillance methods are needed to identify environmental reservoirs of pathogens to

15 limit the exposure of vulnerable individuals to potentially infective propagules. For Fusarium, there

16 is a growing body of evidence to suggest that domestic and municipal water systems are potential

17 reservoirs of human pathogenic strains in the FSSC, FOSC and FDSC groups (Short et al., 2011).

18 Accurate techniques that can be used to identify the fungus in environmental samples

19 containing mixed populations of fungi are currently lacking and, while nucleic acid-based

20 technologies have been developed for the differentiation of Fusarium from other human pathogenic

21 species and to identify Fusarium species complexes, such techniques have typically been used in

22 retrospective analysis of axenic cultures collected during human and environmental population

23 studies (Bouchara et al., 2009; Steinmann et al., 2011; Lackner et al., 2012). Furthermore, these 
Fusarium-specific monoclonal antibody

1 studies have often employed Fusarium-selective media that eliminate other fungi present in

2 polymicrobial communities (Short et al., 2011). While monoclonal antibodies (mAbs) and antibody

3 fragments have been developed for detecting and differentiating Fusarium species in vitro or in

4 planta (Wong et al., 1988; Arie et al., 1991, 1995; Danks et al., 1996; Hayashi et al., 1998; Hu et

5 al., 2012, 2013), no attempts have been made to use mAbs to track human pathogenic strains in

6 environmental samples. Jensen et al. (2011) recently reported the development of Fusarium-

7 specific mAbs for immunohistochemical diagnosis of fusariosis. The IgM mAbs, which recognise

851 and $63 \mathrm{kDa}$ antigens, reacted strongly with fungal elements in both experimentally infected

9 animals and biopsy samples from patients with fusariosis sepsis and dissemination to the skin.

10 In this prospective study, we set out to determine whether human pathogenic species of

11 Fusarium could be identified in sink drains directly by using crude antigen extracts of biofilms and

12 detection using a genus-specific immunoglobulin $\mathrm{M}(\operatorname{IgM}) \mathrm{mAb}$, ED7, that binds to an extracellular

$13 \sim 200 \mathrm{kDa}$ carbohydrate antigen present on the surface of spores and hyphae. While the function of

14 the antigen is currently unknown we were able, in Enzyme-Linked Immunosorbent Assay (ELISA)

15 tests, to detect its presence in $52 \%$ of swab samples and, following biological amplification of

16 biofilms on a non-selective mycological medium, were able to identify additional biofilm samples

17 containing pathogenic strains of Fusarium. This is the first time, to our knowledge, that a mAb-

18 based detection method has been used to track Fusarium in environmental samples. The mAb was

19 able to differentiate Fusarium from a wide spectrum of unrelated fungi, including the human

20 pathogens Aspergillus (Thornton and Wills, 2015), Candida, Geotrichum, Rhodotorula and

21 Trichosporon (Davies and Thornton, 2014; Miceli et al., 2011), Cyphellophora and Phialophora

22 (Feng et al., 2014), Exophiala (Zeng et al., 2007), Trichoderma (Sandoval-Denis et al., 2014),

23 Engyodontium (Macêdo et al., 2007; Thamke et al., 2015) and Mucor (Petrikkos et al., 2012), 
Fusarium-specific monoclonal antibody

1 several of which have been reported previously to inhabit biofilms in water distribution systems

2 (Dogget, 2000). The 100\% accuracy of the ED7 ELISA, confirmed by using ITS sequencing and

3 TEF PCR analysis of recovered isolates, demonstrates its robustness in detecting potentially

4 infectious Fusarium species in polymicrobial communities. Importantly, ED7 reacted with all of the

5 species complex strains isolated including the most common clinical pathotypes of Fusarium, FSSC

6 1-a, FOSC 33 and FDSC ET-gr (Schroers et al., 2009; Short et al., 2011).

7 While the ED7 ELISA was able to identify Fusarium to the level of genus only, the

8 simplicity of the mAb-based approach to detection, even when combined with a standard

9 mycological isolation procedure, means that a recognised environmental niche of this group of

10 pathogenic fungi can be monitored readily. The widespread occurrence of human pathogenic

11 Fusarium species in sinks of a tertiary care hospital and sinks of a heavily populated university

12 campus, show that indoor plumbing-associated biofilms and water sources are an unseen source of

13 Fusarium infectious propagules for nosocomial and community-acquired infections of vulnerable

14 individuals, an observation consistent with previous studies (Annaisie et al., 2011; Short et al.,

15 2011). While no cases of fusariosis were reported during the course of this study, the close

16 proximity of the patients to hospital sinks colonised with both pathogenic fusaria and with other

17 opportunistic fungal pathogens is a serious concern given the known vulnerability of

18 immunocompromised individuals to invasive fungal infections.

\section{Acknowledgements}

21 M. Al-Maqtoofi was funded by a Ministry of Higher Education and Scientific Research (MOHESR)

22 of Iraq studentship (No. S634), to whom we are grateful. The authors would also like to thank the

23 RD\&E hospital for allowing us to sample patient sinks. 
Fusarium-specific monoclonal antibody

\section{Conflicts of Interest}

2 We declare that none of the authors involved in writing this paper have any conflicts of interest with 3 respect to the content of this article.

4

5

6

7

8 
Fusarium-specific monoclonal antibody

\section{Experimental procedures}

\section{Ethics statement}

4 All animal work described in this study was conducted under a UK Home Office Project License, 5 and was reviewed by the institution's Animal Welfare Ethical Review Board (AWERB) for

6 approval. The work was carried out in accordance with The Animals (Scientific Procedures) Act

71986 Directive 2010/63/EU, and followed all the Codes of Practice which reinforce this law,

8 including all elements of housing, care, and euthanasia of the animals. Permission for sink sampling

9 at the Royal Devon and Exeter Hospital was granted by the Director of Infection Prevention and 10 Control.

12 Fungal culture

13 Fungi (Table S1) were routinely cultured on Potato Dextrose Agar (PDA: 70139; Sigma), 14 Sabouraud Dextrose Agar (SDA: Sabouraud Dextrose Broth (SDB: S3306; Sigma) containing 2\% 15 (w/v) agar), Malt Yeast extract Agar (MYA: Y3127; Sigma), or Oatmeal Agar (OA: O3506; 16 Sigma), sterilized by autoclaving at $121^{\circ} \mathrm{C}$ for $15 \mathrm{~min}$. Cultures were grown at $26^{\circ} \mathrm{C}$ under a $16 \mathrm{~h}$ 17 fluorescent light regime.

19 Development of $m A b$, preparation of immunogen, and immunisation regime

$20 \mathrm{BALB} / \mathrm{c}$ mice were immunized with soluble antigens prepared from lyophilized mycelium of a 21 human pathogenic strain of Fusarium solani species complex 1-a (CBS strain 224.34). Conidia

22 were suspended in water after 10-day old PDA slant cultures were flooded with $5 \mathrm{ml} \mathrm{dH}_{2} \mathrm{O}$ and 23 gently agitated with an inoculation loop. Conidial suspensions were then filtered through Miracloth 
Fusarium-specific monoclonal antibody

1 to remove mycelium and transferred to $1.5 \mathrm{ml}$ micro-centrifuge tubes. The conidia were washed

2 three times with $\mathrm{dH}_{2} \mathrm{O}$ by repeated vortexing and centrifugation at 14,462 $\mathrm{g}$ for 5 min and finally

3 suspended in $\mathrm{dH}_{2} \mathrm{O}$ to give a concentration of $10^{6}$ conidia $\mathrm{ml}^{-1}$ solution. Flasks containing $100 \mathrm{ml}$ of

4 sterilized Potato Dextrose Broth (PDB: P6685; Sigma) were inoculated with $200 \mu$ of the conidial

5 suspension and incubated with shaking $(75 \mathrm{rpm})$ for $48 \mathrm{~h}$ at $26^{\circ} \mathrm{C}$. Hyphal biomass was collected on

6 Miracloth, snap frozen in liquid $\mathrm{N}_{2}$, and lyophilized. Culture filtrates were retained for gel

7 electrophoresis and western blotting studies and stored at $-20^{\circ} \mathrm{C}$ until required. One $\mathrm{mg}$ of

8 lyophilized biomass was suspended in $1 \mathrm{ml}$ phosphate buffered saline (PBS: $0.8 \% \mathrm{NaCl} ; 0.02 \%$

$\left.9 \mathrm{KCl} ; 0.115 \% \mathrm{Na}_{2} \mathrm{HPO}_{4} ; 0.02 \% \mathrm{KH}_{2} \mathrm{PO}_{4} ; \mathrm{pH} 7.2\right)$ and the resultant suspension centrifuged for $5 \mathrm{~min}$

10 at $14,462 \mathrm{~g}$. The supernatant, containing solubilized antigens, was used as the immunogen and as a

11 source of antigens for hybridoma screening assays. For immunization, 6-wk-old BALB/c female

12 white mice were given four intraperitoneal injections (300 $\mu$ l per injection) of antigen extract

13 containing $2.3 \mathrm{mg}$ protein $\mathrm{ml}^{-1}$ PBS at 2-wk intervals and a single booster injection five days before

14 fusion.

\section{Production and screening of hybridomas and determination of antibody specificity}

17 Hybridoma cells were produced by the method described elsewhere (Thornton, 2001) and the 18 supernatants were screened by Enzyme-Linked Immunosorbent Assay (ELISA) against antigens

19 immobilized to the wells of Maxisorp microtitre plates (442404; Nunc)(50 $\mu$ l per well). For

20 antibody specificity tests, fungi were grown on replicate agar slopes and surface washings

21 containing water-soluble antigens prepared as described in Thornton (2001). Protein concentrations,

22 determined spectrophotometrically at $280 \mathrm{~nm}$ (Nanodrop, Agilent Technologies Limited, Berkshire,

$23 \mathrm{UK}$ ), were adjusted to $60 \mu \mathrm{g} \mathrm{ml}^{-1}$ buffer. Fifty $\mu \mathrm{l}$ volumes were then used to coat the wells of 
Fusarium-specific monoclonal antibody

1 microtitre plates. After incubating overnight at $4^{\circ} \mathrm{C}$, wells were washed four times with PBST (PBS

2 containing Tween-20, 0.05\% (v/v)), once each with $\mathrm{PBS}$ and $\mathrm{dH}_{2} \mathrm{O}$ and then air-dried at $23^{\circ} \mathrm{C}$ in a

3 laminar flow hood. The plates were stored in sealed plastic bags at $4^{\circ} \mathrm{C}$ in preparation for screening

4 of hybridoma supernatants by ELISA as described below.

6 Enzyme-Linked Immunosorbent Assay

7 Wells containing immobilized antigens were incubated successively with hybridoma tissue culture

8 supernatant (TCS) for $1 \mathrm{~h}$, followed with goat anti-mouse polyvalent (immunoglobulin classes IgG, 9 IgA, and IgM) peroxidase conjugate (A-0412; Sigma Chemical Company, Poole, United Kingdom)

10 diluted 1 in 1000 in PBST for a further hour. Bound antibody was visualized by incubating wells

11 with tetramethyl benzidine (TMB: T-2885; Sigma) substrate solution (Thornton, 2001) for 30 min.

12 The reactions were stopped by the addition of $3 \mathrm{M} \mathrm{H}_{2} \mathrm{SO}_{4}$ and absorbance values were determined

13 at $450 \mathrm{~nm}$ with an MRX automated microplate reader (Dynex Technologies, Billingshurst, UK).

14 Wells were given four 5-min rinses with PBST between incubations and a final rinse with PBS

15 before addition of the substrate solution. Working volumes were $50 \mu \mathrm{l}$ per well and control wells

16 were incubated with tissue culture medium (TCM) containing $10 \%(\mathrm{v} / \mathrm{v})$ fetal bovine serum. All

17 incubation steps were performed at $23^{\circ} \mathrm{C}$ in sealed plastic bags. The threshold for detection of the

18 antigen in ELISA was determined from control means ( 2 x TCM absorbance values)(Sutula et al.,

19 1986). These values were consistently in the range $0.050-0.100$. Consequently, absorbance values

$20>0.100$ were considered as positive for the detection of antigen. 
Fusarium-specific monoclonal antibody

2 The Ig class of mAbs was determined by using antigen-mediated ELISA. Wells of microtitre plates

\section{Determination of Ig subclass and cloning procedure}

3 coated with $F$. solani CBS224.34 water-soluble antigens from surface washings were incubated

successively with ED7 TCS for $1 \mathrm{~h}$, followed with goat anti-mouse $\operatorname{IgG}_{1}, \operatorname{IgG}_{2 \mathrm{a}}, \operatorname{IgG}_{2 \mathrm{~b}}, \operatorname{IgG}_{3}, \operatorname{IgM}$, or IgA-specific antiserum (ISO-2; Sigma) diluted 1 in 3000 in PBST for 30 min and rabbit anti-goat peroxidase conjugate diluted 1 in 1000 (A-5420; Sigma) for a further 30 min. Bound antibody was visualized with TMB substrate as described above. Hybridoma cells lines were sub-cloned three times by limiting dilution, and cell lines were grown in bulk in a non-selective medium preserved by slowly freezing in fetal bovine serum/dimethyl sulfoxide (92:8 [v/v]), and stored in liquid nitrogen.

\section{Gel electrophoresis and Western blotting}

For sodium-dodecyl-sulphate-polyacrylamide gel electrophoresis (SDS-PAGE), culture filtrates from 2-d-old PDB shake cultures of F. solani CBS224.34 and F. oxysporum f.sp. lycopersici CBS167.30, prepared as described, were diluted in Laemmli buffer (Laemmli, 1970) and were denatured by heating at $95^{\circ} \mathrm{C}$ for $10 \mathrm{~min}$. Antigens were separated in $4-20 \%(\mathrm{w} / \mathrm{v})$ polyacrylamide gradient gels (161-1159; Bio-Rad) for $1.5 \mathrm{~h}$ at $23^{\circ} \mathrm{C}(165 \mathrm{~V})$ under denaturing conditions, and prestained broad range markers (161-0318; Bio-Rad) were used for molecular weight determinations. For westerns, separated antigens were transferred electrophoretically to a PVDF membrane (1620175; Bio-Rad). The membranes were blocked for $16 \mathrm{~h}$ at $4^{\circ} \mathrm{C}$ with PBS containing $1 \%(\mathrm{w} / \mathrm{v})$ bovine serum albumin (BSA) and incubated with ED7 TCS diluted 1 in 2 with PBS containing $0.5 \%(\mathrm{w} / \mathrm{v}) \mathrm{BSA}(\mathrm{PBSA})$ for $2 \mathrm{~h}$ at $23^{\circ} \mathrm{C}$. After washing three times with PBS, membranes were incubated for $1 \mathrm{~h}$ with goat anti-mouse IgM ( $\mu$-chain specific) alkaline phosphatase conjugate (A- 
Fusarium-specific monoclonal antibody

1 9688; Sigma), diluted 1 in 15,000 in PBSA. After the membranes were washed twice with PBS and

2 once with PBST, the bound antibodies were visualized by incubation in BCIP/NBT substrate

3 solution. Reactions were stopped by immersion in $\mathrm{dH}_{2} \mathrm{O}$ and air-dried between sheets of Whatman

4 filter paper.

5

6 Characterization of antigen by enzymatic and chemical modifications and by heating

7 Water-soluble antigens from surface washings of slopes of F. solani CBS224.34 were prepared as

8 described. Heat stability studies were conducted by placing tubes of solubilised antigen in a boiling

9 water bath. At $10 \mathrm{~min}$ intervals, samples were removed, centrifuged at 14,462 $\mathrm{g}$ for $5 \mathrm{~min}$, and

10 antigens immobilised to the wells of microtitre plates for assay by ELISA as described. For

11 periodate oxidation, microtitre wells containing immobilised antigens from surface washings of the

12 fungus were incubated with $50 \mu \mathrm{l}$ of sodium meta-periodate solution $\left(20 \mathrm{mM} \mathrm{NaIO}_{4}\right.$ in $50 \mathrm{mM}$

13 sodium acetate buffer ( $\mathrm{pH} 4.5)$ ) or acetate buffer only (control) at $4^{\circ} \mathrm{C}$ in sealed plastic bags. Plates

14 were given four 3-min PBS washes before processing by ELISA as described. For protease

15 digestions, microtitre wells containing immobilised antigen were incubated with $50 \mu 1$ of pronase

16 (protease XIV; $9 \mathrm{mg} \mathrm{ml}^{-1}$ in PBS) or trypsin $\left(1 \mathrm{mg} \mathrm{ml}^{-1}\right.$ in Milli-Q $\left.\mathrm{H}_{2} \mathrm{O}\right)$ solution or Milli-Q $\mathrm{H}_{2} \mathrm{O}$ or

17 PBS only controls respectively for $4 \mathrm{~h}$ at $37^{\circ} \mathrm{C}$ or $4^{\circ} \mathrm{C}$. Plates were given four 3 -min rinses with

18 PBS and then assayed by ELISA with ED7 TCS as described.

\section{Immunofluorescence and immunogold electron microscopy}

21 For immunfluorescence (IF), sterilised slides were coated with a washed spore suspensions of $F$.

22 solani CBS224.34 containing $1 \%(\mathrm{w} / \mathrm{v})$ glucose solution and incubated at $26^{\circ} \mathrm{C}$ for $16 \mathrm{~h}$ to allow

23 spore germination and formation of germ tubes. After air-drying, the cells were fixed to the slides as 
Fusarium-specific monoclonal antibody

1 described in Thornton (2001) and incubated with ED7 TCS or TCM only (negative control) for $1 \mathrm{~h}$,

2 followed by three 5 min PBS washes. Slides were then incubated with goat anti-mouse polyvalent

3 fluorescein isothiocyanate (FITC) conjugate (diluted 1 in 40 in PBS)(F1010; Sigma) for 30 min.

4 Slides were given three 5 min washes with PBS and mounted in PBS-glycerol mounting medium

5 (F4680; Sigma) before overlaying with coverslips. All incubation steps were performed at $23^{\circ} \mathrm{C}$ in a

6 humid environment to prevent evaporation and slides were stored in the dark, at $4^{\circ} \mathrm{C}$, prior to

7 examination using an epifluorescence microscope (Olympus IX81) fitted with $495 \mathrm{~nm}$ (excitation)

8 and $518 \mathrm{~nm}$ (emission) filters for FITC. For immunogold electron microscopy (IEM) the method

9 described in Thornton \& Talbot (2001) was used. Spores and hyphae of F. solani were prepared by

10 incubating washed conidia in $1 \%(\mathrm{w} / \mathrm{v})$ glucose solution at $26^{\circ} \mathrm{C}$ for $16 \mathrm{~h}$ to allow spore

11 germination and formation of germ tubes. Cells were embedded in LR White resin (Agar Scientific

12 Ltd.) and ultra thin sections prepared for immunolabeling. Sections immobilized to nickel grids

13 were blocked by immersion in PBST containing 1\%(w/v) BSA (PBST-BSA) which had been

14 sterile filtered through a $0.2 \mu \mathrm{m}$ filter. The grids were washed three times ( 3 min each) in sterile

15 filtered PBST and then incubated in ED7 TCS or TCM only (negative control) for $1 \mathrm{~h}$. After four

16 washes (3 min each) with sterile filtered PBST, the grids were incubated for a further hour in PBST-

17 BSA containing a 1:20 dilution of goat anti-mouse $20 \mathrm{~nm}$ gold conjugate (EM.GAF20; BBI

18 Solutions). The grids were washed four times (3 min each) in sterile filtered PBST and then placed

19 on Whatman filter paper to dry. Dried grids were then incubated for $20 \mathrm{~min}$ in $2 \%(\mathrm{w} / \mathrm{v})$ uranyl

20 acetate solution followed by $2 \%(\mathrm{w} / \mathrm{v})$ lead citrate solution for $4 \mathrm{~min}$. Working volumes were $100 \mu \mathrm{l}$

21 and incubation and washing steps were carried out at at $23^{\circ} \mathrm{C}$. Immunostained samples were

22 examined using a Jeol JEM 1400 transmission electron microscope fitted with a Gatan ES 100W

23 CCD camera.

Wiley-Blackwell and Society for Applied Microbiology 
Fusarium-specific monoclonal antibody

\section{$1 \quad$ Statistical analysis}

2 Unless otherwise stated, numerical data were analysed using the statistical programme Minitab

3 (Minitab 16, Minitab®, Coventry, UK). Analysis of variance (ANOVA) was used to compare

4 means of more than two data sets and Post-hoc Tukey-Kramer analysis was then performed to 5 distinguish which sets were significantly different from one another.

\section{$7 \quad$ Sampling from drains}

8 A total of 65 sinks were swabbed, comprising 32 sinks across the ICU, ITU, haematology, oncology

9 and ophthalmology units of the Royal Devon and Exeter tertiary care hospital (Exeter, Devon, UK)

10 and 33 restroom sinks located around the University of Exeter campus (Exeter, Devon, UK). In

11 addition, cold-water samples were collected from taps connected to the sinks in the haematology

12 and oncology unit, and from the two main water tanks feeding the ophthalmology unit. To isolate

13 fungi from sink biofilms, sterile cotton buds (Boots, UK) wetted with PBS were used to scour the

14 inner surfaces of sink drainpipes for approximately $10 \mathrm{~s}$. Swabs with visible detritus were immersed

15 in 1.5-ml micro-centrifuge tubes containing $1 \mathrm{ml}$ PBS to dislodge biofilm debris, and the sealed

16 tubes transferred to the laboratory for processing by ELISA and mycological culture.

18 Immunodetection of Fusarium species in sink swabs and identification of fungi by analysis of the

19 ITS regions of the rRNA-encoding gene unit and Translation Elongation Factor-1 $\alpha$ PCR

20 Biofilm debris was pelleted by centrifugation at $14,462 \mathrm{~g}$ for $5 \mathrm{~min}$ and $50 \mu \mathrm{l}$ samples of 21 supernatant transferred to the wells of microtitre plates for assay by ELISA (Table 1 and Table S2;

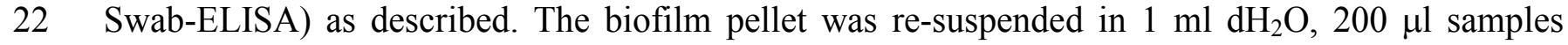
23 spread on the surface of PDA containing $1 \mu \mathrm{g} \mathrm{ml}^{-1}$ of the broad-spectrum antibiotic rifampicin, and 
Fusarium-specific monoclonal antibody

1 the plates incubated for $2 \mathrm{~d}$ at $26^{\circ} \mathrm{C}$ under a $16 \mathrm{~h}$ fluorescent light regime. Fungi in these mixed

2 culture plates were separated on the basis of gross morphological characteristics and axenic slope

3 cultures generated following sub-culture on PDA. Crude antigen extracts were prepared as surface

4 washings from mixed cultures and from axenic cultures and assayed by ELISA (Table 1 and Table

5 S2; Mixed culture-ELISA and Axenic culture-ELISA, respectively) as described.

$6 \quad$ Fungal DNA was extracted from axenic culture material by using the CTAB method (Chow

$7 \&$ Kafer, 1993) and fungi were identified by sequencing of the ITS1-5.8S-ITS2 region of the rRNA-

8 encoding gene unit (White et al., 1990) according to procedures described elsewhere (Thornton et

9 al., 2002), using the primers ITS1ext (5'-GTAACAAGGTTTCCGTAGGTG-3') and ITS4ext (5'-

10 TTCTTTTCCTCCGCTTATTGATATGC-3'). Species identity was predicted based on $>95 \%$

11 sequence identity $(\mathrm{E}-\mathrm{value}=0.0)($ Altschul et al. 1997) of the ITS1-5.8S-ITS2 region of recovered

12 species to species recorded in GenBank. Fusarium species were further identified to species

13 complex level by using the forward primer ef-1 (5'-ATGGGTAAGGA(A/G)GACAAGAC-3') and

14 reverse primer ef-2 (5'-GGA(G/A)GTACCAGT(G/C)ATCATGTT-3'), which amplify an 700 bp

15 region of Translation Elongation Factor 1-alpha (TEF-1 $\alpha$ ), the single-locus identification tool in

16 Fusarium (Geiser et al., 2004). PCR reactions were carried out in a total volume of $25 \mu 1$ consisting

17 of $1 \mu \mathrm{l}$ DNA at a concentration of $30-75 \mathrm{ng} \mu \mathrm{l}^{-1}, 12.5 \mu \mathrm{l}$ of GoTaq ${ }^{\circledR}$ Green Master Mix DNA

18 polymerase (Promega, MF7112), $9.5 \mu \mathrm{l}$ of nuclease free water (Promega) and $1 \mu \mathrm{l}$ of each primer at

1920 pmol. The following cycling parameters were used: an initial denaturation step at $95^{\circ} \mathrm{C}$ for 8

20 min; 35 cycles of $15 \mathrm{sec}$ at $95^{\circ} \mathrm{C}$ (denaturation); $20 \mathrm{~s}$ at $54^{\circ} \mathrm{C}$ (annealing), $1 \mathrm{~min}$ at $72^{\circ} \mathrm{C}$ (extension)

21 followed by a final $5 \mathrm{~min}$ extension step at $72^{\circ} \mathrm{C}$. Phylogenetic sub-groups of Fusarium species

22 were determined by interrogation of the FUSARIUM-ID v. 1.0 database

23 (http://isolate.fusariumdb.org)(O’Donnell et al., 2010), with the newly acquired TEF-1 $\alpha$ sequences 
Fusarium-specific monoclonal antibody

1 (Supporting Data Set 1).

2

$3 \quad$ Nucleotide sequence accession numbers

4 Newly determined ITS sequences were submitted to GenBank and the ITS accession numbers

5 KT876496 to KT876723 were obtained. Species designations of recovered fungi are shown in Table

61.


Fusarium-specific monoclonal antibody

\section{References}

2 Altschul, S.F., Madden, T.L., Schaffer, A.A., Zhang, Z., Miller, W., and Lipman, D.J. (1997)

3 Gapped BLAST and PSI-BLAST: a new generation of protein database programs. Nucleic $4 \quad$ Acids Res 25: 3389-3402.

5 Anaissie, E.J., Kuchar, R.T., Rex, J.H., Francesconi, A., Kasai, M., Muller, F.M., et al. (2011)

6 Fusariosis associated with pathogenic Fusarium species colonization of a hospital water 7 system: a new paradigm for the epidemiology of opportunistic mold pathogens. Clin Infect Dis $8 \quad 33: 1871-1878$.

9 Anaissie, E.J., Penzak, S.R., and Dignani, M.C. (2002) The hospital water supply as a source of 10 nosocomial infections: a plea for action. Arch Intern Med 162: 1483-1492.

11 Arie, T., Hayashi, Y., Nagatani, A., Furuya, M., and Yamaguchi, I. (1991) Production and partial 12 characterization of monoclonal antibodies against Fusarium oxysporum 860926a. Ann 13 Phytopathol Soc Jpn 57: 696-701.

14 Arie, T., Hayashi, Y., Yoneyama K, Nagatani, A., Furuya, M., and Yamaguchi, I. (1995) Detection 15 of Fusarium spp. in plants with monoclonal antibody. Ann Phytopathol Soc Jpn 61: 311-317.

16 Arrese, J.E., Piérard-Franchimont, C., and Piérard, G.E. (1996) Fatal hyalohyphomycosis following $17 \quad$ Fusarium onychomycosis in an immunocompromised patient. Am J Dermatopathol 18: 196$18 \quad 198$.

19 Bigley, V.H., Duarte, R.F., Gosling, R.D., Kibbler, C.C., Seaton, S., and Potter, M. (2004) 20 Fusarium dimerum infection in a stem cell transplant recipient treated successfully with 21 voriconazole. Bone Marrow Transplant 34: 815-817. 
Fusarium-specific monoclonal antibody

1 Bouchara, J-P., Hsieh, H.Y., Croquefer, S., Barton, R., Marchais, V., Pihet, M., et al. (2009)

2 Development of an oligonucleotide array for direct detection of fungi in sputum samples from 3 patients with cystic fibrosis. J Clin Microbiol 47: 142-152.

4 Boutati, E.I., and Anaissie, E.J. (1997) Fusarium, a significant emerging pathogen in patients with 5 haematological malignancies: ten years experience at a cancer centre and implications for $6 \quad$ management. Blood 90: 999-1008.

7 Chang, D.C., Grant, G.B., O’Donnell, K., Wannemuehler, K.A., Noble-Wang, J., Rao, C.Y., et al. 8 (2006) Multistate outbreak of Fusarium keratitis associated with use of a contact lens solution. 9 JAMA 296: 953-963.

10 Chow, T.Y.K., and Käfer, E. (1993) A rapid method for isolation of total nucleic acids from 11 Aspergillus nidulans. Fungal Genet Newsl 40: 25-27.

12 Danks, J.N., Rizvi, R.H., Barker, I., Turner, J.A., Rahman, S., and Northway, B.J. (1996) Specific 13 monoclonal antibodies to Fusarium species and Michrodochium nivale. Food Agric Immunol 8: $14 \quad 249-68$.

15 Davies, G., and Thornton, C.R. (2014) Differentiation of the emerging human pathogens 16 Trichosporon asahii and Trichosporon asteroides from other pathogenic yeasts and molds by $17 \quad$ using species-specific monoclonal antibodies. PLoS ONE 9: e84789.

18 Dignani, M.C., and Anaissie, E. (2004) Human fusariosis. Clin Microbiol Infect 10: 67-75.

19 Doggett, M.S. (2000) Characterisation of fungal biofilms within a municipal water distribution system. Appl Env Microbiol 66: 1249-1251.

21 Dursun, D., Fernandez, V., and Miller, D. (2003) Advanced Fusarium keratitis progressing to 22 endophthalmitis. Cornea 22: 300-303. 
Fusarium-specific monoclonal antibody

1 Edelstein, S.L., Akduman, L., Durham, B.H., Fothergill, A.W., and Hsu, H.Y. (2012) Resistant

$2 \quad$ Fusarium keratitis progressing to endophthalmitis. Ophthalmology Contact Lens 38: 331-335.

3 Feng, P., Qiaoyun, L., Najafzadeh, M.J., Gerrits van den Ende, A.H.G., Sun, J., Li, R., et al. (2014)

4 Cyphellophora and its relatives in Phialophora: biodiversity and possible role in human

5 disease. Fungal Diversity 65: 17-45.

6 Geiser, D.M., del Mar Jiménez-Gasco, M., Kang, S., Makalowska, I., Veeraraghavan, N., Ward,

7 T.D., et al. (2004) FUSARIUM-ID v.1.0: A DNA sequence database for identifying Fusarium.

$8 \quad$ Eur J Pl Pathol 110: 473-479.

9 Girmenia, C., Pagano, L., Corvatta, L., Mele, L., Del, A., Favero, P., et al. (2000) The

10 epidemiology of fusarioses in patients with haematological diseases. Br J Haematol 111: 27211276.

12 Gurusidappa, S.B., and Mamatha, H.S. (2011) Fusarial skin lesion in immunocompromised. Ind $J$ 13 Cancer 48: 116-117.

14 Hayashi, Y., Arie, T., Yoneyama, K., and Yamaguchi, I. (1998) Characterisation of the antigenic 15 determinant on Fusarium oxysporum recognized by a genus-specific monoclonal antibody. $J$ $16 \quad$ Gen Appl Microbiol 44: 43-47.

17 Hu, Z-Q., Liu, J-L., Li, H-P., Xing, S., Xue, S., Zhang, J-B., et al. (2012) Generation of a highly 18 reactive chicken-derived single-chain variable fragment against Fusarium verticillioides by 19 phage display. Int J Mol Sci 13: 7038-7056.

20 Hu, Z-Q., Li, H-P., Zhang, J-B., Huang, T., Liu, J-L., Xue, S., et al. (2013) A phage-displayed 21 chicken single-chain antibody fused to alkaline phosphatase detects Fusarium pathogens and 22 their presence in cereal grains. Anal Chim Acta 764: 84-92. 
Fusarium-specific monoclonal antibody

1 Jensen, T.G., Gahrn-Hansen, B., Arendrup, M., and Bruun, B. (2004) Fusarium fungaemia in 2 immunocompromised patients. Clin Microbiol Infect 10: 499-501.

3 Jensen, H.M.E., Aalbæk, B., Jungersen, G., Hartvig, T., Moser, C., Rozell, B.L., et al. (2011)

$4 \quad$ Immunohistochemical diagnosis of fusariosis with monoclonal antibodies. Mycoses 54: s545 s55.

6 Jurkunas, U., Behlau, I., and Colby, K. (2009) Fungal keratitis: changing pathogens and risk factors.

$7 \quad$ Cornea 28: 638-643.

8 Koehler, P., Tacke, D., and Cornely, O.A. (2014) Bone and joint infections by Mucorales, 9 Scedosporium, Fusarium and even rarer fungi. Crit Rev Microbiol. doi: $10 \quad 10.3109 / 1040841 X .2014 .910749$.

11 Lackner, M., Najafzadeh, M.J., Sun, J., Lu, Q., and de Hoog, G.S. (2012) Rapid identification of 12 Pseudallescheria and Scedosporium strains by using rolling circle amplification. Appl Environ $13 \quad$ Microbiol 78: 126-133.

14 Latenser, B.A. (2003) Fusarium infections in burn patients: a case report and review of the 15 literature. J Burn Care Rehabil 24: 285-288.

16 Macêdo, D.P.C., Neves, R.P., de Souza-Motta, C.M., and Magalhães, O.M.C. (2007) Engyodontium 17 album fungaemia: the first reported case. Braz J Microbiol 38: 110-112.

18 Mehl, H.L., and Epstein, L. (2008) Sewage and community shower drains are environmental 19 reservoirs of Fusarium species complex group 1, a human and plant pathogen. Env Microbiol 20 10: $219-227$.

21 Miceli, M.H., Díaz, J.A., and Lee, S.A. (2011) Emerging opportunistic yeast infections. Lancet 22 Infect Dis 11: 142-151. 
Fusarium-specific monoclonal antibody

1 Muhammed, M., Carneiro, H., Coleman, J., and Mylonakis, E. (2011) The challenge of managing 2 fusariosis. Virulence 2: 91-96.

3 Musa, H.O., Al Eisa, A., Halim, M., Sahovic, E., Gyger, M., Chaudhri, N., et al. (2000) The

4 spectrum of fusarium infection in immunocompromised patients with haematological 5 malignancies and in non-immunocompromised patients; A single institution experience over 10 $6 \quad$ years. Br J Haematol 108: 544-548.

7 Nucci, M., and Anaissie, E. (2002) Cutaneous infection by Fusarium species in healthy and 8 immunocompromised hosts: implications for diagnosis and management. Clin Infect Dis 35: $9909-920$.

10 Nucci, N., and Anaissie, E. (2007) Fusarium infections in immuncompromised patients. Clin 11 Microbiol Rev 20: 695-704.

12 O’Donnell, K., Sutton, D.A., Rinaldi, M.G., Sarver, B.A.J., Arunmozhi Balajee, S., Schroers, H-J., 13 et al. (2010) Internet-accessible DNA sequence database for identifying Fusaria from human $14 \quad$ and animal infections. J Clin Microbiol 48: 3708-3718.

15 Petrikkos, G., Skiada, A., Lortholary, O., Roilides, E., Walsh, T.J., and Kontoyiannis, D.P. (2012) 16 Epidemiology and clinical manifestations of mucormycosis. Clin Infect Dis 54: S23-S24.

17 Rougeron, A., Schuliar, G., Leto, J., Sitterle, E., Landry, D., Bougnoux, M.E., et al. (2014) Human18 impacted areas of France are environmental reservoirs of the Pseudallescheria 19 boydii/Scedosporium apiospermum species complex. Env Microbiol 17: 1039-1048.

20 Sandoval-Denis, M., Sutton, D.A., Cano-Lira, J.F., Gene, J., Fothergill, A.W., Wiederhold, N.P., et 21 al. (2014) Phylogeny of the clinically relevant species of the emerging fungus Trichoderma and 22 their antifungal susceptibilities. J Clin Microbiol 52: 2112-2125. 
Fusarium-specific monoclonal antibody

1 Schroers, H.-J., O’Donnell, K., Lamprecht, S.C., Kammophthalmologyr, P.L., Johnson, S., Sutton,

2 D.A., et al. (2009) Taxonomy and phylogeny of the Fusarium dimerum species group.

$3 \quad$ Mycologia 101: 44-70.

4 Short, D.P.G., O’Donnell, K., Zhang, N., Juba, J.H., and Geiser, D.M. (2011) Widespread

5 occurrence of diverse human pathogenic types of the fungus Fusarium detected in plumbing

$6 \quad$ drains. J Clin Microbiol 49: 4264-4272.

7 Steinmann, J., Schmidt, D., Buer, J., and Rath, P-M. (2011) Discrimination of Scedosporium

$8 \quad$ prolificans against Pseudallescheria boydii and Scedosporium apiospermum by semiautomated

9 repetitive sequence-based PCR. Med Mycol 49: 475-483.

10 Sutula, C.L., Gillett, J.M., Morrisey, S.M., and Ramsdell, D.C. (1986) Interpreting ELISA data and

11 establishing the positive-negative threshold. Plant Dis 70: 722-26.

12 Thamke, D.C., Mediratta, D.K., Dhabarde, A., and Shukla, A.K. (2015) Mycotic keratitis due to

13 Engyodontium album: first case report from India. Indian J Med Microbiol 33: 303-304.

14 Thornton, C.R. (2001) Immunological methods for fungi. In Molecular and Cellular Biology of

15 Filamentous Fungi, A Practical Approach. Talbot, N.J. (ed.). Oxford, UK: University Press, $16 \quad$ pp. $227-257$.

17 Thornton, C.R. (2008) Development of an immunochromatographic lateral flow device for rapid 18 serodiagnosis of invasive aspergillosis. Clin Vacc Immunol 15: 1095-1105.

19 Thornton, C.R. (2009) Tracking the emerging human pathogen Pseudallescheria boydii by using 20 highly specific monoclonal antibodies. Clin Vacc Immunol 16: 756-64.

21 Thornton, C.R. (2012) Serological techniques for diagnosis. In Fungal Plant Pathogens, Principles 22 and Protocols. Lane, C., Beales, P., Hughes, K. (eds.). Wallingford, UK: CABI, pp. 159-177. 
Fusarium-specific monoclonal antibody

1 Thornton, C.R., and Wills, O.E. (2015) Immunodetection of fungal and oomycete pathogens:

2 Established and emerging threats to human health, animal welfare, and global food security.

$3 \quad$ Crit Rev Microbiol. 41: 27-51.

4 Thornton, C.R., Ryder, L.S., Le Cocq, K., and Soanes, D.M. (2015) Identifying the emerging 5 human pathogen Scedosporium prolificans by using a species-specific monoclonal antibody 6 that binds to the melanin biosynthetic enzyme tetrahydroxynaphthalene reductase. Env $7 \quad$ Microbiol 17: 1023-1048.

8 Torres, H.J., and Kontoyiannis, D. (2011) Hyalohyphomycosis (hyaline molds). In Essentials of 9 Clinical Mycology, Kauffman, C.A., Pappas, P.G., Sobel, J.D., and Dismukes, W.E. (eds.). 10 New York, USA: Springer Science+Business Media, pp. 281-304.

11 Walsh, T.J., and Groll, A.H. (1999) Emerging fungal pathogens: evolving challenges to 12 immunocompromised patients for the twenty-first century. Transpl Infect Dis 1:247-261.

13 Wong, W.C., White, M., and Wright, I.G. (1988) Production of monoclonal antibodies to Fusarium $14 \quad$ oxysporum f.sp. cubense race 4. Lett Appl Microbiol 6: 39-42.

15 Zeng, J.S., Sutton, D.A., Fothergill, A.W., Rinaldi, M.G., Harrak, M.J., and de Hoog, G.S. (2007) 16 Spectrum of clinically relevant Exophiala species in the Unites States. J Clin Microbiol 45: $17 \quad 3713-3720$.

18 Zhang, N., O’Donnell, K., Sutton, D.A., Nalim, F.A., Summerbell, R.C., Padhye, A.A., et al. (2006) 19 Members of the Fusarium solani species complex that cause infections in both humans and 20 plants are common in the environment. J Clin Microbiol 44: 2186-2190. 
Fusarium-specific monoclonal antibody

1 Table 1. Locations and identities of sink swabs and water samples and results of ELISA tests and fungal

2 identification based on ITS sequencing.

\begin{tabular}{|c|c|c|c|c|c|c|}
\hline $\begin{array}{l}\text { Location } \\
\left(_{\text {Source })^{\text {a }}}\right.\end{array}$ & $\begin{array}{c}\text { Swab } \\
\text { ELISA abs } \\
(450 \mathrm{~nm})^{\mathrm{b}}\end{array}$ & $\begin{array}{c}\text { Mixed } \\
\text { culture } \\
\text { ELISA } \\
\text { abs } \\
(450 \mathrm{~nm})^{\mathrm{b}}\end{array}$ & $\begin{array}{l}\text { Isolate } \\
\text { No. }\end{array}$ & $\begin{array}{c}\text { Axenic } \\
\text { culture } \\
\text { ELISA } \\
\text { abs } \\
(450 \mathrm{~nm})^{\mathrm{b}}\end{array}$ & $\begin{array}{l}\text { Identification based on } \\
\text { ITS sequencing }\end{array}$ & $\begin{array}{c}\text { GenBank } \\
\text { Accession No. }\end{array}$ \\
\hline University (S1) & 0.0763 & 0.0575 & A1-1 & 1.3736 & Fusarium oxysporum & KT876668 \\
\hline University (S1) & \multirow{3}{*}{\multicolumn{2}{|c|}{$(2)$}} & A1-2 & 0.7646 & Fusarium oxysporum & KT876662 \\
\hline University (S1) & & & A1-3 & 0.0196 & Penicillium crustosum & KT876719 \\
\hline University (S1) & & & A1-4 & 0.0139 & Penicillium expansum & KT876718 \\
\hline University (S2) & 0.5472 & 0.0045 & A2-1 & 1.5723 & Fusarium solani & KT876635 \\
\hline University (S2) & & A $2-3$ & 0.6554 & Fusarium oxysporum & KT876690 \\
\hline University (S2) & & & A2-5 & 1.1312 & Fusarium solani & KT876631 \\
\hline University (S3) & 0.0053 & 0.0077 & A3-1 & 0.0082 & Trichosporon domesticum & KT876717 \\
\hline University (S3) & \multirow{3}{*}{\multicolumn{2}{|c|}{ 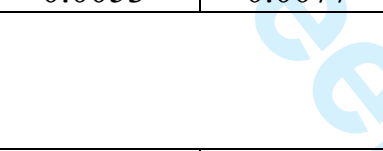 }} & A3-2 & 0.0162 & Cadophora fastigiata & KT876615 \\
\hline University (S3) & & & A3-3 & 0.0014 & Cyphellophora oxyspora & KT876613 \\
\hline University (S3) & & & A3-4 & 0.0093 & Penicillium crustosum & KT876714 \\
\hline University (S4) & \multirow{4}{*}{0.0297} & \multirow[t]{4}{*}{0.0049} & A4-1 & 0.0105 & Phoma herbarum & KT876697 \\
\hline University (S4) & & & A4-2 & 0.0079 & Penicillium echinulatum & KT876710 \\
\hline University (S4) & & & A4-3 & 0.0029 & Cytobasidium slooffiae & KT876704 \\
\hline University (S4) & & & A4-6 & 0.0060 & Trichoderma asperellum & KT876620 \\
\hline University (S5) & \multirow{6}{*}{\multicolumn{2}{|c|}{0.0039}} & A5-1 & 1.1815 & Fusarium oxysporum & KT876692 \\
\hline University (S5) & & & A5-2 & 0.0034 & Penicillium crustosum & KT876715 \\
\hline University (S5) & & & A5-5 & 0.0088 & Aspergillus niger & KT876702 \\
\hline University (S5) & & & A5-6 & 1.0630 & Fusarium oxysporum & KT876667 \\
\hline University (S5) & & & A5-7 & 0.0037 & $\begin{array}{l}\text { Rhodotorula } \\
\text { mucilaginosa }\end{array}$ & KT876700 \\
\hline University (S5) & & & A5-8 & 0.0088 & Cyphellophora oxyspora & KT876614 \\
\hline University (S6) & \multirow[t]{4}{*}{0.0251} & \multirow[t]{4}{*}{0.0412} & A6-1 & 1.1130 & Fusarium oxysporum & KT876648 \\
\hline University (S6) & & & A6-2 & 0.9410 & Fusarium oxysporum & KT876678 \\
\hline University (S6) & & & A6-3 & 0.6377 & Fusarium oxysporum & KT876688 \\
\hline University (S6) & & & A6-4 & 1.0020 & Fusarium solani & KT876640 \\
\hline University (S7) & \multirow{4}{*}{\multicolumn{2}{|c|}{0.0082}} & A7-1 & 0.0098 & Exophiala pisciphila & KT876618 \\
\hline University (S7) & & & A7-2 & 0.0096 & $\begin{array}{c}\text { Penicillium } \\
\text { brevicompactum }\end{array}$ & KT876695 \\
\hline University (S7) & & & A7-3 & 1.0556 & Fusarium oxysporum & KT876684 \\
\hline University (S7) & & & A7-4 & 1.0077 & Fusarium oxysporum & KT876671 \\
\hline University (S8) & \multirow[t]{3}{*}{0.0431} & \multirow[t]{3}{*}{0.0008} & $\mathrm{~A} 8-1$ & 1.1045 & Fusarium oxysporum & KT876672 \\
\hline University (S8) & & & A8-2 & 0.9707 & Fusarium oxysporum & KT876654 \\
\hline University (S8) & & & A8-3 & 0.0091 & Trichoderma atroviride & KT876622 \\
\hline University (S9) & \multirow[t]{4}{*}{0.3806} & \multirow[t]{4}{*}{0.0052} & A9-1 & 0.9087 & Fusarium solani & KT876639 \\
\hline University (S9) & & & A9-2 & 0.8849 & Fusarium solani & KT876638 \\
\hline University (S9) & & & A9-3 & 0.9004 & Fusarium solani & KT876632 \\
\hline University (S9) & & & A9-4 & 0.8093 & Fusarium solani & KT876636 \\
\hline
\end{tabular}


Fusarium-specific monoclonal antibody

\begin{tabular}{|c|c|c|c|c|c|c|}
\hline University (S10) & 0.0034 & 0.0028 & A10-1 & 0.0188 & Rhodotorula slooffiae & \\
\hline University (S10) & & & A10-2 & 0.0020 & Exophiala pisciphila & KT876616 \\
\hline University (S11) & 0.0231 & 0.0005 & B1-1 & 0.6064 & Fusarium dimerum & KT876625 \\
\hline University (S11) & & & B1-6 & 0.8138 & Fusarium dimerum & KT876628 \\
\hline University (S12) & 0.0200 & 0.0020 & B2-1 & 0.5678 & Fusarium dimerum & KT876626 \\
\hline University (S12) & & & B2-5 & 0.4827 & Fusarium dimerum & KT876624 \\
\hline University (S13) & 0.0091 & 0.0023 & B3-4 & 0.0026 & Phoma herbarum & KT876696 \\
\hline University (S14) & 0.0163 & 0.0008 & B4-1 & 0.6992 & Fusarium dimerum & KT876627 \\
\hline University (S15) & 0.0132 & 0.0003 & B5-1 & 0.8008 & Fusarium oxysporum & KT876674 \\
\hline University (S15) & & & B5-2 & 0.0048 & Mucor circinelloides & KT876701 \\
\hline University (S15) & & & B5-3 & 0.8851 & Fusarium oxysporum & KT876677 \\
\hline University (S16) & 0.0229 & 0.0002 & B6-1 & 0.8193 & Fusarium oxysporum & KT876676 \\
\hline University (S16) & & & B6-2 & 0.7582 & Fusarium oxysporum & KT876661 \\
\hline University (S17) & 0.0395 & 0.0014 & B7-1 & 0.8201 & Fusarium oxysporum & KT876675 \\
\hline University (S17) & & 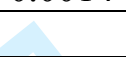 & B7-6 & 0.7758 & Fusarium dimerum & KT876623 \\
\hline University (S18) & 0.0133 & 0.0011 & B8-1 & 0.7347 & Fusarium solani & KT876637 \\
\hline University (S18) & & & B8-7 & 0.0095 & Rhodosporidium babjevae & KT876706 \\
\hline University (S19) & 0.0212 & 0.0048 & B9-1 & 0.0051 & $\begin{array}{c}\text { Meyerozyma } \\
\text { guilliermondii }\end{array}$ & KT876707 \\
\hline University (S19) & & & B9-2 & 0.8104 & Fusarium oxysporum & KT876657 \\
\hline University (S19) & & & B9-3 & 0.6302 & Fusarium solani & KT876634 \\
\hline University (S19) & & & B9-5 & 0.0017 & Penicillium crustosum & KT876720 \\
\hline University (S20) & 0.5467 & 0.0394 & $\mathrm{~B} 10-1$ & 0.7467 & Fusarium oxysporum & KT876680 \\
\hline University (S20) & & & $\mathrm{B} 10-2$ & 0.7667 & Fusarium oxysporum & KT876659 \\
\hline University (S20) & & & B10-6 & 0.0071 & Candida parapsilosis & KT876703 \\
\hline University (S20) & & & B10-7 & 0.0251 & $\begin{array}{l}\text { Meyerozyma } \\
\text { guilliermondii }\end{array}$ & KT876711 \\
\hline University (S20) & & & B10-9 & 0.0031 & Cystobasidium slooffiae & KT876712 \\
\hline University (S20) & & & B10-12 & 0.0251 & Trichosporon asteroides & KT876713 \\
\hline University (S21) & 0.0083 & 0.0269 & $\mathrm{C} 1-1$ & 0.0044 & Clavispora lusitaniae & KT876708 \\
\hline University (S21) & & & C1-3 & 0.8875 & Fusarium oxysporum & KT876682 \\
\hline University (S21) & & & $\mathrm{C} 1-4$ & 0.8884 & Fusarium dimerum & KT876629 \\
\hline University (S21) & & & $\mathrm{C} 1-7$ & 0.0003 & Exophiala pisciphila & KT876617 \\
\hline University (S22) & 0.2977 & 0.0047 & $\mathrm{C} 2-1$ & 0.9443 & Fusarium oxysporum & KT876658 \\
\hline University (S22) & & & $\mathrm{C} 2-4$ & 0.9825 & Fusarium oxysporum & KT876694 \\
\hline University (S22) & & & $\mathrm{C} 2-7$ & 0.8677 & Fusarium oxysporum & KT876687 \\
\hline University (S23) & 0.0808 & 0.0116 & $\mathrm{C} 3-1$ & 0.9853 & Fusarium oxysporum & KT876683 \\
\hline University (S23) & & & $\mathrm{C} 3-2$ & 1.0160 & Fusarium oxysporum & KT876693 \\
\hline University (S23) & & & $\mathrm{C} 3-4$ & 0.7485 & Fusarium oxysporum & KT876644 \\
\hline University (S24) & 0.1113 & 0.0202 & $\mathrm{C} 4-1$ & 0.8930 & Fusarium oxysporum & KT876670 \\
\hline University (S24) & & & $\mathrm{C} 4-2$ & 0.0028 & Candida intermedia & KT876709 \\
\hline University (S24) & & & $\mathrm{C} 4-5$ & 0.9008 & Fusarium dimerum & KT876630 \\
\hline University (S25) & 0.5741 & 0.0097 & C5-1 & 0.9874 & Fusarium oxysporum & KT876652 \\
\hline University (S25) & & & $\mathrm{C} 5-2$ & 1.0996 & Fusarium oxysporum & KT876656 \\
\hline University (S25) & & & $\mathrm{C} 5-3$ & 1.1236 & Fusarium oxysporum & KT876649 \\
\hline University (S25) & & & $\mathrm{C} 5-4$ & 1.0355 & Fusarium oxysporum & KT876669 \\
\hline University (S25) & & & $\mathrm{C} 5-5$ & 1.0672 & Fusarium oxysporum & KT876660 \\
\hline University (S26) & 0.0603 & 0.0348 & C6-1 & 1.0135 & Fusarium oxysporum & KT876665 \\
\hline
\end{tabular}


Fusarium-specific monoclonal antibody

\begin{tabular}{|c|c|c|c|c|c|c|}
\hline University (S26) & & & C6-2 & 0.9362 & Fusarium oxysporum & KT876681 \\
\hline University (S26) & & & C6-3 & 0.8898 & Fusarium oxysporum & \\
\hline University (S26) & & & C6-4 & 1.0577 & Fusarium oxysporum & KT876686 \\
\hline University (S26) & & & C6-5 & 0.9445 & Fusarium oxysporum & KT876646 \\
\hline University (S27) & 0.1279 & 0.0113 & $\mathrm{C} 7-1$ & 0.9724 & Fusarium oxysporum & KT876666 \\
\hline University (S28) & 0.0607 & 0.0035 & $\mathrm{C} 8-1$ & 0.8694 & Fusarium oxysporum & KT876685 \\
\hline University (S28) & & & $\mathrm{C} 8-2$ & 0.9599 & Fusarium oxysporum & KT876655 \\
\hline University (S28) & & & $\mathrm{C} 8-3$ & 0.0031 & Penicillium expansum & KT876716 \\
\hline University (S28) & & & $\mathrm{C} 8-4$ & 0.0049 & Phoma herbarum & KT876698 \\
\hline University (S28) & & & C8-6 & 0.9003 & Fusarium oxysporum & KT876679 \\
\hline University (S29) & 0.0310 & 0.0001 & C9-1 & 1.0488 & Fusarium oxysporum & KT876651 \\
\hline University (S29) & & & C9-2 & 0.9254 & Fusarium oxysporum & KT876645 \\
\hline University (S29) & & & C9-3 & 0.9711 & Fusarium oxysporum & KT876664 \\
\hline University (S29) & & & C9-4 & 1.035 & Fusarium oxysporum & KT876647 \\
\hline University (S29) & & & C9-5 & 0.9604 & Fusarium oxysporum & KT876663 \\
\hline University (S30) & 0.1002 & 0.0333 & C10-1 & 0.9254 & Fusarium oxysporum & KT876689 \\
\hline University (S30) & & & $\mathrm{C} 10-2$ & 1.0198 & Fusarium solani & KT876642 \\
\hline University (S30) & & & C10-4 & 0.9007 & Fusarium solani & KT876641 \\
\hline University (S30) & & & $\mathrm{C} 10-7$ & 1.0697 & Fusarium solani & KT876643 \\
\hline University (S31) & 0.6175 & 0.1129 & CRT1-1 & 0.4639 & Fusarium oxysporum & KT876691 \\
\hline University (S31) & & & CRT1-2 & 0.9086 & Fusarium oxysporum & KT876650 \\
\hline University (S31) & & & CRT1-3 & 0.7503 & Fusarium oxysporum & KT876652 \\
\hline University (S32) & 0.0303 & 0.0021 & CRT2-1 & 0.0040 & Trichoderma asperellum & KT876619 \\
\hline University (S32) & & & CRT2-2 & 0.0417 & Trichoderma asperellum & KT876621 \\
\hline University (S33) & 0.0663 & 0.0028 & CRT3-1 & 0.0256 & Phoma herbarum & KT876699 \\
\hline ICU (S34) & 0.0285 & 0.3620 & R1-1 & 1.5057 & Fusarium solani & KT876550 \\
\hline ICU (S34) & & & R1-2 & 1.5154 & Fusarium solani & KT876551 \\
\hline ICU (S34) & & & R1-3 & 1.5555 & Fusarium solani & KT876549 \\
\hline ICU (S35) & 0.0071 & 0.0147 & R2-4 & 0.0096 & Clonostachys rosea & KT876552 \\
\hline ICU (S35) & & & R2-5 & 0.0018 & Clonostachys rosea & KT876553 \\
\hline ICU (S35) & & & $\mathrm{R} 2-6$ & 0.0035 & Clonostachys rosea & KT876554 \\
\hline ICU (S36) & 0.0391 & 0.045 & R3-1 & 0.0006 & Trichoderma asperellum & KT876548 \\
\hline ITU (S37) & 0.7291 & 0.7724 & R5-1 & 1.5606 & Fusarium dimerum & KT876561 \\
\hline ITU (S37) & & & R5-2 & 1.3481 & Fusarium dimerum & KT876565 \\
\hline ITU (S37) & & & R5-3 & 1.4990 & Fusarium dimerum & KT876567 \\
\hline ITU (S38) & 1.2086 & 0.3691 & R6-1 & 1.3388 & Fusarium dimerum & KT876572 \\
\hline ITU (S38) & & & R6-2 & 1.4734 & Fusarium dimerum & KT876563 \\
\hline ITU (S38) & & & R6-3 & 1.4312 & Fusarium dimerum & KT876562 \\
\hline ITU (S38) & & & R6-9 & 1.2648 & Fusarium oxysporum & KT876557 \\
\hline ITU (S39) & 0.1121 & 0.8186 & R7-1 & 1.3266 & Fusarium dimerum & KT876570 \\
\hline ITU (S39) & & & R7-2 & 1.2352 & Fusarium dimerum & KT876564 \\
\hline ITU (S40) & 1.0157 & 0.3612 & R8-1 & 1.2399 & Fusarium dimerum & KT876568 \\
\hline ITU (S40) & & & R8-2 & 1.1552 & Fusarium dimerum & KT876566 \\
\hline ITU (S40) & & & $\mathrm{R} 8-3$ & 1.1856 & Fusarium dimerum & KT876558 \\
\hline ITU (S41) & 0.0391 & 0.0477 & R9-3 & 0.0113 & $\begin{array}{c}\text { Exophiala } \\
\text { phaeomuriformis }\end{array}$ & KT876555 \\
\hline ITU (S42) & 0.0549 & 0.0097 & R10-2 & 0.0072 & Cadophora fastigiata & KT876556 \\
\hline ITU (S42) & & & R10-6 & 0.0124 & Coniochaeta fasciculata & KT876721 \\
\hline ITU (S43) & 0.0630 & 1.5751 & $\mathrm{X} 2-2$ & 1.1492 & Fusarium dimerum & KT876571 \\
\hline
\end{tabular}


Fusarium-specific monoclonal antibody

\begin{tabular}{|c|c|c|c|c|c|c|}
\hline ITU (S43) & & & $\mathrm{X} 2-4$ & 1.2505 & Fusarium dimerum & KT876560 \\
\hline ITU (S43) & & & $\mathrm{X} 2-5$ & 1.1397 & Fusarium dimerum & KT876569 \\
\hline ITU (S43) & & & $\mathrm{X} 2-6$ & 1.1716 & Fusarium dimerum & KT876559 \\
\hline $\begin{array}{l}\text { Ophthalmology } \\
\text { Unit (S44) }\end{array}$ & 1.0313 & 1.5035 & X3-1 & 1.1353 & Fusarium dimerum & KT876509 \\
\hline $\begin{array}{l}\text { Ophthalmology } \\
\text { Unit (S44) }\end{array}$ & & & X3-2 & 0.0224 & Candida parapsilosis & KT876498 \\
\hline $\begin{array}{l}\text { Ophthalmology } \\
\text { Unit (S44) }\end{array}$ & & & X3-3 & 1.1856 & Fusarium dimerum & KT876512 \\
\hline $\begin{array}{l}\text { Ophthalmology } \\
\text { Unit (S45) }\end{array}$ & 1.4582 & 1.4049 & $\mathrm{X} 4-1$ & 1.1322 & Fusarium dimerum & KT876511 \\
\hline $\begin{array}{l}\text { Ophthalmology } \\
\text { Unit (S45) }\end{array}$ & & & $\mathrm{X} 4-2$ & 1.1523 & Fusarium dimerum & KT876500 \\
\hline $\begin{array}{l}\text { Ophthalmology } \\
\text { Unit (S45) }\end{array}$ & & & $X 4-3$ & 0.0622 & Candida parapsilosis & KT876508 \\
\hline $\begin{array}{l}\text { Ophthalmology } \\
\text { Unit (S45) }\end{array}$ & & & $\mathrm{X} 4-4$ & 0.1245 & Gloeotinia temulenta & KT876515 \\
\hline $\begin{array}{l}\text { Ophthalmology } \\
\text { Unit (S46) }\end{array}$ & 0.3325 & 1.4466 & $\mathrm{X} 5-2$ & 1.0654 & Fusarium dimerum & KT876510 \\
\hline $\begin{array}{l}\text { Ophthalmology } \\
\text { Unit (S46) }\end{array}$ & & & $\mathrm{X} 5-3$ & 0.0599 & $\begin{array}{l}\text { Rhodotorula } \\
\text { mucilaginosa }\end{array}$ & KT876501 \\
\hline $\begin{array}{l}\text { Ophthalmology } \\
\text { Unit (S46) }\end{array}$ & & & $\times 5-4$ & 1.0862 & Fusarium dimerum & KT876513 \\
\hline $\begin{array}{l}\text { Ophthalmology } \\
\text { Unit (S47) }\end{array}$ & 0.2640 & 1.3729 & X6-1 & 0.0616 & Candida parapsilosis & KT876499 \\
\hline $\begin{array}{l}\text { Ophthalmology } \\
\text { Unit (S47) }\end{array}$ & & & $\mathrm{X} 6-2$ & 0.063 & Engyodontium album & KT876522 \\
\hline $\begin{array}{l}\text { Ophthalmology } \\
\text { Unit (S47) }\end{array}$ & & & X6-3 & 0.0603 & Engyodontium album & KT876521 \\
\hline $\begin{array}{l}\text { Ophthalmology } \\
\text { Unit (S47) }\end{array}$ & & & X6-4 & 0.0657 & $\begin{array}{c}\text { Exophiala } \\
\text { phaeomuriformis }\end{array}$ & KT876504 \\
\hline $\begin{array}{l}\text { Ophthalmology } \\
\text { Unit (S48) }\end{array}$ & 0.5299 & 0.0223 & $\mathrm{X} 8-2$ & 0.0118 & $\begin{array}{l}\text { Cladosporium } \\
\text { macrocarpum }\end{array}$ & KT876506 \\
\hline $\begin{array}{l}\text { Ophthalmology } \\
\text { Unit (S48) }\end{array}$ & & & $\mathrm{X} 8-3$ & 0.0985 & Gloeotinia temulenta & KT876514 \\
\hline $\begin{array}{l}\text { Ophthalmology } \\
\text { Unit (S48) }\end{array}$ & & & $X 8-4$ & 0.0662 & Engyodontium album & KT876523 \\
\hline $\begin{array}{l}\text { Ophthalmology } \\
\text { Unit (S49) }\end{array}$ & 0.1872 & 0.0061 & X9-1 & 0.0686 & Engyodontium album & KT876520 \\
\hline $\begin{array}{l}\text { Ophthalmology } \\
\text { Unit (S49) }\end{array}$ & & & X9-2 & 0.0611 & Candida parapsilosis & KT876496 \\
\hline $\begin{array}{l}\text { Ophthalmology } \\
\text { Unit (S49) }\end{array}$ & & & X9-3 & 0.0641 & Candida parapsilosis & KT876497 \\
\hline $\begin{array}{l}\text { Ophthalmology } \\
\text { Unit (S49) }\end{array}$ & & & X9-4 & 0.0677 & Exophiala dermatitidis & KT876503 \\
\hline $\begin{array}{l}\text { Ophthalmology } \\
\text { Unit (S49) }\end{array}$ & & & $X 9-5$ & 0.0705 & Engyodontium album & KT876519 \\
\hline $\begin{array}{l}\text { Ophthalmology } \\
\text { Unit (S49) }\end{array}$ & & & X9-7 & 0.0660 & Exophiala pisciphila & KT876502 \\
\hline
\end{tabular}


Fusarium-specific monoclonal antibody

\begin{tabular}{|c|c|c|c|c|c|c|}
\hline $\begin{array}{l}\text { Ophthalmology } \\
\text { Unit (T1) }\end{array}$ & 0.0132 & 0.0159 & $\mathrm{X} 10-1$ & 0.0868 & $\begin{array}{c}\text { Cladosporium } \\
\text { sphaerospermum }\end{array}$ & KT876507 \\
\hline $\begin{array}{l}\text { Ophthalmology } \\
\text { Unit (T1) }\end{array}$ & & & $\mathrm{X} 10-3$ & 0.0130 & Engyodontium album & KT876518 \\
\hline $\begin{array}{l}\text { Ophthalmology } \\
\text { Unit (T2) }\end{array}$ & 0.0117 & 0.0883 & W1-1 & 0.0114 & Paraconiothyrium fuckelii & KT876505 \\
\hline $\begin{array}{c}\text { Ophthalmology } \\
\text { Unit (T2) }\end{array}$ & & & W1-2 & 0.0139 & Engyodontium album & KT876517 \\
\hline $\begin{array}{l}\text { Ophthalmology } \\
\text { Unit (T2) }\end{array}$ & & & W1-3 & 0.0108 & Engyodontium album & KT876516 \\
\hline $\begin{array}{c}\text { Haematology } \\
\text { Unit (S50) }\end{array}$ & 0.2694 & 0.0093 & $\mathrm{~K} 1-1$ & 0.0172 & Trichoderma asperellum & KT876534 \\
\hline $\begin{array}{l}\text { Haematology } \\
\text { Unit (S51) }\end{array}$ & 0.7466 & 1.1746 & K2-1 & 0.0144 & Trichoderma viride & KT876533 \\
\hline $\begin{array}{l}\text { Haematology } \\
\text { Unit (S51) }\end{array}$ & & & $\mathrm{K} 2-3$ & 1.1385 & Fusarium solani & KT876543 \\
\hline $\begin{array}{l}\text { Haematology } \\
\text { Unit (S51) }\end{array}$ & & & $\mathrm{K} 2-4$ & 1.2487 & Fusarium solani & KT876542 \\
\hline $\begin{array}{c}\text { Haematology } \\
\text { Unit (S52) }\end{array}$ & 1.0621 & 1.3090 & K6-1 & 1.1544 & Fusarium oxysporum & KT876541 \\
\hline $\begin{array}{l}\text { Haematology } \\
\text { Unit (S52) }\end{array}$ & & & K6-4 & 0.0071 & Candida parapsilosis & KT876524 \\
\hline $\begin{array}{l}\text { Haematology } \\
\text { Unit (S53) }\end{array}$ & 1.0120 & 1.2628 & K8-1 & 1.2376 & Fusarium solani & KT876722 \\
\hline $\begin{array}{l}\text { Haematology } \\
\text { Unit (S53) }\end{array}$ & & & $\mathrm{K} 8-2$ & 1.2983 & Fusarium solani & KT876723 \\
\hline $\begin{array}{l}\text { Haematology } \\
\text { Unit (S53) }\end{array}$ & & & K8-6 & 0.0084 & Cadophora fastigiata & KT876531 \\
\hline $\begin{array}{l}\text { Haematology } \\
\text { Unit (S53) }\end{array}$ & & & K8-7 & 0.0051 & Trichoderma asperellum & KT876535 \\
\hline $\begin{array}{l}\text { Haematology } \\
\text { Unit (S54) }\end{array}$ & 0.9843 & 1.2903 & K9-1 & 0.0046 & Candida parapsilosis & KT876525 \\
\hline $\begin{array}{c}\text { Haematology } \\
\text { Unit (S54) }\end{array}$ & & & K9-2 & 0.0090 & Rhodotorula dairenensis & KT876526 \\
\hline $\begin{array}{l}\text { Haematology } \\
\text { Unit (S54) }\end{array}$ & & & K9-4 & 0.0073 & Cadophora fastigiata & KT876532 \\
\hline $\begin{array}{l}\text { Haematology } \\
\text { Unit (S54) }\end{array}$ & & & K9-5 & 1.1652 & Fusarium solani & KT876547 \\
\hline $\begin{array}{l}\text { Haematology } \\
\text { Unit (S54) }\end{array}$ & & & K9-6 & 1.0316 & Fusarium solani & KT876545 \\
\hline $\begin{array}{l}\text { Haematology } \\
\text { Unit (S55) }\end{array}$ & 0.8638 & 1.0753 & K10-1 & 1.0684 & Fusarium solani & KT876546 \\
\hline $\begin{array}{l}\text { Haematology } \\
\text { Unit (S55) }\end{array}$ & & & $\mathrm{K} 10-2$ & 1.0386 & Fusarium solani & KT876544 \\
\hline $\begin{array}{l}\text { Haematology } \\
\text { Unit (W50) }\end{array}$ & 0.0082 & 0.0451 & G1-2 & 0.0097 & Exophiala pisciphila & KT876529 \\
\hline $\begin{array}{l}\text { Haematology } \\
\text { Unit (W51) }\end{array}$ & 0.0581 & 0.0553 & $\mathrm{G} 2-1$ & 0.0104 & Engyodontium album & KT876540 \\
\hline $\begin{array}{l}\text { Haematology } \\
\text { Unit (W51) }\end{array}$ & & & G2-3 & 0.0062 & Exophiala pisciphila & KT876530 \\
\hline
\end{tabular}


Fusarium-specific monoclonal antibody

\begin{tabular}{|c|c|c|c|c|c|c|}
\hline $\begin{array}{l}\text { Haematology } \\
\text { Unit (W52) }\end{array}$ & 0.0125 & 0.0118 & G6-2 & 0.0161 & Exophiala castellanii & KT876528 \\
\hline $\begin{array}{l}\text { Haematology } \\
\text { Unit (W53) }\end{array}$ & 0.0075 & 0.0219 & G8-1 & 0.0065 & Engyodontium album & KT876538 \\
\hline $\begin{array}{c}\text { Haematology } \\
\text { Unit (W54) }\end{array}$ & 0.0002 & 0.0164 & G9-2 & 0.0005 & Gloeotinia temulenta & KT876537 \\
\hline $\begin{array}{l}\text { Haematology } \\
\text { Unit (W55) }\end{array}$ & 0.0021 & 0.0182 & G10-1 & 0.0051 & Engyodontium album & KT876539 \\
\hline $\begin{array}{l}\text { Oncology Unit } \\
\text { (S56) }\end{array}$ & 0.4822 & 1.2287 & H1-1 & 0.9022 & Fusarium dimerum & KT876595 \\
\hline $\begin{array}{l}\text { Oncology Unit } \\
\text { (S56) }\end{array}$ & $\sqrt{V}$ & & $\mathrm{H} 1-3$ & 0.0151 & Pichia kudriavzevii & KT876578 \\
\hline $\begin{array}{c}\text { Oncology Unit } \\
\text { (S57) }\end{array}$ & 1.0579 & 1.1650 & $\mathrm{H} 2-1$ & 0.9312 & Fusarium dimerum & KT876590 \\
\hline $\begin{array}{c}\text { Oncology Unit } \\
\text { (S57) }\end{array}$ & & & $\mathrm{H} 2-5$ & 0.0061 & Magnusiomyces capitatus & KT876611 \\
\hline $\begin{array}{c}\text { Oncology Unit } \\
\text { (S58) }\end{array}$ & 1.0938 & 1.2091 & H3-1 & 0.8909 & Fusarium oxysporum & KT876584 \\
\hline $\begin{array}{c}\text { Oncology Unit } \\
\text { (S58) }\end{array}$ & & & $\mathrm{H} 3-4$ & 0.0075 & Candida palmioleophila & KT876573 \\
\hline $\begin{array}{c}\text { Oncology Unit } \\
\text { (S58) }\end{array}$ & & & $\mathrm{H} 3-5$ & 0.0140 & Rhodotorula glutinis & KT876598 \\
\hline $\begin{array}{c}\text { Oncology Unit } \\
\text { (S59) }\end{array}$ & 0.0253 & 1.0856 & H4-1 & 0.8884 & Fusarium dimerum & KT876596 \\
\hline $\begin{array}{c}\text { Oncology Unit } \\
\text { (S59) }\end{array}$ & & & $\mathrm{H} 4-3$ & 0.0004 & Magnusiomyces capitatus & KT876612 \\
\hline $\begin{array}{c}\text { Oncology Unit } \\
(\mathrm{S} 60)\end{array}$ & 1.0856 & 1.0193 & $\mathrm{H} 5-1$ & 1.1148 & Fusarium dimerum & KT876593 \\
\hline $\begin{array}{l}\text { Oncology Unit } \\
(\text { S60) }\end{array}$ & & & H5-3 & 0.005 & Candida tropicalis & KT876574 \\
\hline $\begin{array}{c}\text { Oncology Unit } \\
\text { (S61) }\end{array}$ & 0.5256 & 1.1898 & H6-1 & 1.1238 & Fusarium dimerum & KT876594 \\
\hline $\begin{array}{l}\text { Oncology Unit } \\
\text { (S62) }\end{array}$ & 1.0789 & 1.1588 & $\mathrm{H} 7-1$ & 1.1513 & Fusarium oxysporum & KT876583 \\
\hline $\begin{array}{l}\text { Oncology Unit } \\
\text { (S62) }\end{array}$ & & & $\mathrm{H} 7-3$ & 0.0045 & Candida tropicalis & KT876575 \\
\hline $\begin{array}{c}\text { Oncology Unit } \\
\text { (S63) }\end{array}$ & 0.0953 & 0.0152 & H8-1 & 0.0063 & Phoma herbarum & KT876580 \\
\hline $\begin{array}{c}\text { Oncology Unit } \\
\text { (S63) }\end{array}$ & & & $\mathrm{H} 8-3$ & 0.0126 & Candida albicans & KT876577 \\
\hline $\begin{array}{c}\text { Oncology Unit } \\
\text { (S63) }\end{array}$ & & & $\mathrm{H} 8-4$ & 0.0088 & $\begin{array}{l}\text { Rhodotorula } \\
\text { mucilaginosa }\end{array}$ & KT876599 \\
\hline $\begin{array}{c}\text { Oncology Unit } \\
\text { (S64) }\end{array}$ & 0.1926 & 0.0162 & H9-1 & 0.0152 & Exophiala dermatitidis & KT876581 \\
\hline $\begin{array}{c}\text { Oncology Unit } \\
\text { (S64) }\end{array}$ & & & H9-2 & 0.0049 & Candida orthopsilosis & KT876576 \\
\hline $\begin{array}{c}\text { Oncology Unit } \\
\text { (S64) }\end{array}$ & & & H9-3 & 0.0109 & Pichia kudriavzevii & KT876579 \\
\hline $\begin{array}{l}\text { Oncology Unit } \\
\text { (S65) }\end{array}$ & 0.4961 & 1.1005 & H10-1 & 0.9606 & Fusarium dimerum & KT876589 \\
\hline
\end{tabular}


Fusarium-specific monoclonal antibody

\begin{tabular}{|c|c|c|c|c|c|c|}
\hline $\begin{array}{l}\text { Oncology Unit } \\
(\text { S65) }\end{array}$ & & & $\mathrm{H} 10-4$ & 0.0163 & Trametes ochracea & KT876608 \\
\hline $\begin{array}{l}\text { Oncology Unit } \\
\text { (W56) }\end{array}$ & 0.0030 & 0.0247 & P1-2 & 0.0082 & Exophiala dermatitidis & KT876582 \\
\hline $\begin{array}{l}\text { Oncology Unit } \\
\text { (W56) }\end{array}$ & & & P1-3 & 0.0024 & Trametes versicolor & KT876603 \\
\hline $\begin{array}{l}\text { Oncology Unit } \\
\text { (W57) }\end{array}$ & 0.0061 & 1.0891 & P2-1 & 0.8451 & Fusarium dimerum & KT876587 \\
\hline $\begin{array}{l}\text { Oncology Unit } \\
\text { (W57) }\end{array}$ & & & P2-2 & 0.9429 & Fusarium dimerum & KT876597 \\
\hline $\begin{array}{l}\text { Oncology Unit } \\
\text { (W57) }\end{array}$ & & & $\mathrm{P} 2-3$ & 0.8627 & Fusarium dimerum & KT876588 \\
\hline $\begin{array}{l}\text { Oncology Unit } \\
\text { (W58) }\end{array}$ & 0.0016 & 0.0202 & P3-1 & 0.0177 & Trametes ochracea & KT876607 \\
\hline $\begin{array}{l}\text { Oncology Unit } \\
\text { (W59) }\end{array}$ & 0.0035 & 0.0125 & P4-1 & 0.0164 & Beauveria bassiana & KT876586 \\
\hline $\begin{array}{l}\text { Oncology Unit } \\
\text { (W59) }\end{array}$ & & & P4-2 & 0.0084 & Gliomastix polychroma & KT876584 \\
\hline $\begin{array}{l}\text { Oncology Unit } \\
\text { (W60) }\end{array}$ & 0.0025 & 1.1746 & P5-1 & 0.8563 & Fusarium dimerum & KT876591 \\
\hline $\begin{array}{l}\text { Oncology Unit } \\
\text { (W60) }\end{array}$ & & & P5-2 & 0.8066 & Fusarium dimerum & KT876592 \\
\hline $\begin{array}{l}\text { Oncology Unit } \\
\text { (W60) }\end{array}$ & & & P5-3 & 0.0024 & Piptoporus betulinus & KT876609 \\
\hline $\begin{array}{l}\text { Oncology Unit } \\
\text { (W62) }\end{array}$ & 0.0011 & 0.0417 & P7-1 & 0.0097 & Trametes versicolor & KT876605 \\
\hline $\begin{array}{l}\text { Oncology Unit } \\
\text { (W63) }\end{array}$ & 0.0044 & 0.0060 & P8-1 & 0.0144 & Trametes versicolor & KT876606 \\
\hline $\begin{array}{l}\text { Oncology Unit } \\
\text { (W63) }\end{array}$ & & & P8-2 & 0.0106 & Stereum gausapatum & KT876601 \\
\hline $\begin{array}{l}\text { Oncology Unit } \\
\text { (W64) }\end{array}$ & 0.0022 & 0.0163 & P9-1 & 0.0101 & Stereum gausapatum & KT876600 \\
\hline $\begin{array}{l}\text { Oncology Unit } \\
\text { (W64) }\end{array}$ & & & P9-2 & 0.0068 & Trametes versicolor & KT876604 \\
\hline $\begin{array}{l}\text { Oncology Unit } \\
\text { (W64) }\end{array}$ & & & P9-3 & 0.0140 & Stereum gausapatum & KT876602 \\
\hline $\begin{array}{l}\text { Oncology Unit } \\
\text { (W65) }\end{array}$ & 0.0051 & 0.0280 & P10-1 & 0.0032 & $\begin{array}{c}\text { Phaeophlebiopsis } \\
\text { peniophoroides }\end{array}$ & KT876610 \\
\hline
\end{tabular}

1 a. S, sink; W, tap water from corresponding sink number; $\mathrm{T}$, water sample from main tank.

2 b. Threshold absorbance value for detection in ELISA $\geq 0.100$. Shading indicates earliest point in 3 sampling process at which antigen was detectable in ELISA tests with ED7.

4 c. Fusarium strains in bold further characterized by TEF-1 $\alpha$ PCR analysis (Table S3 and Supporting 5 Data Set 1). 
Fusarium-specific monoclonal antibody

\section{Figure legends}

Figure 1. Specificity of ED7 determined by Enzyme-Linked Immunosorbent Assay tests of surface washings containing water-soluble antigens from Fusarium species and related and unrelated yeasts and molds. (A) ELISA absorbance values at $450 \mathrm{~nm}$ for antigens from $F$. solani and unrelated yeasts and molds and (B) for antigens from the F. solani teleomorph Haematonectria haematococca and related Fusarium species. Wells were coated with $60 \mu \mathrm{g}$ protein $\mathrm{ml}^{-1}$ buffer. Bars are the means of three biological replicates \pm standard errors and the threshold absorbance value for detection of antigen in ELISA is $\geq 0.100$ (indicated by lines on graphs). Numbers in parentheses after species names denote strain numbers with further details of strains provided in Table S1.

Figure 2. Characterisation of the ED7 antigen and its epitope and spatial distribution of the antigen in spores and hyphae. (A) Western immunoblot with ED7 using culture fluid from 2-d-old PDB cultures of $F$. solani CBS224.34 (lane 1) and F. oxysporum f.sp. lycopersici CBS167.30 (lane 2). Wells were loaded with $1.6 \mu \mathrm{g}$ of protein. $\mathrm{M}_{\mathrm{r}}$ denotes molecular weight in $\mathrm{kDa}$. Note the major extracellular antigen with molecular weight of $\sim 200 \mathrm{kDa}$. (B) Absorbance values from ELISA tests with ED7 using surface washings containing water-soluble antigens immobilized to the wells of microtitre plates and treated with trypsin or PBS only (control) at $4^{\circ} \mathrm{C}$ and $37^{\circ} \mathrm{C}$. Bars are the means of three biological replicates \pm standard errors and bars with the same letter are not significantly different at $\mathrm{p}<0.001$ (ANOVA and Tukey-Kramer test). (C) Absorbance values from ELISA tests with ED7 using surface washings containing water-soluble antigens immobilized to the wells of microtitre plates and treated with pronase or Milli-Q $\mathrm{H}_{2} \mathrm{O}$ only (control) at $4^{\circ} \mathrm{C}$ and $37^{\circ} \mathrm{C}$. Bars are the means of three biological replicates \pm standard errors and bars with the same letter are not significantly different at $\mathrm{p}<0.001$ (ANOVA and Tukey-Kramer test). (D) Stability of the water- 
Fusarium-specific monoclonal antibody

1 soluble ED7 antigen following heating of surface washings at $100^{\circ} \mathrm{C}$ over a 70 min period. Treated

2 antigen was subsequently immobilized to the wells of microtitre plates and assayed by ELISA. Bars

3 are the means of three biological replicates \pm standard errors and bars with the same letter are not

4 significantly different at $\mathrm{p}<0.001$ (ANOVA and Tukey-Kramer test). (E) Absorbance values from

5 ELISA tests with ED7 using surface washings containing water-soluble antigens immobilized to the

6 wells of microtitre plates and treated with periodate (open circles) or with acetate only control

7 (closed circles) at $4^{\circ} \mathrm{C}$ over a $20 \mathrm{~h}$ period. Each point is the mean of three biological replicates \pm

8 standard errors. (F to I) Photomicrographs of F. solani CBS224.34 immunostained with ED7 or

9 TCM control and goat anti-mouse polyvalent Ig fluorescein isothiocyanate (FITC) conjugate. (F)

10 Brightfield image of germinated conidium with hypha probed with ED7 followed by fluorochrome

11 conjugate (scale bar $=6 \mu \mathrm{m})$; $(\mathrm{G})$ Same field of view as panel $\mathrm{F}$ but examined under

12 epifluorescence. Note intense staining of the cell wall of microconidium and hypha; (H) Brightfield

13 image of germinated conidium with hypha probed with TCM (negative control) followed by FITC

14 conjugate (scale bar $=3 \mu \mathrm{m}$ ); (I) Same field of view as panel $\mathrm{H}$ but examined under

15 epifluorescence. Note lack of staining, demonstrating specific binding of ED7 to surface antigen. (J-

16 L) Immunogold labeling of sections of conidia and hyphae of $F$. solani CBS224.34. (J) Transverse

17 section of conidium incubated with ED7 and anti-mouse immunoglobulin $20 \mathrm{~nm}$ gold particles,

18 showing antigen in the cell wall and in an extracellular fimbrial matrix surrounding the spore (scale

19 bar $=250 \mathrm{~nm}) ;(\mathrm{K})$ Longitudinal section of hypha incubated with ED7 and anti-mouse

20 immunoglobulin $20 \mathrm{~nm}$ gold particles, showing antigen in the cell wall and in an extracellular

21 matrix surrounding the cell (scale bar $=100 \mathrm{~nm}$ ); (L) Transverse section of a conidium incubated

22 with TCM (negative control) and anti-mouse immunoglobulin $20 \mathrm{~nm}$ gold particles, showing lack

23 of staining by the secondary gold conjugate (scale bar $=180 \mathrm{~nm})$. 
A.

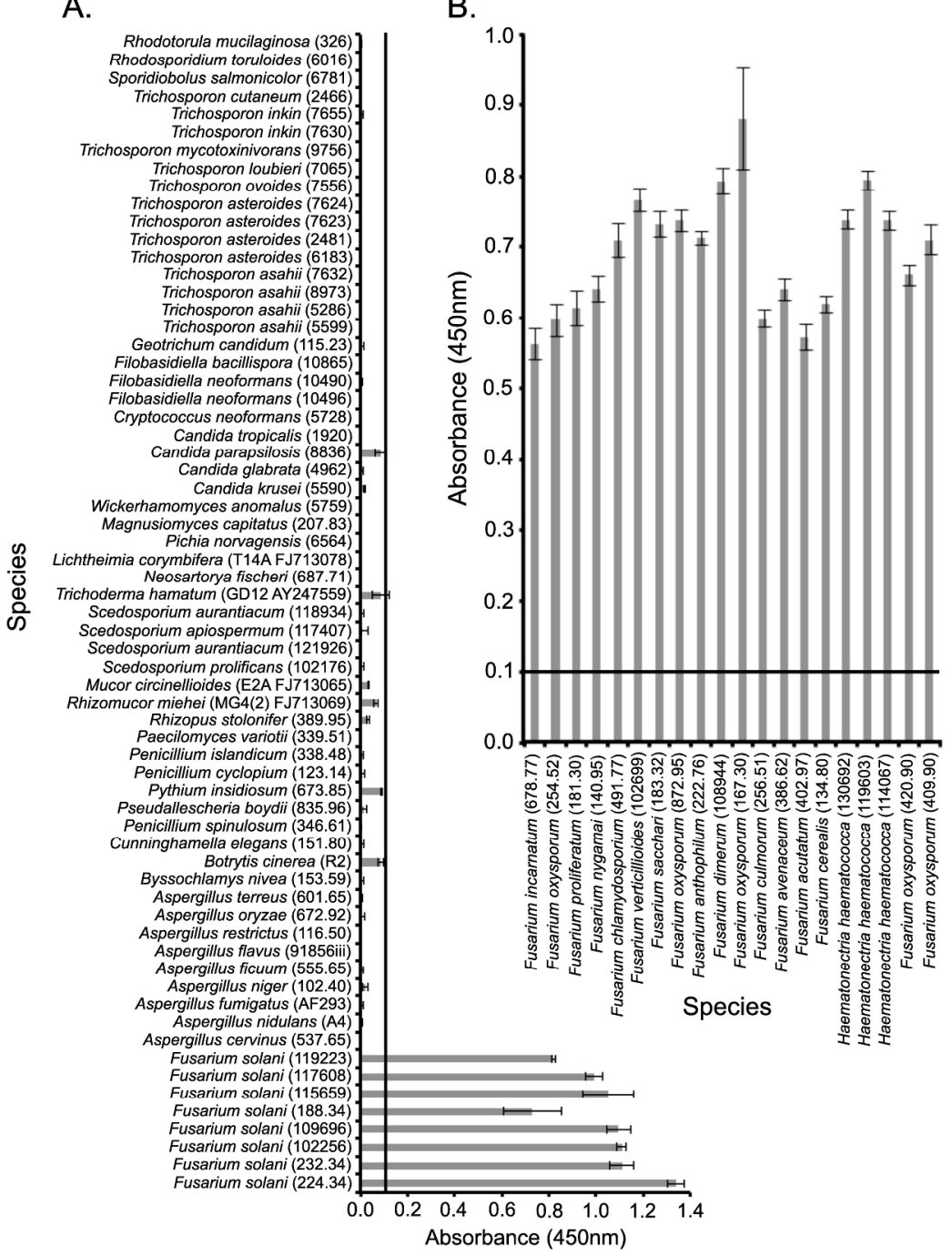

Figure 1. Al-Maqtoofi \& Thornton

Figure 1. Specificity of ED7 determined by Enzyme-Linked Immunosorbent Assay tests of surface washings containing water-soluble antigens from Fusarium species and related and unrelated yeasts and molds. (A) ELISA absorbance values at $450 \mathrm{~nm}$ for antigens from F. solani and unrelated yeasts and molds and (B) for antigens from the F. solani teleomorph Haematonectria haematococca and related Fusarium species. Wells were coated with $60 \mu \mathrm{g}$ protein $\mathrm{ml}-1$ buffer. Bars are the means of three biological replicates \pm standard errors and the threshold absorbance value for detection of antigen in ELISA is $\geq 0.100$ (indicated by lines on graphs). Numbers in parentheses after species names denote strain numbers with further details of strains provided in Table S1.

$279 \times 361 \mathrm{~mm}(300 \times 300 \mathrm{DPI})$ 

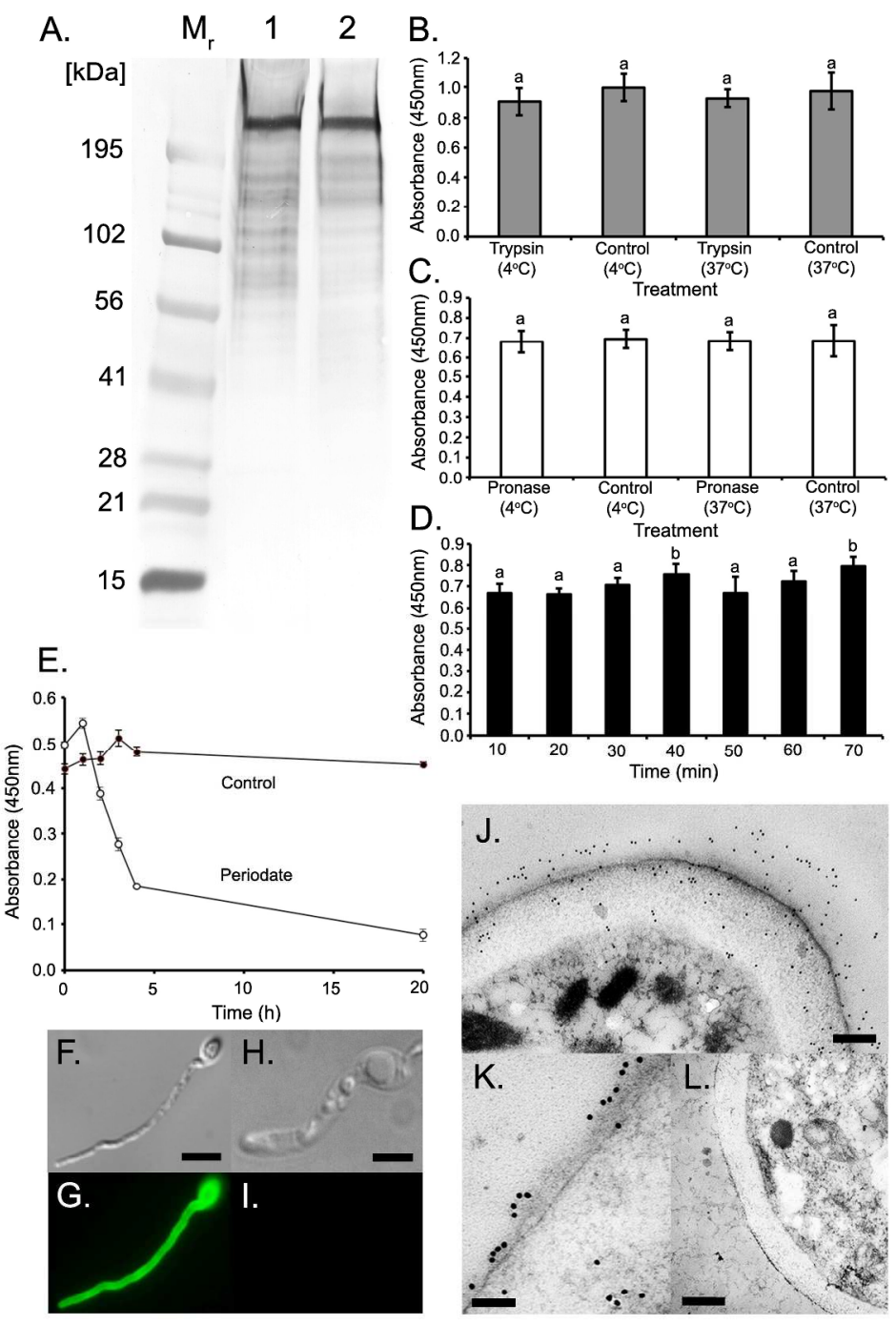

Figure 2. Al-Maqtoofi \& Thornton

Figure 2. Characterisation of the ED7 antigen and its epitope and spatial distribution of the antigen in spores and hyphae. (A) Western immunoblot with ED7 using culture fluid from 2-d-old PDB cultures of F. solani CBS224.34 (lane 1) and F. oxysporum f.sp. lycopersici CBS167.30 (lane 2). Wells were loaded with $1.6 \mu \mathrm{g}$ of protein. Mr denotes molecular weight in $\mathrm{kDa}$. Note the major extracellular antigen with molecular weight of $200 \mathrm{kDa}$. (B) Absorbance values from ELISA tests with ED7 using surface washings containing watersoluble antigens immobilized to the wells of microtitre plates and treated with trypsin or PBS only (control) at $40 \mathrm{C}$ and $37 \mathrm{oC}$. Bars are the means of three biological replicates \pm standard errors and bars with the same letter are not significantly different at $p<0.001$ (ANOVA and Tukey-Kramer test). (C) Absorbance values from ELISA tests with ED7 using surface washings containing water-soluble antigens immobilized to the wells of microtitre plates and treated with pronase or Milli-Q H2O only (control) at $40 \mathrm{OC}$ and $370 \mathrm{oC}$. Bars are the means of three biological replicates \pm standard errors and bars with the same letter are not significantly different at $p<0.001$ (ANOVA and Tukey-Kramer test). (D) Stability of the water-soluble ED7 antigen following heating of surface washings at $1000 \mathrm{C}$ over a 70 min period. Treated antigen was subsequently 
immobilized to the wells of microtitre plates and assayed by ELISA. Bars are the means of three biological replicates \pm standard errors and bars with the same letter are not significantly different at $p<0.001$ (ANOVA and Tukey-Kramer test). (E) Absorbance values from ELISA tests with ED7 using surface washings containing water-soluble antigens immobilized to the wells of microtitre plates and treated with periodate (open circles) or with acetate only control (closed circles) at $40 \mathrm{C}$ over a $20 \mathrm{~h}$ period. Each point is the mean of three biological replicates \pm standard errors. (F to I) Photomicrographs of F. solani CBS224.34 immunostained with ED7 or TCM control and goat anti-mouse polyvalent Ig fluorescein isothiocyanate (FITC) conjugate. (F) Brightfield image of germinated conidium with hypha probed with ED7 followed by fluorochrome conjugate (scale bar $=6 \mu \mathrm{m}$ ); $(\mathrm{G})$ Same field of view as panel $F$ but examined under epifluorescence. Note intense staining of the cell wall of microconidium and hypha; $(\mathrm{H})$ Brightfield image of germinated conidium with hypha probed with TCM (negative control) followed by FITC conjugate (scale bar

$=3 \mu \mathrm{m}$ ); (I) Same field of view as panel $\mathrm{H}$ but examined under epifluorescence. Note lack of staining, demonstrating specific binding of ED7 to surface antigen. (J-L) Immunogold labeling of sections of conidia and hyphae of F. solani CBS224.34. (J) Transverse section of conidium incubated with ED7 and anti-mouse immunoglobulin $20 \mathrm{~nm}$ gold particles, showing antigen in the cell wall and in an extracellular fimbrial matrix surrounding the spore (scale bar $=250 \mathrm{~nm}$ ); $(\mathrm{K})$ Longitudinal section of hypha incubated with ED7 and antimouse immunoglobulin $20 \mathrm{~nm} \mathrm{~g}$ $279 \times 361 \mathrm{~mm}(300 \times 300 \mathrm{DPI})$ 